\title{
Interwell Connectivity and Diagnosis Using Correlation of Production and Injection Rate Data in Hydrocarbon Production
}

\author{
ANNUAL REPORT \\ Reporting Period Start Date: June 2005 \\ Reporting Period End Date: May 2006 \\ Principal Authors \\ Dr. Jerry L. Jensen, TAMU \\ Dr. Larry W. Lake, UT-Austin \\ Ali Al-Yousef, UT-Austin \\ Dan Weber, UT-Austin \\ Ximing Liang, UT-Austin \\ Nazli Demiroren, TAMU \\ Danial Kaviani, TAMU
}

August, 2006

DOE Contract No. DE-FC26-03NT15397

Submitting Organization:

Texas Engineering Experiment Station

Texas A\&M University, College Station, TX 77843-3116

Subcontractor: Larry W. Lake

Petroleum and Geosystems Engineering

University of Texas, Austin, TX 78712 


\section{DISCLAIMER}

"This report was prepared as an account of work sponsored by an agency of the United States Government. Neither the United States Government nor any agency thereof, nor any of their employees, makes any warranty, express or implied, or assumes any legal liability or responsibility for the accuracy, completeness or usefulness of any information, apparatus, product, or process disclosed, or represents that its use would not infringe privately owned rights. Reference herein to any specific commercial product, process, or service by trade name, trademark manufacturer, or otherwise does not necessarily constitute or imply its endorsement, recommendation, or favoring by the United States Government or any agency thereof. The views and opinions of authors expressed herein do not necessarily state or reflect those of the United States Government or any agency thereof." 


\begin{abstract}
This report details progress on inferring interwell communication from well rate fluctuations. Starting with the procedure of Albertoni and Lake (2003) as a foundation, the goal of the project is to develop further procedures to infer reservoir properties through weights derived from correlations between injection and production rates. A modified method, described in Jensen et al. (2005) and Yousef et al. (2005), and herein referred to as the "capacitance model", produces two quantities, $\lambda$ and $\tau$, for each injector-producer well pair.
\end{abstract}

We have focused on the following items:

1. Approaches to integrate $\lambda$ and $\tau$ to improve connectivity evaluations. Interpretations have been developed using Lorenz-style and log-log plots to assess heterogeneity. Testing shows the interpretations can identify whether interwell connectivity is controlled by flow through fractures, high-permeability layers, or due to partial completion of wells. Applications to the South Wasson and North Buck Draw Fields show promising results.

2. Optimization of waterflood injection rates using the capacitance model and a power law relationship for watercut to maximize economic return. Initial tests using simulated data and a range of oil prices show the approach is working.

3. Spectral analysis of injection and production data to estimate interwell connectivity and to assess the effects of near-wellbore gas on the results. Development of methods and analysis are ongoing.

4. Investigation of methods to increase the robustness of the capacitance method. These methods include revising the solution method to simultaneously estimate $\lambda$ and $\tau$ for each well pair. This approach allows for further constraints to be imposed during the computation, such as limiting $\tau$ to a range of values defined by the sampling interval and duration of the field data. This work is proceeding.

Further work on this project includes the following.

1. Refinement and testing of the waterflood optimization process, including optimization on more complex situations e.g., time effects on revenue and water injection and disposal costs.

2. Completion of the spectral-based analysis and determination of the effects of nearwellbore gas on the results.

3. Revision of the capacitance model procedures to provide more robust results which are insensitive to the initial estimates of $\tau$ needed in the nonlinear regression. 


\section{TABLE OF CONTENTS}

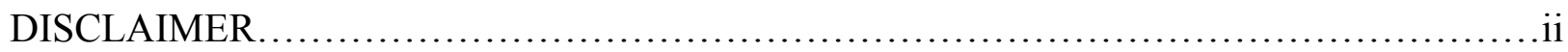

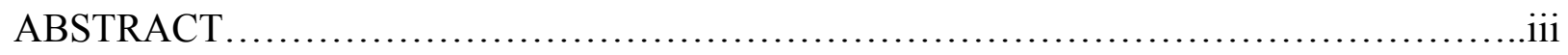

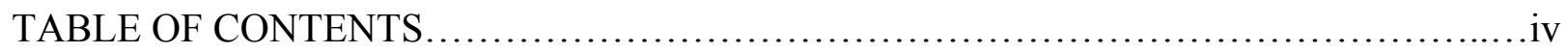

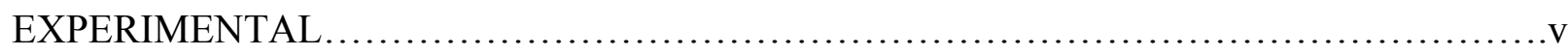

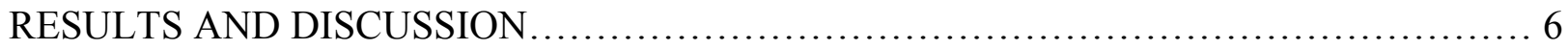

PART 1. Further interpretation of capacitance model parameters ............... 6

PART 2. Waterflood optimization using capacitance model results .............. 35

PART 3. Spectral analysis of injection and production data $\ldots \ldots \ldots \ldots \ldots \ldots \ldots . \ldots . \ldots . \ldots 3$

PART 4. Further investigation of capacitance model characteristics .............. 72

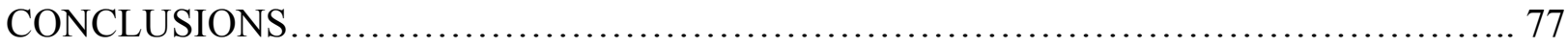

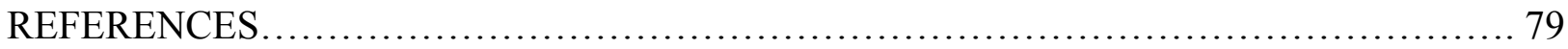




\section{EXPERIMENTAL}

No experimental procedures were involved in this project. 


\section{RESULTS AND DISCUSSION \\ PART 1. FURTHER INTERPRETATION OF CAPACITANCE MODEL PARAMETERS}

In the capacitance model, two coefficients are determined for each injector-producer pair; one parameter, $\lambda$, quantifies the connectivity and another, $\tau$, quantifies the fluid storage in the vicinity of the pairs. This section describes the development of this method into a diagnostic tool to enhance inference about preferential transmissibility trends and the presence of flow barriers.

\subsection{Introduction}

Most real reservoirs, if not all, undergoing a waterflood are heterogeneous where different geological conditions usually exist in the vicinity of injector-producer pairs. Different methods have been used to quantitatively determine the interwell connectivity and map reservoir heterogeneity from comparing the rate performance of a producing well with that of the surrounding injectors. Albertoni and Lake (2003) estimated interwell connectivity based on a linear model with coefficients estimated by multiple linear regression. The linear model coefficients quantitatively indicate the communication between a producer and the injectors in a waterflood. Yousef et al. (2005) used a more complete model that includes capacitance (compressibility) as well as resistive (transmissibility) effects. For each injector-producer pair, two coefficients are determined; one parameter (the weight) quantifies the connectivity, and another (the time constant) quantifies the degree of fluid storage between the wells. All the synthetic field applications exhibited in Yousef et al (2005) were homogeneous; the emphasis was on validation of the new aspects that the capacitance model has over the method proposed by Albertoni and Lake (2003).

The capacitance model (CM) incorporates the effects of primary production, multiple injectors, and bottom hole pressure (BHP) change for multiple producers, or (Yousef et al, 2005):

$$
\begin{aligned}
\hat{\mathrm{q}}_{\mathrm{j}}(\mathrm{n})= & \lambda_{\mathrm{p}} \mathrm{q}\left(\mathrm{n}_{0}\right) \mathrm{e}^{\frac{-\left(\mathrm{n}-\mathrm{n}_{0}\right)}{\tau_{\mathrm{p}}}}+\sum_{\mathrm{i}=1}^{\mathrm{i}=\mathrm{I}} \lambda_{\mathrm{ij}} \mathrm{j}_{\mathrm{ij}}^{\prime}(\mathrm{n}) \\
& +\sum_{\mathrm{k}=1}^{\mathrm{k}=\mathrm{K}} v_{\mathrm{kj}}\left[\begin{array}{l}
\mathrm{p}_{\mathrm{wf}_{\mathrm{kj}}}\left(\mathrm{n}_{0}\right) \mathrm{e}^{\frac{-\left(\mathrm{n}-\mathrm{n}_{0}\right)}{\tau_{\mathrm{kj}}}} \\
-\mathrm{p}_{\mathrm{wf}_{\mathrm{kj}}}(\mathrm{n})+\mathrm{p}_{\mathrm{wf}_{\mathrm{kj}}}^{\prime}(\mathrm{n})
\end{array}\right] \ldots \ldots \ldots \ldots \ldots \ldots \ldots \ldots \ldots \ldots \ldots \ldots \ldots
\end{aligned}
$$

where,

$$
\begin{aligned}
& \mathrm{i}_{\mathrm{ij}}^{\prime}(\mathrm{n})=\sum_{\mathrm{m}=\mathrm{n}_{0}}^{\mathrm{m}=\mathrm{n}} \frac{\Delta \mathrm{n}}{\tau_{\mathrm{ij}}} \mathrm{e}^{\frac{(\mathrm{m}-\mathrm{n})}{\tau_{\mathrm{ij}}}} \mathrm{i}_{\mathrm{ij}}(\mathrm{m}) \\
& \mathrm{p}_{\mathrm{wf}_{\mathrm{kj}}}^{\prime}(\mathrm{n})=\sum_{\mathrm{m}=\mathrm{n}_{0}}^{\mathrm{m}=\mathrm{n}} \frac{\Delta \mathrm{n}}{\tau_{\mathrm{kj}}} \mathrm{e}^{\frac{(\mathrm{m}-\mathrm{n})}{\tau_{\mathrm{kj}}}} \mathrm{p}_{\mathrm{wf}_{\mathrm{kj}}}(\mathrm{m})
\end{aligned}
$$

$\lambda_{\mathrm{p}}$ and $\tau_{\mathrm{p}}$ are the weighting factor and time constant for the primary production contribution to the estimated rate $\hat{\mathrm{q}}_{\mathrm{j}}$ of producer $\mathrm{j}$. $\lambda_{\mathrm{ij}}$ is the weight between injector $\mathrm{i}$ and producer $\mathrm{j}$ that indicates the connectivity between the (ij) well pair; $\tau_{\mathrm{ij}}$ is the time constant for the medium between injector $i$ and producer $j ; i_{i j}^{\prime}(n)$ is the convolved or filtered injection rate at step $n$ and $p^{\prime}{ }_{w f k j}(n)$ is 
the convolved BHP at step $\mathrm{n}$ for producer $\mathrm{k}$; $v_{\mathrm{kj}}$ is a coefficient that determines the effect of changing the BHP of producer $\mathrm{k}$ on the production rate of producer $\mathrm{j}$. The entire last term disappears if all K of the producer BHP's are constant (Yousef et al, 2005).

In our previous work, two versions of the capacitance model were introduced: the balanced capacitance model (BCM), and the unbalanced capacitance model (UCM). A waterflood is balanced when the field-wide injection rate is approximately equal to field-wide production rate. If this is the case, the BCM (Eq. 1) should be used. However, if the waterflood is unbalanced, a constant term $\left(\mathrm{q}_{\mathrm{oj}}\right)$ should be added to Eq. 1, which forms the UCM.

In both versions, the model parameters $\left(\lambda_{\mathrm{ij}}\right.$, and $\left.\tau_{\mathrm{ij}}\right)$ are the ones targeted in this work where they are determined by using the injection and production data through an optimization procedure (Yousef, 2006).

This paper exhibits new applications of the CM to synthetic fields with complex geological features. Since a producer usually communicates with multiple injectors, the complex geological conditions are often not easily identified using the $\lambda$ and $\tau$ values individually. However, combining both sets of parameters in certain representations enhances the inference about the geological features. Two different representations are used: one representation is a log-log plot of the $\lambda$ 's against the $\tau$ 's for a producer and nearby injectors, and another representation is the flow capacity plot where the $\lambda$ 's and the $\tau$ 's are combined using the idea of Lorenz plots. Both of representations enhance the inference about specific geological features in the vicinity of a producer.

The synthetic field and real field applications show that the relation between $\lambda$ 's and corresponding $\tau$ 's are consistent with the known heterogeneity, the distance between wells, and their relative positions. The flow capacity plots and the log-log plots are capable of identifying whether the connectivity of an injector-producer well pair is through fractures, a highpermeability layer, multiple-layers or through partially completed wells.

\subsection{Procedure}

Two different representations are proposed to enhance the inference about reservoir heterogeneity using the estimated parameters from the CM: the flow capacity plot, and the log-log plot of $\lambda$ 's against the $\tau$ 's. The mathematical developments of both representations are addressed in this section.

\subsubsection{The flow capacity plot}

The idea of flow versus storage was developed initially in the petroleum literature to estimate injection sweep efficiency in layered reservoir. This method relates the relative flow of a given layer to its associated pore volume, usually in a flow-storage diagram (Lorenz plots or flow capacity plots). These plots can be used quantitatively to describe reservoir geology. For example, $50 \%$ of flow comes from only $10 \%$ of the pore volume of a reservoir or a layer, which indicates fast flow paths.

The flow capacity plots estimated from the model parameters are different from the conventional Lorenz plots (Schmalz and Rahme, 1950). The Lorenz plots are based on permeability and sometimes porosity data obtained from measured samples (usually core plugs) taken from the reservoir where the spatial relationships of the samples are ignored, while the flow capacity plots are based on inferred parameters ( $\lambda$ 's and $\tau$ 's) from dynamic data (injection and production) in which these parameters account for all variations in reservoir properties in the vicinity of a producer. Shook (2003) also developed such plots (flow-storage diagram) based on 
estimated results from tracer tests. Therefore, the flow capacity plots, based on dynamic data, are likely to honor the flow paths and geological features in a reservoir.

The Lorenz plot, suggested by Schmalz and Rahme (1950), is used together with the estimated sets of $\lambda$ 's and $\tau$ 's between a producer and multiple injectors, to form the flow capacity plot. The Lorenz curve (Fig. 1) is a plot of cumulative flow capacity, $\mathrm{F}_{\mathrm{m}}$, versus cumulative thickness, $\mathrm{H}_{\mathrm{m}}$, where

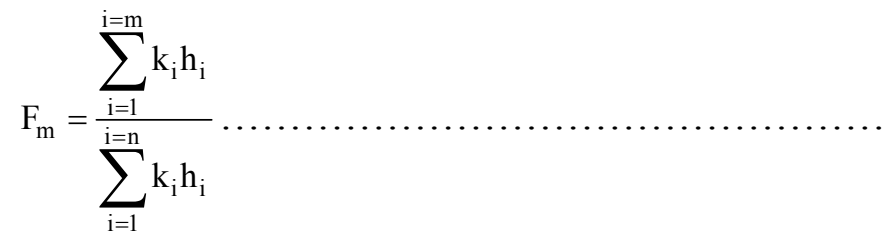

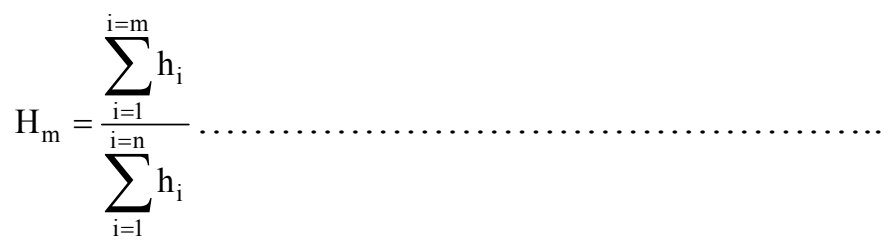

for a reservoir of $\mathrm{n}$ layers. The layers are arranged in order of decreasing permeability so that $\mathrm{m}$ $=1$ is the layer with thickness $h_{1}$ and the largest permeability $k_{1}$ whereas $m=n$ is the layer with thickness $h_{n}$ and the smallest permeability $k_{n}$. By definition, $0 \leq F_{m} \leq 1$ and $0 \leq H_{m} \leq 1$ for $1 \leq \mathrm{m}$ $\leq \mathrm{n}$. Because of the layer ordering, the Lorenz plot monotonically increases from $\mathrm{m}=1$ to $\mathrm{m}=\mathrm{n}$ with a monotonically decreasing slope. If the medium is homogeneous, all the permeability values are identical and the Lorenz plot is a straight line. Increasing levels of heterogeneity are indicated by movement of the Lorenz plot away from the straight line.

The Lorenz procedure can be modified by including porosity in the calculation (Craig, 1971; Lake, 1989). In place of the cumulative thickness, $\mathrm{H}_{\mathrm{m}}$, the cumulative storage capacity, $\mathrm{C}_{\mathrm{m}}$, is used, or:

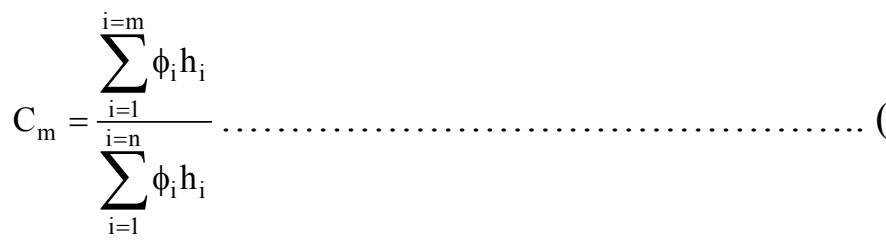

In this plot, a fraction of $\mathrm{F}_{\mathrm{m}}$ is provided by a fraction of $\mathrm{C}_{\mathrm{m}}$ of the reservoir. If porosity is constant, the Lorenz plot remains unchanged. The data must be arranged according to the ratio $\mathrm{k} / \phi$.

By analogy to the Lorenz plot, the flow capacity plot is formed using sets of $\lambda$ 's and $\tau$ 's for a producer and nearby injectors. This requires revisiting the physical definitions of the $\lambda$ and the $\tau$, introduced by Yousef et al (2005), to re-define the cumulative flow capacity $\left(\mathrm{F}_{\mathrm{m}}\right)$ and cumulative storage capacity $\left(\mathrm{C}_{\mathrm{m}}\right)$ in terms of model parameters.

The physical interpretation of the $\lambda$ of an injector-producer (ij) pair is given by: 


$$
\lambda_{\mathrm{ij}}=\frac{\mathrm{J}_{\mathrm{ij}}}{\sum_{\mathrm{j}}^{\mathrm{K}} \mathrm{J}_{\mathrm{ij}}} .
$$

where $\mathrm{J}$ is the partial productivity index and $\mathrm{K}$ is the total number of producers. This equation states that the ratio of the productivity between injector $\mathrm{i}$ and producer $\mathrm{j}$ to the sum of the productivity between the same injector and all producers is equal to the estimated weight between the (ij) well pair. Letting the dominator of Eq. 5 be constant, the $\lambda$ is equivalent to $\mathrm{k}_{\mathrm{ij}} \mathrm{h}_{\mathrm{ij}}$ between the (ij) well pair. In other words, the $\lambda$ quantifies the flow capacity between the (ij) well pair.

On another hand, the basic definition of the corresponding time constant is given by:

$$
\tau_{\mathrm{ij}}=\frac{\mathrm{c}_{\mathrm{tij}} \mathrm{V}_{\mathrm{p}_{\mathrm{ij}}}}{\mathrm{J}_{\mathrm{ij}}}
$$

where $c_{t}, V_{p}$, and $J$ are the total compressibility, the pore volume and the partial productivity between the (ij) well pair, respectively. Based on their definitions, the $\lambda$ and its corresponding $\tau$ are not totally independent because the $\lambda$ is directly proportional to $J$ and the $\tau$ is inversely proportional to the same $\mathrm{J}$.

Because we desire another parameter that reflects only the storage capacity between the injector-producer pair, the $\lambda$ multiplied by the corresponding $\tau$ provides this parameter, or:

$$
\lambda_{\mathrm{ij}} \tau_{\mathrm{ij}}=\frac{\mathrm{c}_{\mathrm{t}_{\mathrm{ij}}} \mathrm{V}_{\mathrm{p}_{\mathrm{ij}}}}{\sum_{\mathrm{j}}^{\mathrm{K}} \mathrm{J}_{\mathrm{ij}}}
$$

Similar to Eq. 5 by letting the dominator of Eq. 7 be constant, the $\lambda \times \tau$ quantifies the storage capacity between the well pair. Thus, the $\lambda$ and its corresponding $\lambda \times \tau$ are independent where the $\lambda$ quantifies the flow capacity and the $\lambda \times \tau$ quantifies the storage capacity for the medium between the same well pair.

Similar to Eqs. 2 and 4, the flow capacity curve is a plot of cumulative flow capacity $\left(\mathrm{F}_{\mathrm{m}}\right)$ against cumulative storage capacity $\left(\mathrm{C}_{\mathrm{m}}\right)$ where,

$$
F_{m}=\frac{\sum_{i=1}^{i=m} \lambda_{i j}}{\sum_{i=1}^{i=1} \lambda_{i j}} \ldots
$$

and

$$
\mathrm{C}_{\mathrm{m}}=\frac{\sum_{\mathrm{i}=1}^{\mathrm{i}=\mathrm{m}} \lambda_{\mathrm{ij}} \tau_{\mathrm{ij}}}{\sum_{\mathrm{i}=1}^{\mathrm{i}=1} \lambda_{\mathrm{ij}} \tau_{\mathrm{ij}}}
$$


for producer $\mathrm{j}$ with I injectors. The data are arranged in order of decreasing $1 / \tau_{\mathrm{ij}}$ so that $\mathrm{i}=1$ is the injector-producer well pair with the smallest $\tau$ while $i=I$ is the injector-producer well pair with the largest $\tau$. Because of the data ordering, the flow capacity curve monotonically increases from $\mathrm{i}=1$ to $\mathrm{i}=\mathrm{I}$ with a monotonically decreasing slope.

The flow capacity plots can also be used to indicate specific geological features in the vicinity of an injector. In this case, sets of $\lambda$ 's and $\tau$ 's for one injector and all producers are used to form the flow capacity plot.

The procedure, based on Lorenz plots, can make use of the extensive literature already available on the interpretation of such plots (Albertoni and Lake, 2003; Gunter et al, 1997; Cortez and Corbett, 2003).

\subsubsection{The log-log plot}

As discussed earlier, the $\lambda$ and the corresponding $\tau$ are inversely related through the productivity index J. For homogeneous reservoirs where each producer communicates with all injectors, a $\log -\log$ plot of $\lambda$ 's against $\tau$ 's for each producer with all injectors should give a straight line of slope -1. As described in our previous work, the $\log -\log$ plot of $\lambda$ 's against $\tau$ 's estimated from a homogenous synthetic field gives an approximate straight line of slope -1 . Compared to homogeneous reservoirs, the deviation of the $\lambda$ 's and the $\tau$ 's estimated from non-homogeneous reservoirs will indicate specific geological conditions in these reservoirs.

\subsection{Results}

The technique was tested through its application to two synthetic fields and then to three real fields. The results of these applications are presented and discussed in this section.

\subsubsection{Application to synthetic fields}

This section provides new applications of the CM to synthetic fields with different geological features. The emphasis will be on the consistency of the estimated model parameters ( $\lambda$ 's and $\tau$ 's) with the imposed geology, and the capability of the flow capacity plots to indicate the geological conditions imposed in each case.

The BCM approach was applied to the same numerically simulated synthetic fields (Synfields) exhibited in Yousef et al (2005): one of 5 injectors and 4 producers (the 5x4 Synfield) and a second of 25 injectors and 16 producers (the 25x16 Synfield). The 5x4 Synfield consists of five layers while the $25 \times 16$ Synfield consists of three layers. They both are flowing undersaturated oil. The injector-producer distance is $800 \mathrm{ft}$ for the $5 \times 4$ Synfield and $890 \mathrm{ft}$ for the $25 \times 16$ Synfield. The oil, water, and rock compressibility are 5x10-6, 1x10-6, and 1x10-6 psi-1, respectively. The oil-water mobility is equal to one. Both synfields have only vertical wells. All producers BHP's are equal. The characteristics of the synfields are similar to those of the real case to which the techniques will be applied later.

$5 \times 4$ Synfield. Several cases with different geological conditions were analyzed for this field. We used the same injection data provided in our previous work. These data were randomly selected from different wells in a real field and proportionally modified to be in agreement with the Synfield injectivity. The numerical simulation extends to $\mathrm{n}=100$ months, with $\Delta \mathrm{n}=1$ month.

Homogeneous reservoir. The first and simplest case is a homogenous reservoir with an isotropic permeability of $40 \mathrm{md}$. Because the total production rate is equal to the total injection rate, the BCM approach is applied. This case was demonstrated and extensively discussed in 
Yousef et al (2005) where the $\lambda$ 's and the $\tau$ 's are estimated and represented. Tables 1 and 2 list the numerical values of the $\lambda$ 's and the $\tau$ 's, respectively.

As mentioned earlier, the $\lambda$ and the corresponding $\tau$ are inversely related through the productivity index component $(\mathrm{J})$. The $\log -\log$ plot of $\lambda$ 's against $\tau$ 's estimated from the $5 \times 4$ homogenous Synfield gives an approximate straight line of slope -1 (Fig. 2). All the data lump in one single group, which indicates that all injectors communicate with producers through layers having the same properties.

The flow capacity plots are developed using sets of $\lambda$ 's and $\tau$ 's for each producer and all injectors (Fig. 3). All flow capacity plots are near straight lines, which indicate that the Synfield is homogeneous. The straight line suggests that the fraction of the total flow capacity observed in a producer is equal to the fraction of the total storage capacity swept by injectors. This is a typical behavior of homogeneous, isotropic field. Similar to the flow capacity plot, the conventional Lorenz plot is a straight line when the reservoir is homogeneous where all the permeability values are identical.

High permeability layer. As commented earlier, the $5 \times 4$ Synfield consists of five layers. In this case, a set of injectors is completed in large permeability layers, and another set is completed in small permeability layers. The permeability of layer 5 (L5) is large and equal to $500 \mathrm{md}$; the permeabilities of other layers (L1-L4) are relatively small and equal to $10 \mathrm{md}$. The vertical permeability is $0.1 \mathrm{md}$. Injectors I01, I02, and I03 are completed in L1-L4, while injectors I04 and $\mathrm{I} 05$ are only completed in the L5. All producers are completed in all layers. Figure 4 shows the layers, the permeability, and the completions of all wells. All other parameters are similar to the $5 \times 4$ homogeneous Synfield (the base case).

The BCM was applied and the $\lambda$ 's and $\tau$ 's were estimated. The match to the total production rate yields $\mathrm{R}^{2}=0.997$. In Fig. 5 , the $\lambda_{\mathrm{ij}}$ are represented by arrows or cones that start from injector $i$ and point to producer $j$. The larger the arrow, the larger the value of the weight and the greater connectivity between the two wells. Similarly, Fig. 6 shows the same representation for $\tau_{\mathrm{ij}}$ which the larger the arrow, the larger the value of the time constant and dissipation between the two wells.

The estimated $\lambda$ 's are the same as the base case results (Fig. 7) in which the $\lambda$ 's do not carry information about the high permeability layer (L5) or about the well completions. Because each reservoir layer is homogeneous and all producers are completed in all layers, the $\lambda$ 's estimated in this case, based on their basic definition (Eq. 5) are independent from reservoir permeability and they are only function of distance between wells and their relative locations. This explains that the estimated $\lambda$ 's in this application are the same as the base case results. However, the estimated $\tau$ 's of I04 and I05 are much smaller than that of I01, I02, and I03 (Fig. 6). This is consistent with the completions of injection wells where I04 and I05 are completed in the high permeability layer (L5), and other injectors are completed in the low permeability layers (L1-L4). Therefore, the $\tau$ 's give more insight about the geological conditions and completions imposed in this case than the $\lambda$ 's.

The inconsistency between the both sets of parameters in describing the geological conditions imposed in this case makes the inference about geological conditions from the $\lambda$ and $\tau$ values separately not easy. Thus, combining both sets of parameters in certain representations (e.g. the $\log -\log$ plots, the flow capacity plots) could enhance the inference about the geological features.

Unlike the base case, the $\log -\log$ plot of $\lambda$ 's against $\tau$ 's estimated in this case naturally separate into two different groups where each group gives an approximate straight line of slope -1 (Fig. 8). One group consists of parameters estimated with I04 and I05, and a second group includes parameters estimated with I01, I02, and I03. Since the estimated $\lambda$ 's are the same as the base 
case results and the group of parameters associated with I04 and I05 is characterized by smaller $\tau$ 's than the group with other injectors, we conclude that I04 and I05 communicate with all producers through large permeability layers, while other injectors communicate through small permeability layers. This is consistent with the geological conditions imposed in this case. Therefore, the $\log -\log$ plot of $\lambda$ 's against $\tau$ 's enhance the inference about certain geological conditions than can not be easily identified using the $\lambda$ and $\tau$ values separately.

Another representation to combine both sets of parameters is the flow capacity plot. The flow capacity plots are developed using sets of $\lambda$ 's and $\tau$ 's for each producer and all injectors (Fig. 9). All flow capacity curves (dynamic F-C curves) are not straight lines, which indicate that the Synfield is not homogeneous. For each producer, the flow capacity plot indicates two distinct geological layers, as depicted by the two straight lines (Fig. 9). The first straight line with a large slope consists of I04 and I05, while the small slope straight line consists of I01, I02, and I03. Because the estimated $\lambda$ 's are the same as the base case results, we conclude that I04 and I05 communicate with all producers through a high permeability layer while other injectors communicate with all producers through a small permeability layer. This is consistent with the geological conditions imposed in this case. Therefore, the flow capacity plots constructed from the $\lambda$ 's as well as $\tau$ 's reveal certain geological features that can not be easily identified using the $\lambda$ and $\tau$ values separately.

The static flow capacity curve estimated using permeability and porosity values of each grid block is depicted in Fig. 9. Similar to the dynamic F-C curves, the static F-C curve indicates two distinct geological conditions; however, the deviation shown by the static F-C curve from the homogeneous behavior is larger than that depicted by the dynamic F-C. The Lorenz coefficient (Schmalz and Rahme, 1950) $\left(\mathrm{L}_{\mathrm{c}}\right)$ is a measure of movement or deviation of the $\mathrm{F}-\mathrm{C}$ curve from the $45^{\circ}$ line. If the medium is homogeneous, the flow capacity plot is the $45^{\circ}$ line and consequently $\mathrm{L}_{\mathrm{c}}=0$. Increasing the level of heterogeneity are indicated by movement of the flow capacity plot away from the $45^{\circ}$ line with $\mathrm{L}_{\mathrm{c}}$ increasing but always less than unity. For this case, the $\mathrm{L}_{\mathrm{c}}$ for the static F-C curve is 0.7 while the $\mathrm{L}_{\mathrm{c}}$ 's for the dynamic F-C curves of P01, P02, P03, and $\mathrm{P} 04$ are $0.2,0.3,0.3$, and 0.5 respectively.

Fractures. Two fractures both with one grid-size thickness are introduced in this case: one fracture between I01 and P01 with permeability of $1000 \mathrm{md}$, and a second between I03 and P04 with smaller permeability $(500 \mathrm{md})$. The rest of the Synfield is only $5 \mathrm{md}$. The two fractures extend to all layers and injectors and producers are completed in all encountered layers. All other parameters are similar to the base case.

The BCM was applied and the $\lambda$ 's and $\tau$ 's were estimated. The match to the total production rate yields $\mathrm{R}^{2}=0.997$. Figures 10 and 11 show the representations of the $\lambda$ 's and the $\tau$ 's.

The $\lambda$ 's between I01-P01 and I03-P04 are large, while the corresponding $\tau$ 's are very small. I01 and I03 mainly support P01 and P04, respectively (Fig. 10). This is consistent with the two fractures existing in this case. Also, the near injectors (I04 and I05) to P04 and I02 to P01 exhibit large $\lambda$ 's, while the corresponding $\tau$ 's are relatively large (Fig. 11). Therefore, both sets of parameters reflect the heterogeneity of the field.

The log-log plot of $\lambda$ 's against $\tau$ 's indicates three different groups (Fig. 12). Group 1, which includes the data for I01-P01 and I03-P04, reflects the two fractures existing in the field. Group 2 represents near well pairs with large $\lambda$ 's and large $\tau$ 's, and Group 3 shows distant well pairs with small $\lambda$ 's and large $\tau$ 's. The data are very scattered, which indicates a large deviation from homogenous reservoir. All of these observations are consistent with the imposed geology.

The flow capacity plots of P01 and P04 show a large deviation from the $45^{\circ}$ line (homogeneous reservoir) while the flow capacity plots of P02 and P03 do not reflect the 
heterogeneity of the field (Fig. 13). Because all injectors mainly support P01 and P04, the flow capacity plots of these producers will carry more insight about the imposed geology than that of other producers. This explains the small deviation of the flow capacity curves of P02 and P03 from the $45^{\circ}$ line (homogeneous reservoir).

The flow capacity plots of P01 and P04 indicate two distinct geological conditions in the vicinity of these producers. In both plots, two straight lines can be fitted to the flow capacity curves; the first is steeper than the second one. For each plot, the steep straight line suggests that a large fraction of the total flow capacity is provided by a very small fraction of the total pore volume swept by the injectors, which is usually an indication of existing fractures in the vicinity of the corresponding producer. Because P01 and P04 are supported by I01 and I03 through fractures, their flow capacity plots decisively indicate these fractures by the steep straight line. Also, the first straight line in the flow capacity of P01 is much steeper than that of P04, which is consistent with the difference in permeabilities of the fractures. The second straight line in both plots suggests that a small fraction of total flow capacity is provided by a large fraction of the total volume of the field; this usually contributes from injectors communicating through the matrix of the reservoir.

The static flow capacity curve estimated using permeability and porosity values of each grid block is depicted in Fig. 13. Similar to the dynamic F-C curves of P01 and P04, the static F-C curve indicates two distinct geological conditions; however, the deviation shown by the static F$\mathrm{C}$ curve from the homogeneous behavior is larger than that depicted by the dynamic F-C of the two producers. Thus, the $\mathrm{L}_{\mathrm{c}}$ for the static $\mathrm{F}-\mathrm{C}$ curve is 0.9 while the $\mathrm{L}_{\mathrm{c}}$ 's for the dynamic F-C curves of $\mathrm{P} 01$ and $\mathrm{P} 04$ are 0.68 , and 0.52 , respectively.

Compared to the high permeability layer case, the first straight lines in both plots of P01 and P04 are much steeper than that observed in the high permeability layer case (Fig. 9). This indicates that the flow capacity plots are capable of identifying whether the connectivity of an injector-producer well pair is through a fracture or a high-permeability layer.

Producer with partial completion. In this case, P04 is only completed in layer 5 (L5), while the other producers and injectors are completed in all layers (L1-L5). All reservoir layers are homogeneous and have the same permeability $(40 \mathrm{md})$. The vertical permeability is $0.4 \mathrm{md}$. All other parameters are similar to the base case.

The BCM was applied and the $\lambda$ 's and the $\tau$ 's were estimated. The partial completion of P04 can be easily inferred from the $\lambda$ 's representation (Fig. 14). The $\lambda$ 's of all injectors with P04 are very small, which are consistent with small productivity observed in P04 because of its completion. However, the estimated $\tau$ 's are the same as the base case results where the $\tau$ 's do not carry information about the partial completion of P04 (Fig. 15). Because the pore volume $\left(\mathrm{V}_{\mathrm{p}}\right)$ and the productivity $(\mathrm{J})$ in the basic definition of the $\tau$ (Eq. 6) are both function of the pay thickness of the corresponding producer, the $\tau$ does not depend on the pay thickness (Raghavan, 1991; Ibragimov et al., 2005). Therefore, the estimated $\tau$ 's in this case are expected not to reflect the partial completion of P04.

The log-log plot of $\lambda$ 's versus $\tau$ 's clearly indicates the partial completion of P04, represented by Group 2 (Fig. 16). Group 1 represents the parameters associated with other producers. However, all flow capacity plots do not indicate the partial completion of P04 (Fig. 17). This is attributed to the definitions of the cumulative flow capacity (Eq. 8) and the cumulative storage capacity (Eq. 9). Because the $\lambda$ 's are used in both equations and the geological conditions in this case are not reflected by the corresponding $\tau$ 's, the flow capacity plot for each producer usually shows a homogeneous reservoir flow capacity. Therefore, the flow capacity plot defined in this chapter is not able to indicate problems associated with completion of production wells. 
25x16 Synfield. Two cases are studied for this field: a homogeneous reservoir and reservoir with different sealing faults. We used a randomly generated injection data. The numerical simulation extends to $\mathrm{n}=415$ months, with $\Delta \mathrm{n}=1$ month (Yousef et al, 2005; Yousef, 2006).

Homogeneous reservoir. This case was extensively discussed in our previous work where the $\lambda$ 's and the $\tau$ 's are estimated and represented. The applications of the log-log plot and the flow capacity plot give the same results as that of the $4 \times 5$ homogeneous Synfield (Yousef, 2006).

Presence of sealing faults. In this case, four different sealing faults divide the entire reservoir into five isolated compartments. All layers are homogeneous and have the same permeability (40 $\mathrm{md})$. Wells are completed in all encountered layers. Other parameters are similar to the $25 \times 16$ homogeneous Synfield.

Figures 18 and 19 show the results for the BCM applied for this case. Results are just as expected. The presence of sealing faults can be easily inferred from the $\lambda$ 's and the $\tau$ 's representations. The values of $\lambda$ corresponding for well pairs located in different compartments are close to zero, while the corresponding values of $\tau$ for the same well pairs are very large. This shows no communication between these wells, which is consistent with the presence of the sealing faults.

The log-log plot of $\lambda$ 's versus $\tau$ 's indicates two different groups (Fig. 20). Group 1 reflects the values of $\lambda$ and $\tau$ for well pairs located in the same compartment. This group is characterized by relatively large $\lambda$ 's and small $\tau$ 's. Group 2 , characterized by very small $\lambda$ 's $(\lambda<0.03)$ and very large $\tau$ 's, includes the values of $\lambda$ and $\tau$ for well pairs located in different compartments. All of these observations are consistent with the presence of the sealing faults.

All flow capacity plots indicate two distinct geological conditions. The flow capacity plots for the four center producers (P06, P07, P10, P11) and the outer producers (P01, P04, P13, P16) are only shown in Figs. 21 and 22, respectively. The first straight line, parallel to the $45^{\circ}$ line, represents a homogeneous reservoir flow capacity. Because each compartment is homogeneous and the first straight line in each plot includes the model parameters for well pairs located in the same compartment, this straight line represents the fraction of total flow capacity provided by well pairs located in the same compartment. However, the straight line with flat behavior indicates that a fraction of the total storage capacity provides a negligible fraction of the total flow capacity. This is a typical aspect of presence of a reservoir seal. Therefore, all flat straight lines decisively indicate presence of the sealing faults in the vicinity of producers.

\subsubsection{Application to field data}

The technique was applied to three different sets of field data. The results of these applications are presented and discussed in this section. Unlike the Synfield applications we have no concrete standards (e.g., agreement with imposed geology) against which to compare results. Our truth test will be comparison against known geological features as much as possible.

The first real field application. The technique was applied to a portion (18 injectors and 13 producers) of the South Wasson Clearfork (SWCF) Field in West Texas. The reservoir is primarily dolomite and the average porosity and permeability are $6 \%$ and $4 \mathrm{md}$, respectively. Waterflooding began in 1980 where the initial waterflood pattern was inverted nine-spot, and then was converted to five-spot pattern in 1987. Fractures were observed in the SWCF injectors, which typically exist in carbonate reservoirs (Burbank, 1992). Since only a portion of the field was analyzed and the boundaries are open, the UCM approach is applied.

The data selected for the analysis was determined by examining the production, and injection rates and the producing gas oil ratio (GOR) of the SWCF field. The GOR was large when the waterflood started. By month 70 , the GOR was at a minimum and approximately equal to the 
solution gas-oil ratio, which indicates that the hydrocarbon in the reservoir is single-phase. Also, injection and production rates are in acceptable balance. However, the GOR fluctuated after month 137. Therefore, the analysis is carried out using the data starting in month 70 until month 137 which left 68 data points for the analysis. With this short injection and production data and 18 injectors, we know in advance that we will obtain suboptimal results and the estimated $\lambda$ 's will be imprecise. Moreover, the open boundaries introduce errors in the estimation of the $\lambda$ 's and some of the assumptions of the technique are not completely met. However, some general features can be inferred from the application of UCM model to the SWCF field data.

Figures 23 and 24 show representations of the positive $\lambda$ 's and the $\tau$ 's, respectively. The injection wells in the north and the west of the field have smaller $\lambda$ 's than those in the south-east. The interwell connectivity improves from the north-west to the south-east. Three injectors (7501, 7502, and 7505) mainly seem to have little influence on inner producers. Also, producers 7518, 7528, 7526, 7520 and 7540 appear to be unsupported. Injectors 7532, 7507, and 7506 predominantly exhibit much larger connectivity with producers in the south-east of the field than that of other injectors. With knowing that the field is very heterogeneous and also has very small average permeability ( $4 \mathrm{md}$ ), the estimated $\tau$ 's initiated from these injectors (Fig. 24) are relatively smaller than the ones observed in the synthetic field applications with permeability less than $5 \mathrm{md}$ (Yousef, 2006). All of these observations suggest that these injectors communicate with producers through high permeability layers, or most likely through fractures.

We investigated this issue by use the log-log plot of $\lambda$ 's versus $\tau$ 's. The log-log plot of $\lambda$ 's versus $\tau$ 's for all wells shows that the $\lambda$ and the corresponding $\tau$ are not inversely related, which is expected since the reservoir is not homogeneous (Fig. 25). Two groups can be determined according to the spread in $\tau$. Group 1, characterized by very small $\tau$, indicates that certain injectors communicate with producers through high permeability layers or through fractures while Group 2, described by large $\tau$, indicates that well pairs communicate through the matrix of the reservoir.

Figure 26 shows the flow capacity plots for producers supported by the three injectors (7532, 7507, and 7506). By comparing these flow capacity plots, especially for produces 7516 and 7519 , with the ones observed in synthetic case where one or more injector-producer pairs communicate through a fracture (see 5x4 Synfield with fractures), we are tempted to conclude that fractures exist in the vicinity of these two producers, or the key injectors $(7506,7507$, and 7532) communicate to producers 7516 and 7519 through fractures. However, the flow capacity plots of producers 7524,7521 , and 7513 do not reflect heterogeneity. This is attributed to the open boundaries effect. To validate any of these observations would require other data.

The fits to the production data are relatively good. For example, the $\mathrm{R}^{2}$ for producers 7516 and 7519 are 0.923 and 0.937 , respectively.

The second real field application. The UCM was applied as well to a portion (8 injectors and 12 producers) of the North Buck Draw (NBD) Field in Wyoming. The reservoir average porosity is $9.5 \%$, and the average permeability is $10.7 \mathrm{md}$. The reservoir fluid is near-volatile oil; fluid properties fall between those of black and volatile oils. The fluid meets the majority of volatileoil criteria, including large oil formation volume factors and solution gas-oil ratios. The bubblepoint pressure is 4680 psia, and the reservoir fluid is a single-phase, low-viscosity fluid above this pressure (Sellars and Hawkins, 1992).

The field commercial production began in June 1983. In 1988 a pressure maintenance project was initiated by injecting gas. Since the field is undergoing gas injection, the reservoir total compressibility is very large and is not constant, which violates one of the main assumptions of the technique, slightly compressible fluids. However, if the product of $c_{t} \Delta p$ is much smaller the 
one, the assumption of the slightly compressible fluids will be attained. In this situation, the technique will provide reasonable $\lambda$ 's while the estimated $\tau$ 's will indicate the dissipation associated with large compressibility.

The data selected for the analysis was determined by the same procedure used in the application of the UCM to SWCF field. However, the GOR increases with gas injection, as expected. The analysis is carried out using the data starting in month 35, which left 56 data points for the analysis. Because the reservoir total compressibility is large, the inference procedure most likely will not able to indicate the connectivity between distant injector-producer pairs. Therefore, the UCM approach is applied to producers and only their adjacent injectors.

Figure 27 shows the representation of the positive $\lambda$ 's. The injection wells in the west of the field have larger $\lambda$ 's than those in the east. Producers 33-6, 31-7, and 33-7 appear to be unsupported. The orientation of the $\lambda$ 's in wells 13-18, 11-18, and 12-7 indicate that the connectivity north-south direction is better than that of other directions.

Radioactive tracers were injected into the reservoir, and their occurrence was monitored at the producing wells from February 1989 until March 1993. Refunjol and Lake (1997) applied Spearman analysis to determined preferential flow trends in the NBD field and compared the results with injected tracer response. They related injection wells with their adjacent producers and used time lags to find an extreme coefficient $\left(r_{s}\right)$.

Because the Spearman correlation coefficient $\left(r_{s}\right)$ is another measure of the connectivity between injector-producer pair, a comparison between the $r_{s}$ 's and the $\lambda$ 's should provide a relation that is consistent with interwell connectivity between well pairs. When the two parameters are positive, the $r_{s}$ tends to be larger than the corresponding $\lambda$. Similarly, the $r_{s}$ is larger when both parameters are negative. Thus, the estimated $\lambda$ 's are more sensible than the $r_{s}$ 's in describing the connectivity between well pairs.

Figure 28 shows representations of the estimated $\tau$ 's, the Spearman time lags, and the tracer response breakthrough times. The orientation of the $\tau$ 's in wells 13-18, 11-18, and 12-7 suggest that the dissipation in the north-south direction is relatively smaller than that of other directions, which is in agreement with the orientation of the corresponding $\lambda$ 's in these wells (Fig. 27).

Because the tracer response breakthrough times are considered real field measurements, we use them as a base for validating the $\tau$ 's and the spearman time lags. For injector 23-8, the estimated $\tau$ 's are in agreement with the tracer response breakthrough times observed in the surrounding producers. For injector 11-18 and producer 33-7, Refunjol and Lake (1997) reported maximum $r_{\mathrm{s}}$ of 0.8 with 13 months time lag while the UCM estimates $\lambda$ of 0.1 and $\tau$ of 0.1 month for the same well pair. Because the tracer response was not observed in producer 33-7, we are tempted to conclude that the capacitance model results are more accurate than that of the Spearman analysis. Similar situation can be noticed for injector 31-7 and producer 23-8.

Figure 29 shows the flow capacity plots for some producers in the NBD field. The flow capacity plots for producers 33-18,33-7, and 31-18 indicate certain geological features in the surrounding area of each producer. These plots indicate two different geological features in which certain injectors communicate with the corresponding producer through fast flow paths or high permeability layers and the other injectors communicate through slow flow paths or low permeability layers. The flow capacity plots for the other producers (33-6, 33-13, and 13-7) do not carry information about the medium between these producers and the corresponding injectors. To validate any of these observations would require other data.

The fit to the production data are relatively good. For example, the $\mathrm{R}^{2}$ for producers $13-7$ and $33-12$ are 0.942 and 0.974 , respectively. 


\subsection{Discussion}

From applications of the CM exhibited in this work and others described in Yousef (2006), we can identify specific trends of the flow capacity curve according to the corresponding geological feature present in the surrounding area of a producer. To illustrate this point, Fig. 30 shows three curves of the flow capacity plot estimated from sets of $\lambda$ 's and $\tau$ 's. The first curve indicates a presence of fractures in the drainage volume of a producer represented by the steeper segment of the curve; the second indicates that certain injectors communicate with producer through high permeability layers and the other injectors communicate through low permeability layers. For the last curve, the flat behavior shows that a fraction of the total storage capacity or the total pore volume swept by injectors provides a negligible fraction of the total flow capacity. This is a typical aspect of nonpay zone or a reservoir seal.

However, there are cases where the flow capacity plots are not able to reflect the geological conditions present in the vicinity of the producer. As discussed earlier, the flow capacity plot can not identify the partial completion of a producer even though this can be easily inferred from the model parameters ( $\lambda$ 's and $\tau$ 's). As commented before, this is attributed to the definitions of the cumulative flow capacity (Eq. 8) and the cumulative storage capacity (Eq. 9) where both of them are function of the connectivity parameters $(\lambda)$. In this case, the flow capacity plot will indicate a homogeneous flow capacity.

On another hand, the log-log plot of $\lambda$ 's versus $\tau$ 's shows consistency with imposed geology in the application to synthetic fields where distinct groups corresponding to different geological conditions can be easily identified. In the application to real field data, the scattered in the data is large where distinct groups of data are not easily determined. The large scattered in the data is attributed to the typical heterogeneity of real fields.

Because the flow capacity plots are based on inferred parameters ( $\lambda$ 's and $\tau$ 's) from dynamic data (injection and production), different sources of error can render the inference about geological conditions. The possible sources of error are deviation from the assumptions on which the CM is based, correlation between injection rates and using short assessment interval, and the quality of injection and production rate measurements (Yousef et al., 2005; Yousef, 2006).

\subsection{Conclusions}

The capacitance model $\tau$ 's and $\lambda$ 's can be combined to enhance inference about preferential transmissibility trends and the presence of flow barriers. Complex geological conditions are often not easily identified using the $\lambda$ and $\tau$ values individually. However, combining both sets of parameters in certain representations enhances the inference about the geological features. Two different representations are used: one representation is the log-log plot of the $\lambda$ 's against the $\tau$ 's for a producer and nearby injectors; another representation is the flow capacity plot where the $\lambda$ 's and the $\tau$ 's are combined using the idea of Lorenz plots. Both of representations enhance the inference about specific geological features in the vicinity of a producer.

The synthetic field applications show that the relation between the $\lambda$ 's and the corresponding $\tau$ 's are consistent with known heterogeneity, the distance between wells, and their relative positions. The flow capacity plots and the log-log plots are capable of identifying whether the connectivity of an injector-producer well pair is through fractures, a high-permeability layer, or through partially completed wells.

The technique was first applied to data from the South Wasson Clear Fork Field in West Texas. Knowing that the field is very heterogeneous and also has very small permeability, the estimated time constants between the key injectors and certain producers indicate that these 
injectors communicate with producers through high permeability layers, or most likely through fractures. The flow capacity plots and the $\log -\log$ plot of $\lambda$ 's versus $\tau$ 's confirm the existing of fractures in the vicinity of some producers. The verification of these results would require other data; however, fractures typically exist in carbonate reservoirs.

Finally, the technique was applied to the North Buck Draw Field. Even though the field is undergoing gas injection, the estimated time constants indicate the dissipation associated with large compressibility. Compared to the tracer results, the capacitance model results are more accurate than that of the Spearman analysis. The flow capacity plots indicate that certain nearby injectors communicate with some producers through fast flow paths and other injectors communicate through low permeability layers. Verification of such results would require other data.

\subsection{Tables and Figures}

Table 1: The weights $\lambda_{i j}$ for $5 \times 4$ homogenous Synfield.

\begin{tabular}{|c|c|c|c|c|}
\hline & P01 & P02 & P03 & P04 \\
\hline I01 & 0.33 & 0.33 & 0.17 & 0.17 \\
\hline $\mathrm{I} 02$ & 0.33 & 0.17 & 0.33 & 0.17 \\
\hline $\mathrm{I} 03$ & 0.25 & 0.25 & 0.25 & 0.25 \\
\hline $\mathrm{I} 04$ & 0.17 & 0.33 & 0.17 & 0.33 \\
\hline $\mathrm{I} 05$ & 0.17 & 0.17 & 0.33 & 0.33 \\
\hline$\lambda_{\mathrm{p}}$ & 1.00 & 1.00 & 1.00 & 1.00 \\
\hline
\end{tabular}

Table 2: The time constants $\tau_{\mathrm{ij}}$ (month) for $\mathbf{5 \times 4}$ homogenous Synfield.

\begin{tabular}{|c|c|c|c|c|}
\hline & $P 01$ & $P 02$ & $P 03$ & $P 04$ \\
\hline$I 01$ & 0.60 & 0.59 & 0.81 & 0.80 \\
\hline 102 & 0.65 & 0.86 & 0.61 & 0.84 \\
\hline 103 & 0.66 & 0.62 & 0.63 & 0.62 \\
\hline 104 & 0.79 & 0.62 & 0.81 & 0.61 \\
\hline 105 & 0.81 & 0.81 & 0.62 & 0.62 \\
\hline$\tau_{p}$ & 0.00 & 0.00 & 0.00 & 0.00 \\
\hline
\end{tabular}




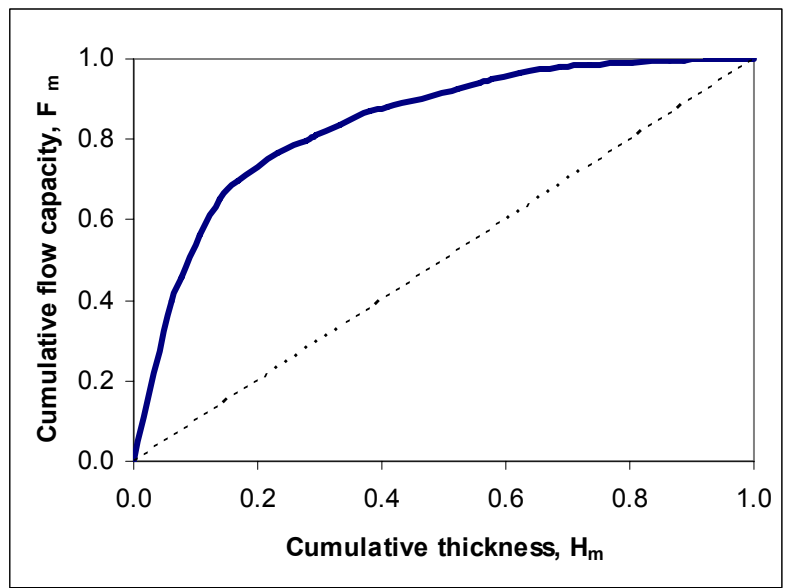

Figure 1: Cumulative flow capacity versus cumulative thickness. A schematic of the conventional Lorenz plot. The dashed line represents the $45^{\circ}$ line.

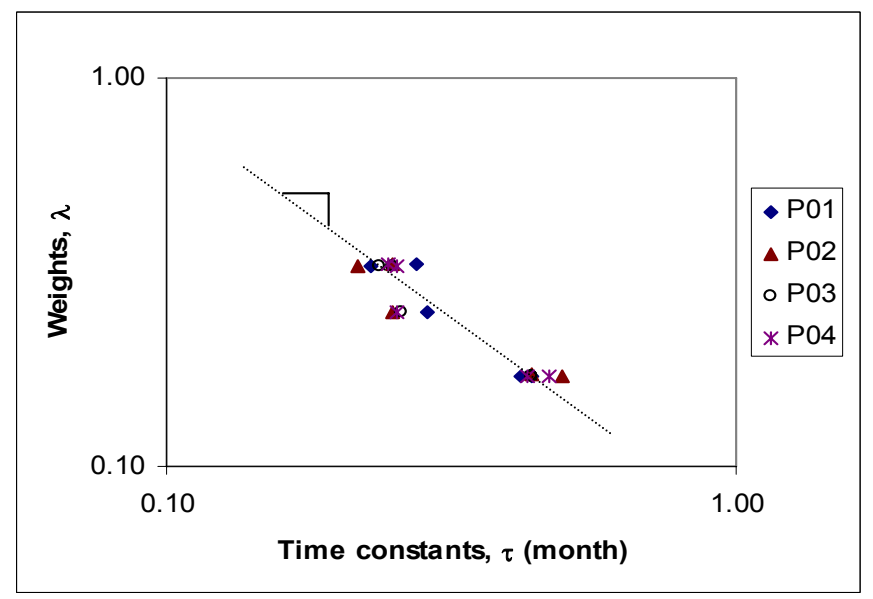

Figure 2: Log-log plot of $\lambda$ 's versus $\tau$ 's with a slope of approximately $-1.5 \times 4$ homogeneous Synfield. 

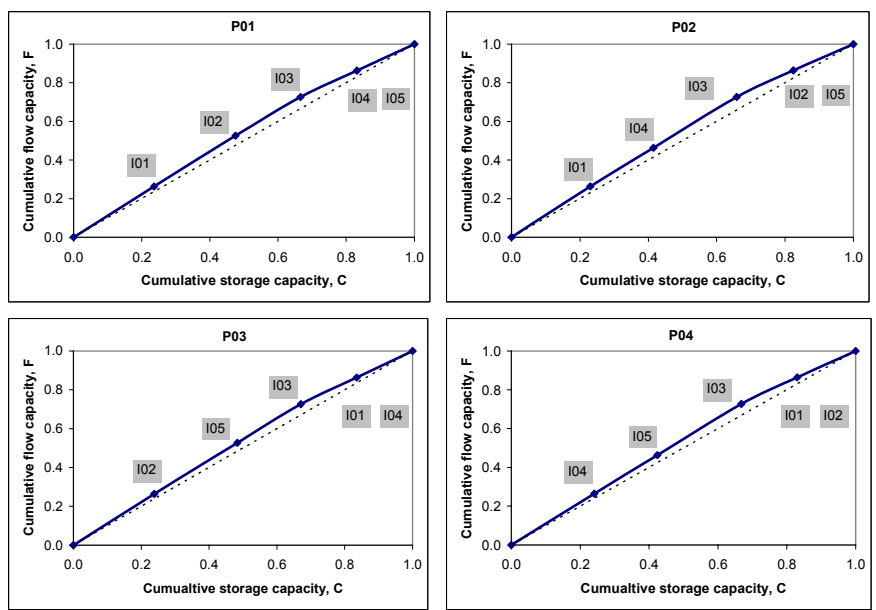

Figure 3: The flow capacity plots for all producers. The dashed line presents the $45^{\circ}$ line. 5x4 Synfield, homogeneous reservoir.

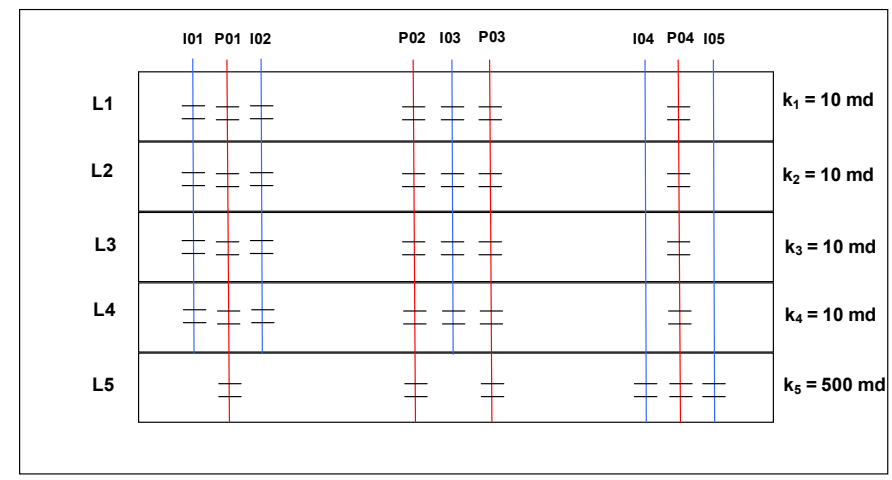

Figure 4: The layers, the permeabilities, and the well completions. 5x4 Synfield, high permeability layer.

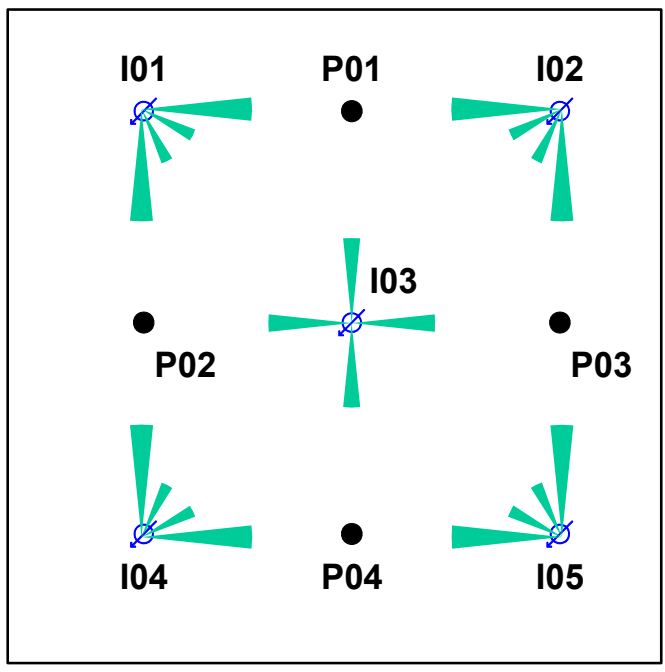

Figure 5: Representation of the weights $\lambda_{\mathrm{ij}}$. The length of the arrow is proportional to the value of the weight. $5 \times 4$ Synfield, high permeability layer. 


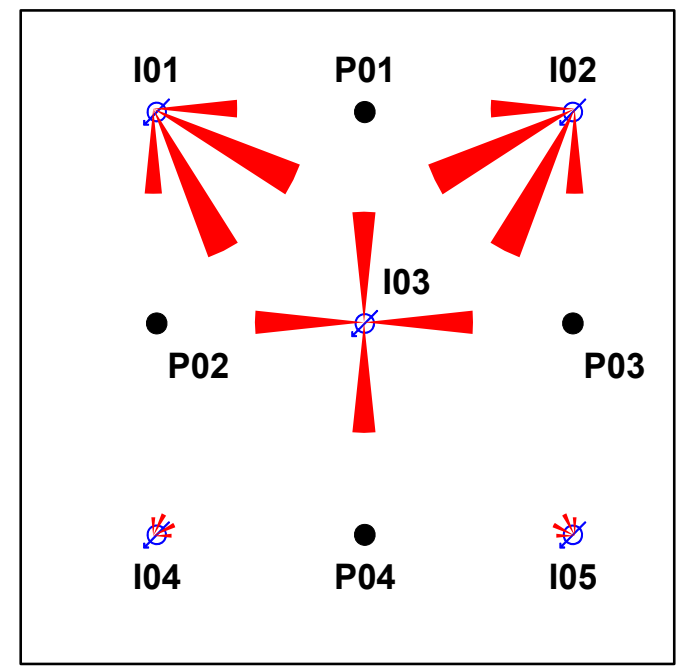

Figure 6: Representation of the time constants $\tau_{\mathrm{ij}}$ (month). The length of the arrow is proportional to the value of $\tau$. $5 \times 4$ Synfield, high permeability layer.

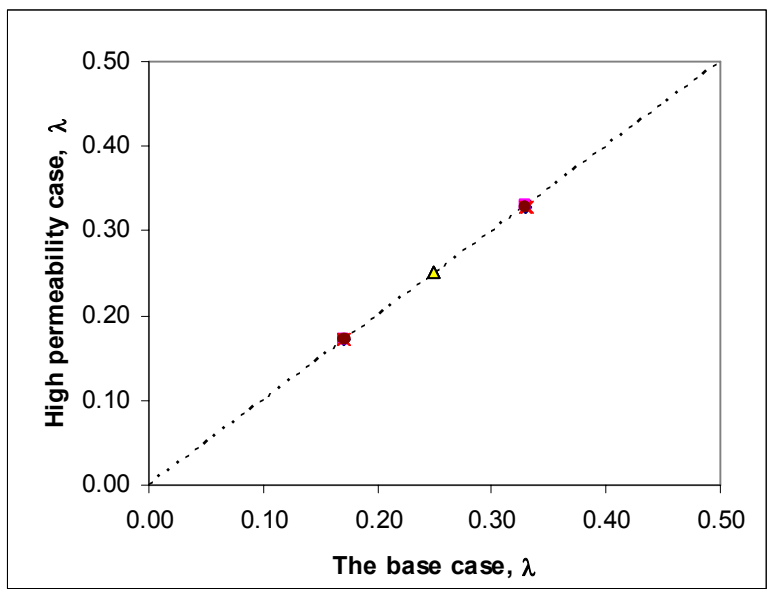

Figure 7: A crossplot of the $\lambda$ 's estimated from the high permeability layer case against the $\lambda$ 's estimated from the $5 \times 4$ homogeneous Synfield (the base case). The dashed line represents the $45^{\circ}$ line. $5 \times 4$ Synfield, high permeability layer. 


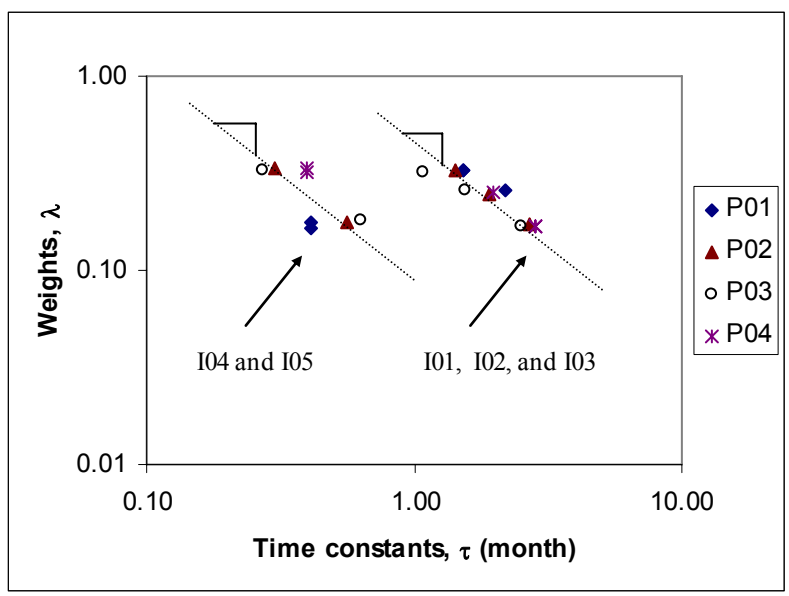

Figure 8 Log-log plot of $\lambda$ 's versus $\tau$ 's. The data separate into two groups where each group gives an approximate straight line of slope -1 . $5 \times 4$ Synfield, high permeability layer.
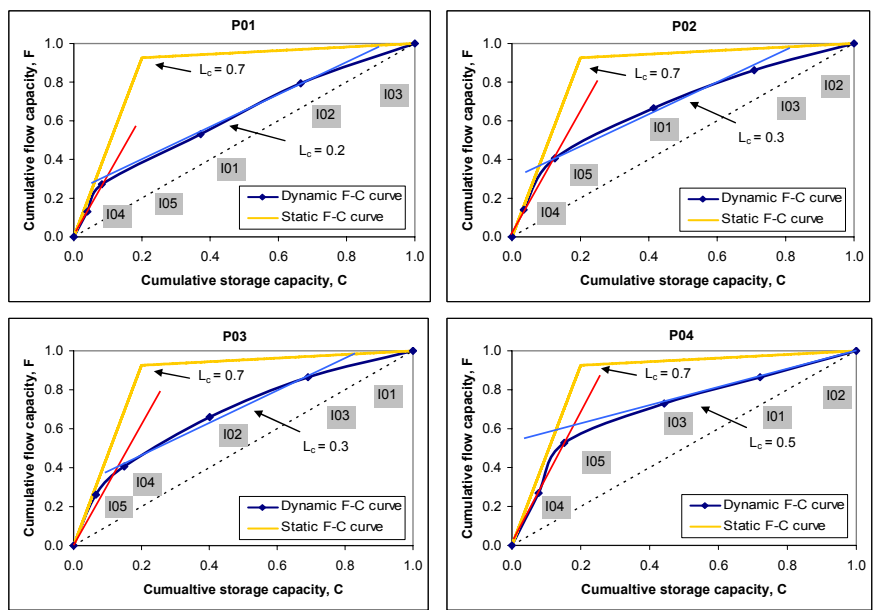

Figure 9: The flow capacity plots for all producers. Each plot shows two different geological layers represented by two straight lines. The dashed line presents the $45^{\circ}$ line. The static flow capacity curve of the field is also depicted in each plot. The $L_{c}$ is the Lorenz coefficient. $5 \times 4$ Synfield, high permeability layer. 


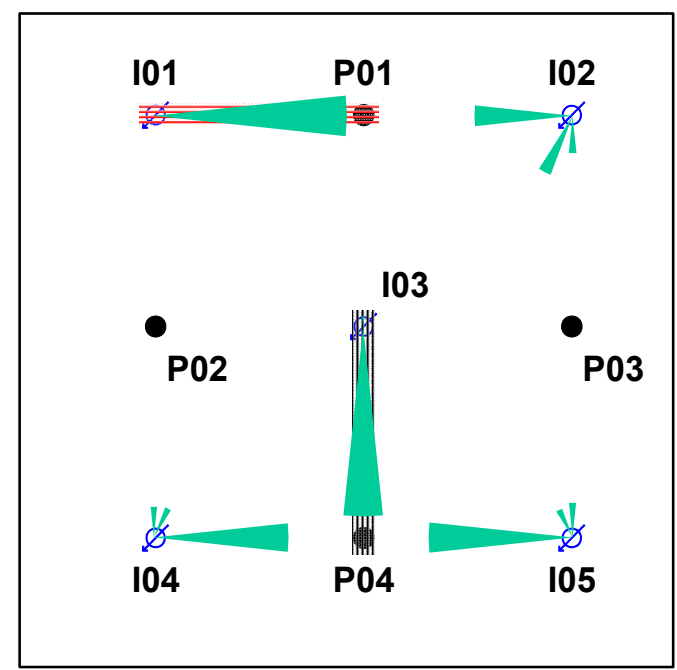

Figure 10: Representation of the weights $\lambda_{\mathrm{ij}}$. The length of the arrow is proportional to the value of $\lambda$. The shaded areas represent the fractures. $5 \times 4$ Synfield, fractures.

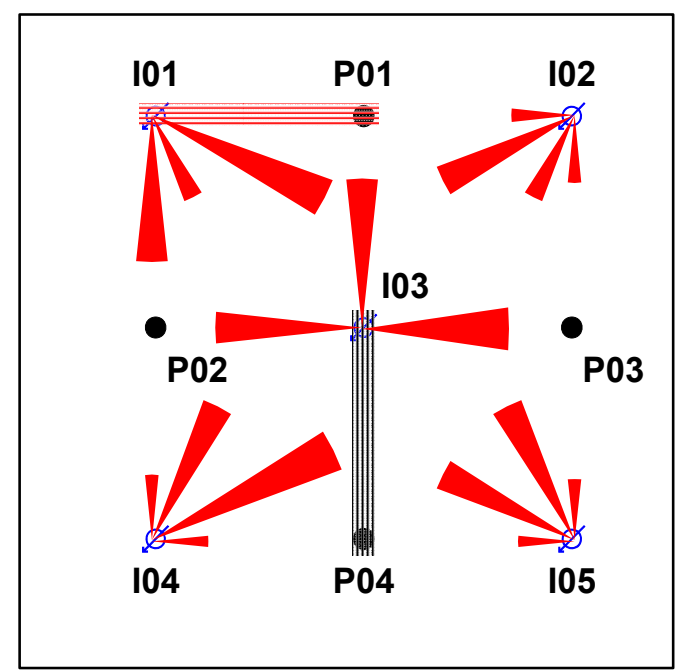

Figure 11: Representation of the time constants $\tau_{\mathrm{ij}}$ (month). The length of the arrow is proportional to the value of $\tau$. The shaded areas represent the fractures. $5 \times 4$ Synfield, fractures. 


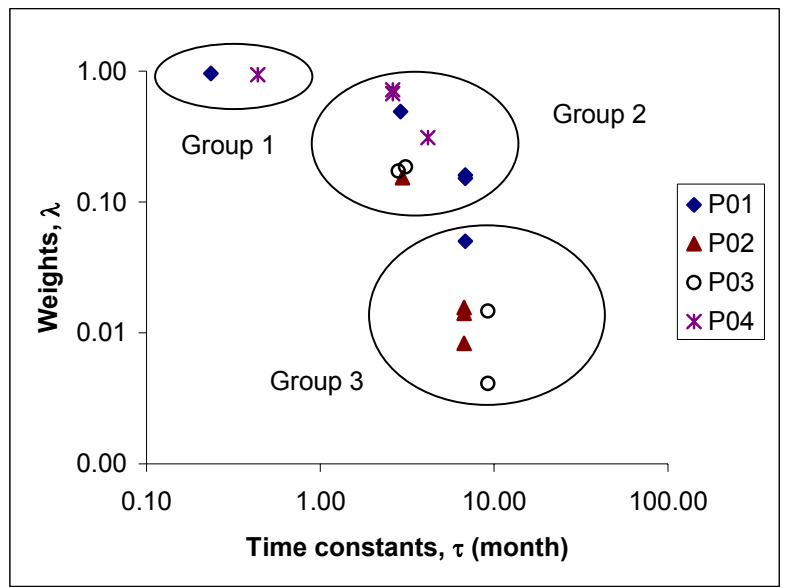

Figure 12: Log-log plot of $\lambda$ 's versus $\tau$ 's. The data indicate three groups. $5 \times 4$ Synfield, fractures.
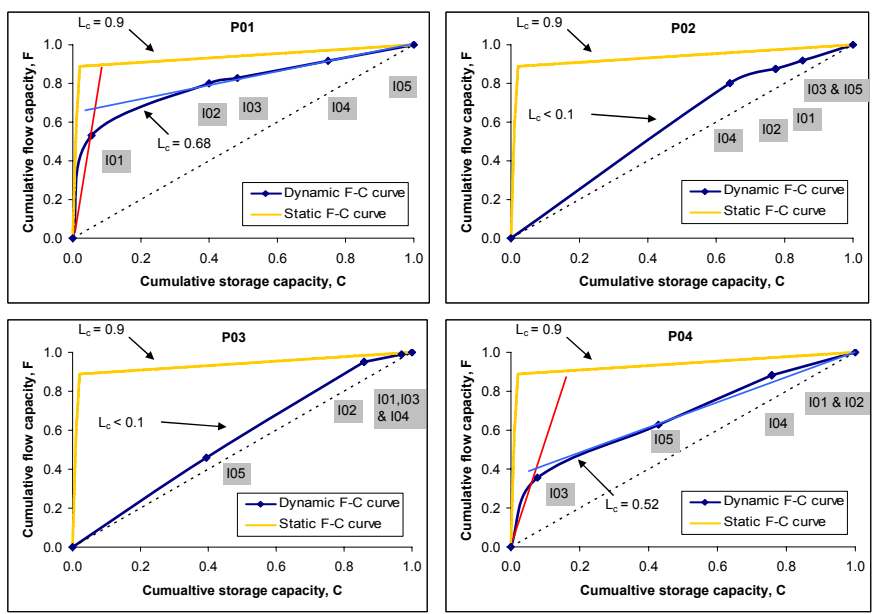

Figure 13: The flow capacity plots for all producers. The plots of P01 and P04 show two distinct geological conditions represented by the two straight lines. The dashed line presents the $45^{\circ}$ line. The static flow capacity curve of the field is also depicted in each plot. The $L_{c}$ is the Lorenz coefficient. 5x4 Synfield, fractures. 


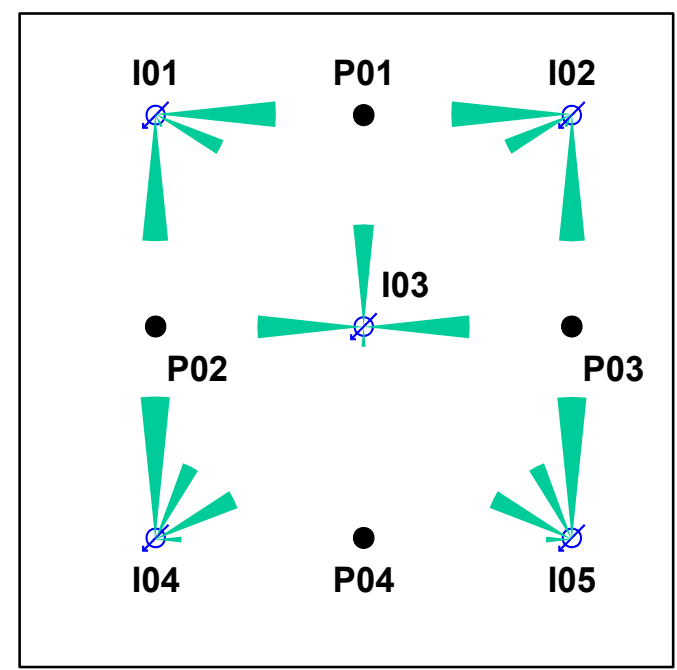

Figure 14: Representation of the weights $\lambda_{\mathrm{ij}}$. The length of the arrow is proportional to the value of $\lambda$. $5 \times 4$ Synfield, producer with partial completion.

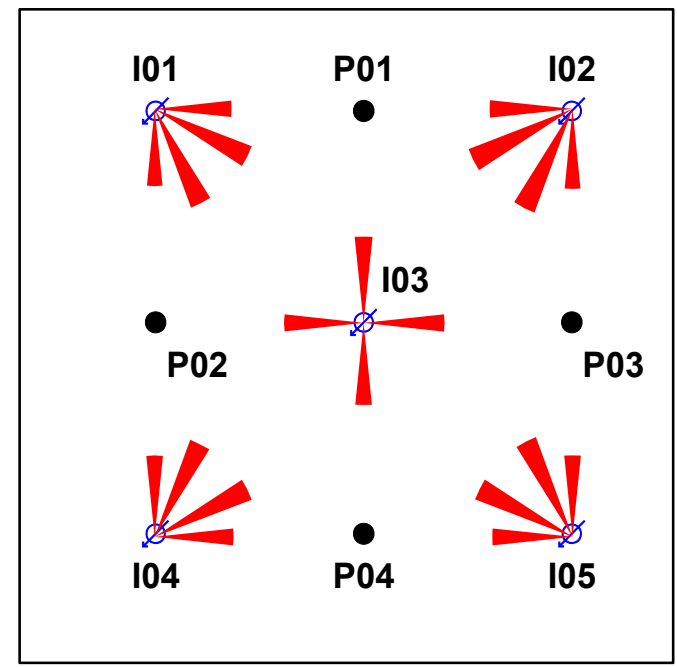

Figure 15: Representation of the time constants $\tau_{\mathrm{ij}}$. The length of the arrow is proportional to the value of $\tau$. $5 \times 4$ Synfield, producer with partial completion. 


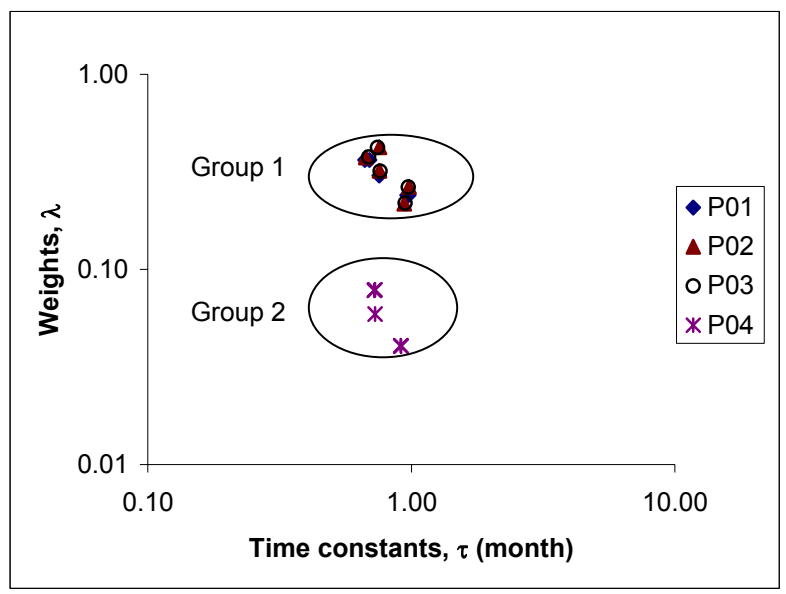

Figure 16: Log-log plot of $\lambda$ 's versus $\tau$ 's. The data indicate two distinct groups. 5x4 Synfield, producer with partial completion.
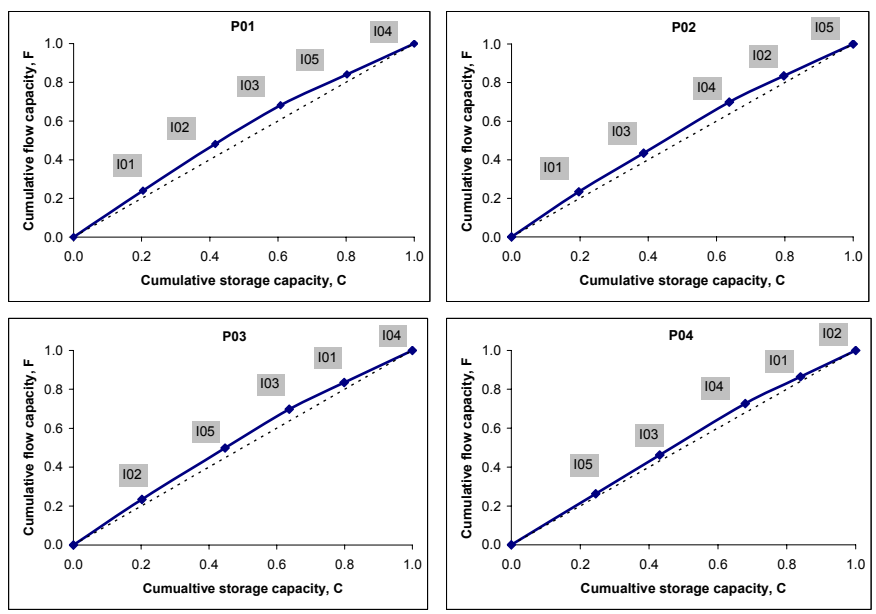

Figure 17: The flow capacity plots for all producers. All plots show a homogeneous reservoir flow capacity. The dashed line presents the $45^{\circ}$ line. $5 \times 4$ Synfield, producer with partial completion. 


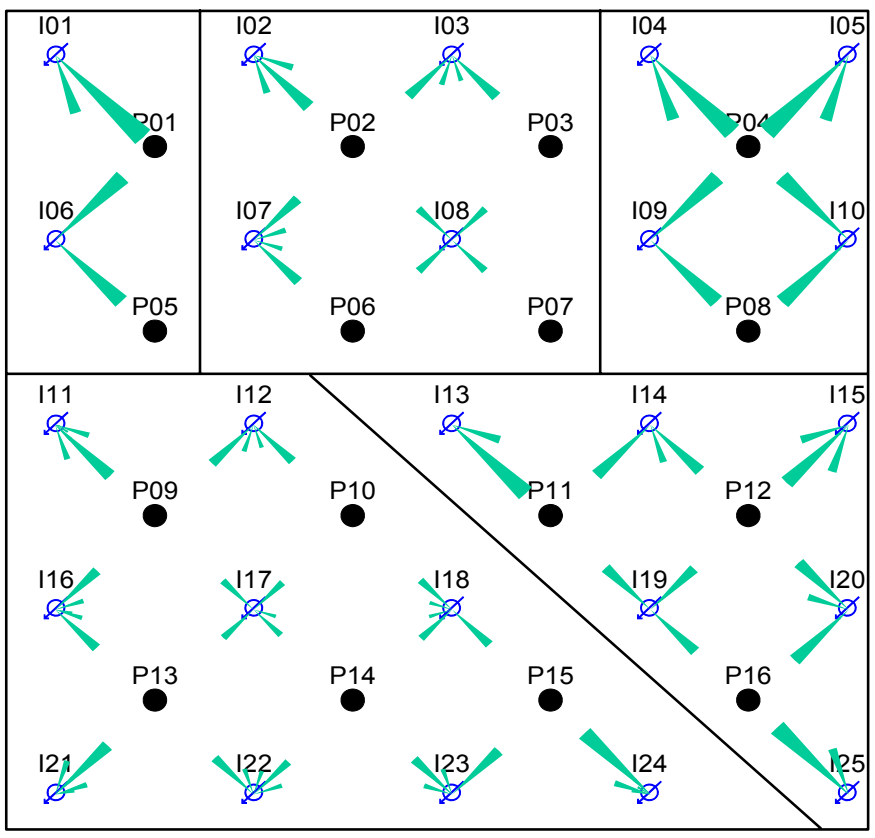

Figure 18: Representation of the weights $\lambda_{\mathrm{ij}}$. The length of the arrow is proportional to the value of $\lambda$. The solid black lines represent the imposed faults. $25 \times 16$ Synfield, reservoir with sealing faults.

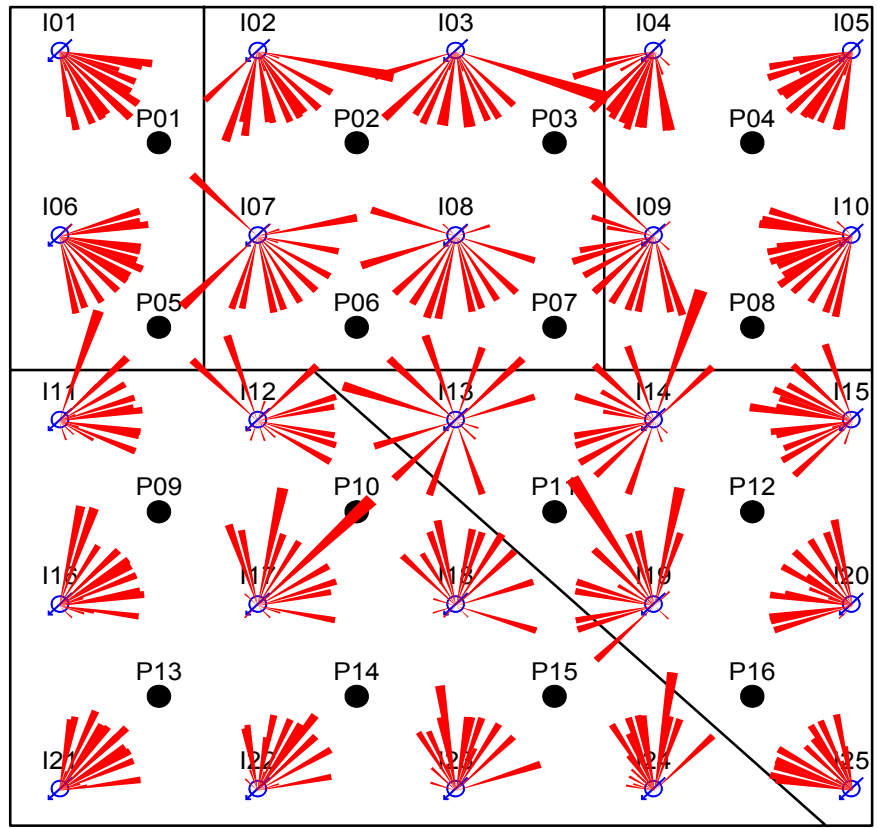

Figure 19: Representation of the time constants $\tau_{\mathrm{ij}}$. The length of the arrow is proportional to the value of $\tau$. The solid black lines represent the imposed faults. $25 \times 16$ Synfield, reservoir with sealing faults. 


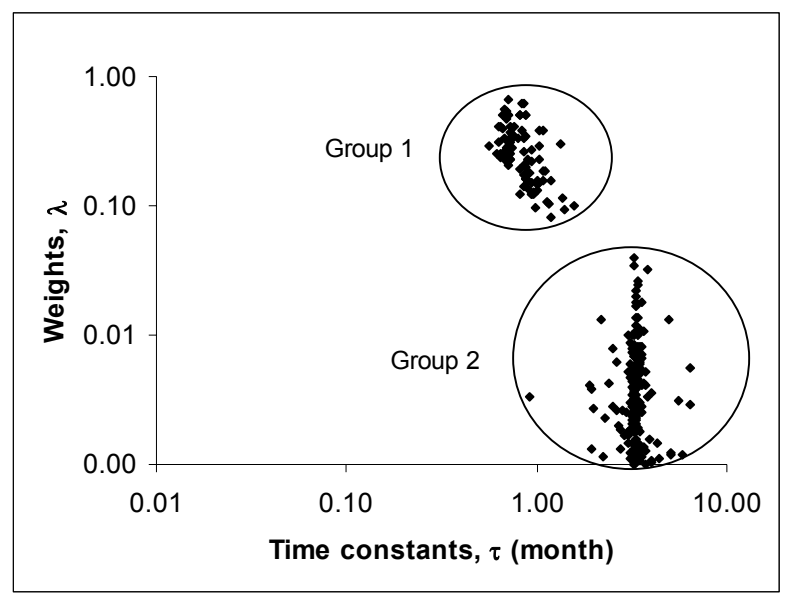

Figure 20: Log-log plot of $\lambda$ 's versus $\tau$ 's for all producers. The data indicate two distinct groups. 25x16 Synfield, reservoir with sealing faults.
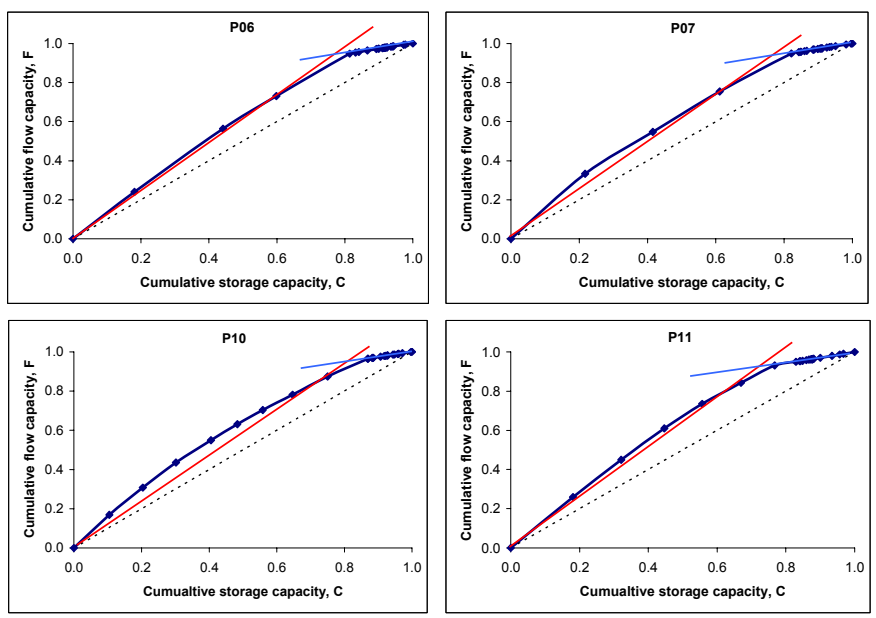

Figure 21: The flow capacity plots for the four center producers. All plots show two distinct geological conditions represented by the two straight lines. The dashed line presents the $45^{\circ}$ line. $25 \times 16$ Synfield, reservoir with sealing faults. 

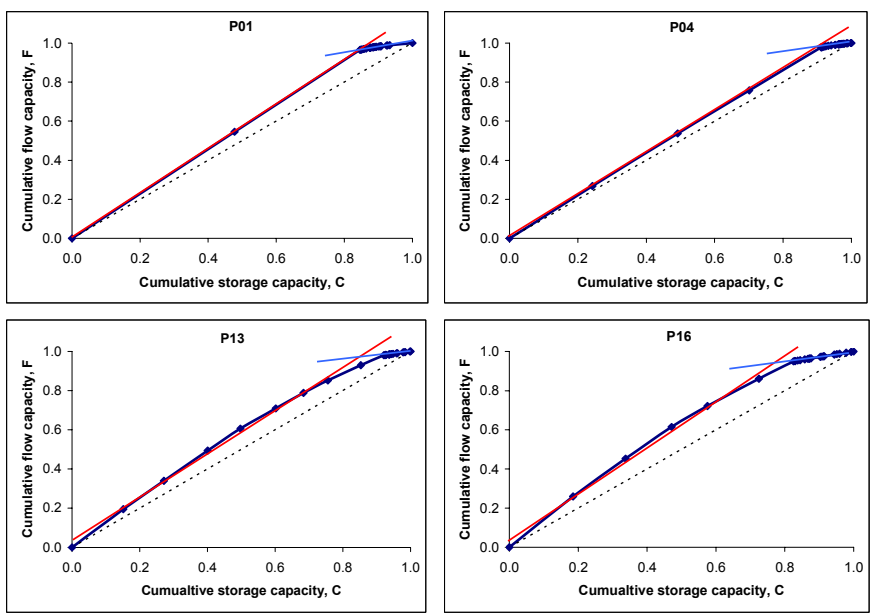

Figure 22: The flow capacity plots for the outer producers. All plots show two distinct geological conditions represented by the two straight lines. The dashed line presents the $45^{\circ}$ line. $25 \times 16$ Synfield, reservoir with sealing faults.

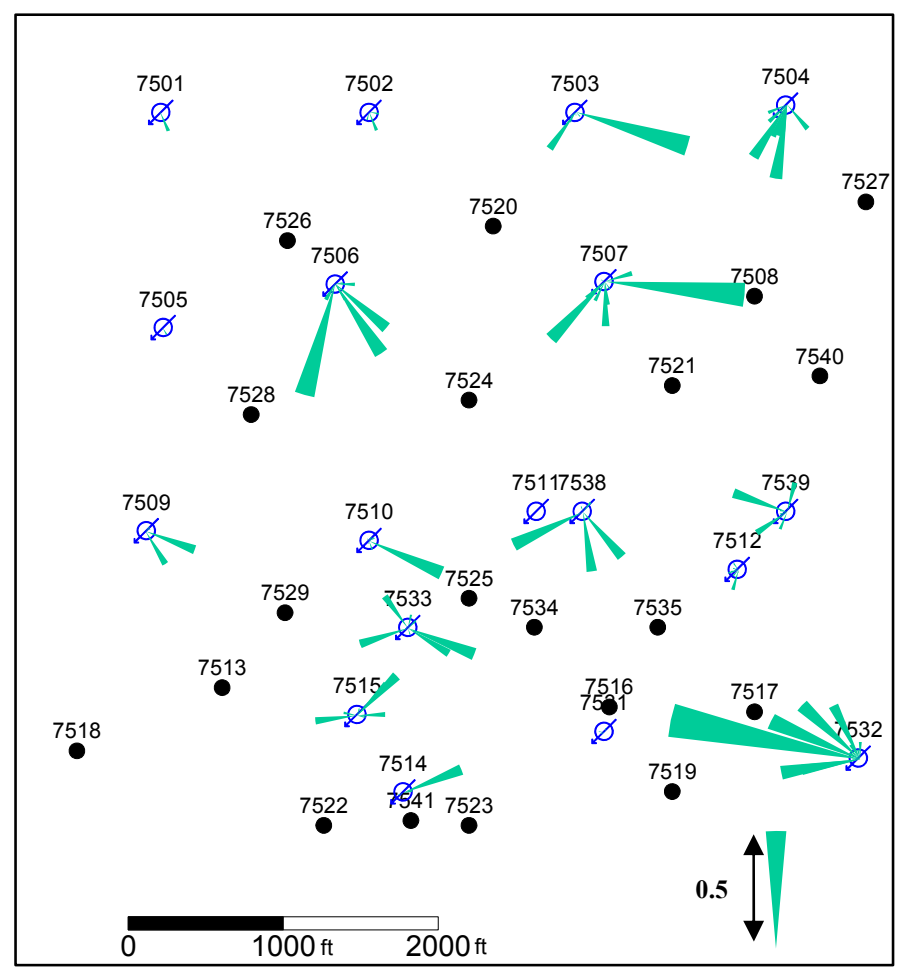

Figure 23: Application of UCM to the SWCF field. Representation of the positive $\lambda$ 's. 


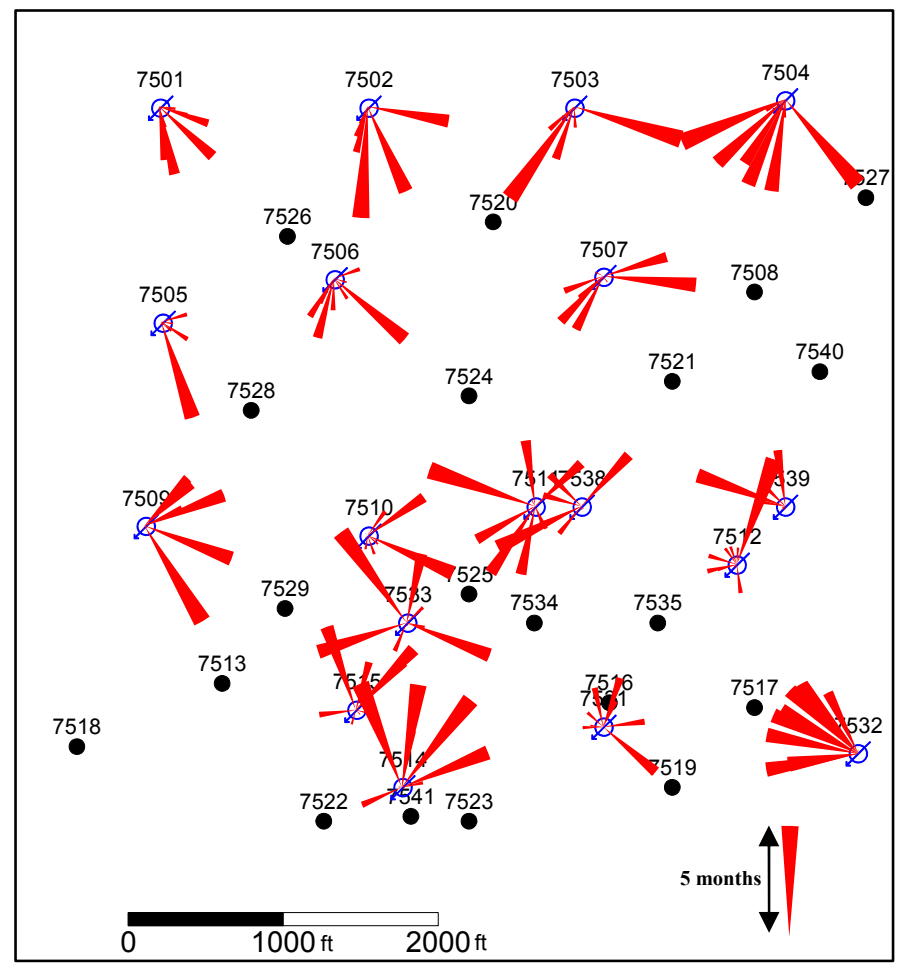

Figure 24: Application of UCM to the SWCF field. Representation of the $\tau$ 's (month).

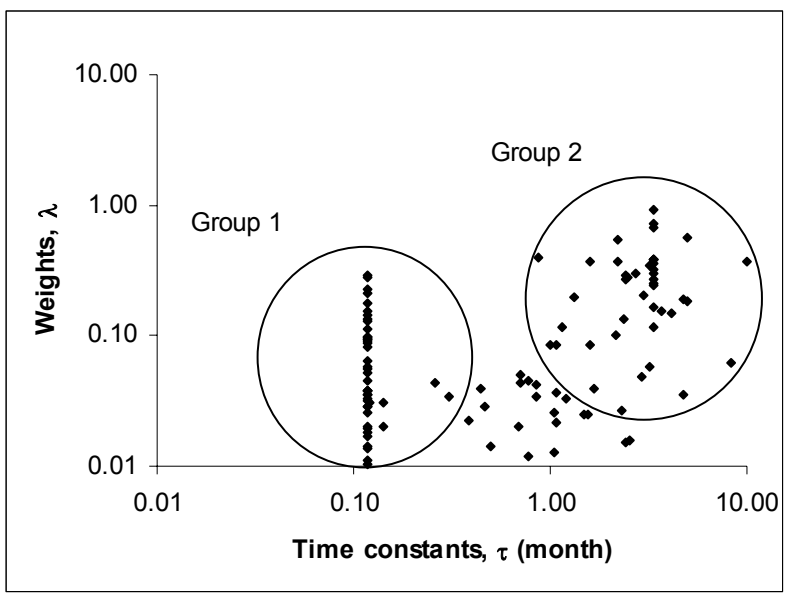

Figure 25: Log-log plot of positive $\lambda$ 's versus $\tau$ 's for all producers in the SWCF field. The data indicate two groups. 

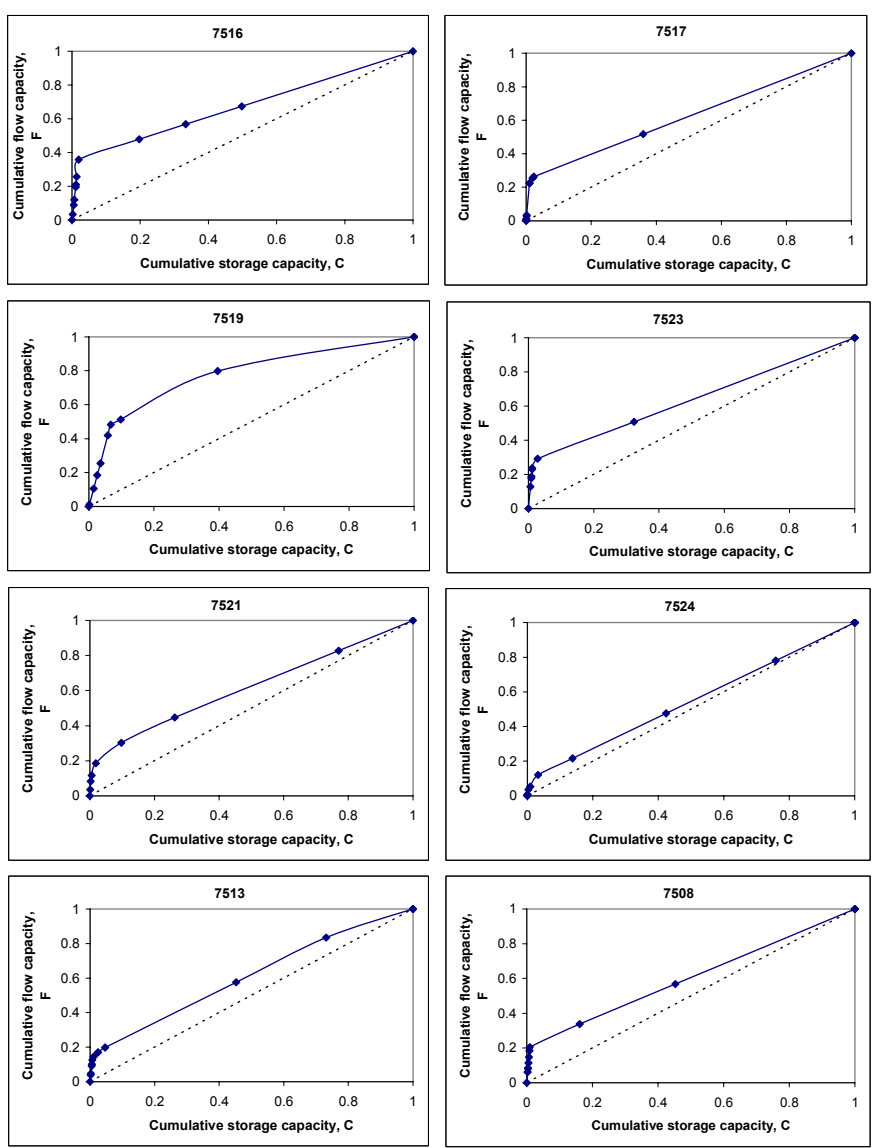

Figure 26: The flow capacity plots for producers well supported by injectors 7506 , 7507, and 7532. The SWCF field. 


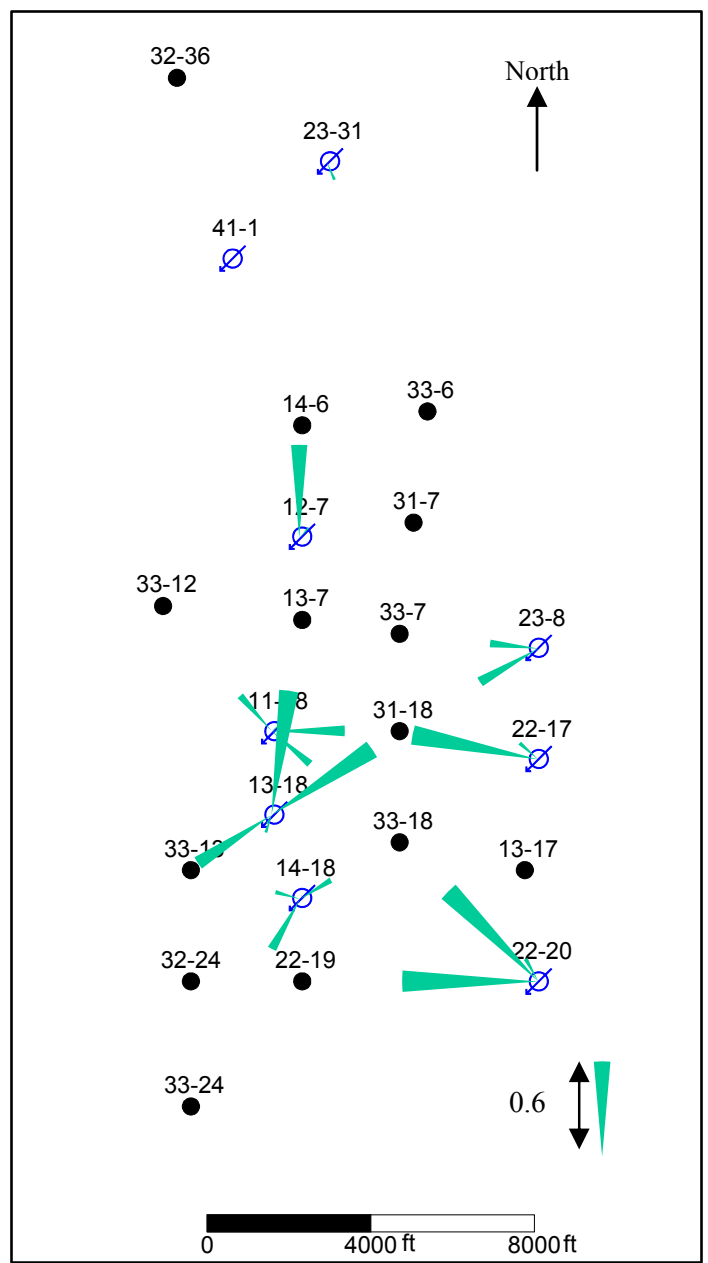

Figure 27: Application of UCM to the NBD field. Representation of the positive $\lambda$ 's. 


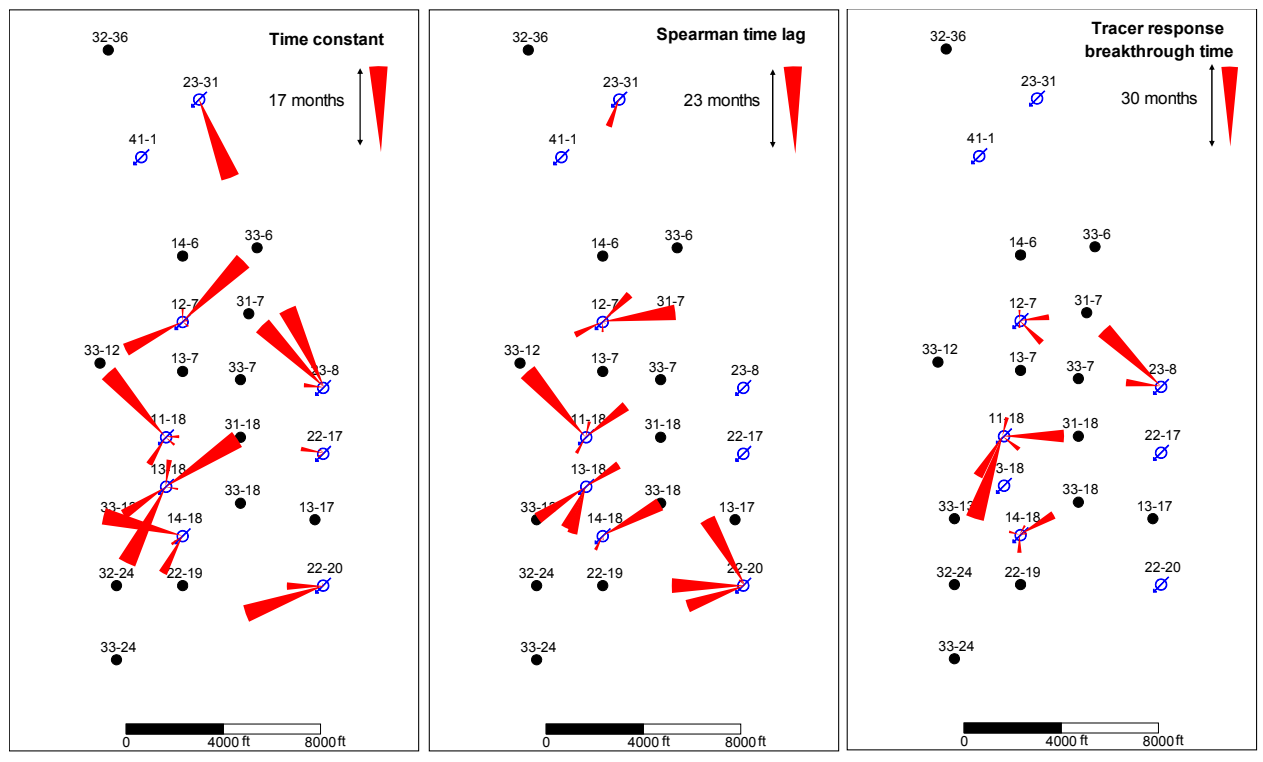

Figure 28: The time constants $(\tau$ 's), the Spearman time lags, and the tracer response breakthrough times for the NBD field. The first plot is for the $\tau$ 's, the second plot is for the Spearman time lags reported by Refunjol and Lake (1997), and the third plot is for the tracer response breakthrough times reported by Refunjol and Lake (1997). 

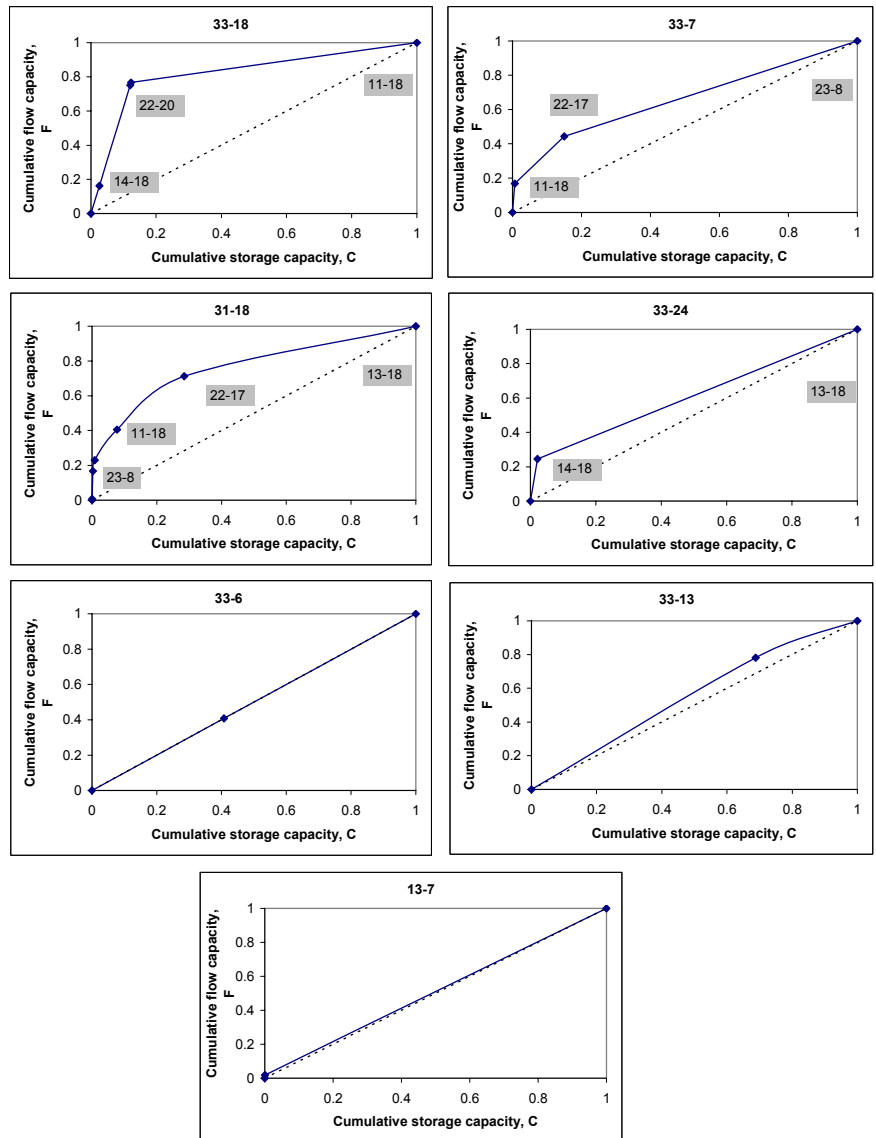

Figure 29: The flow capacity plots using the $\mathrm{CM}$ results for some producers in the NBD field.

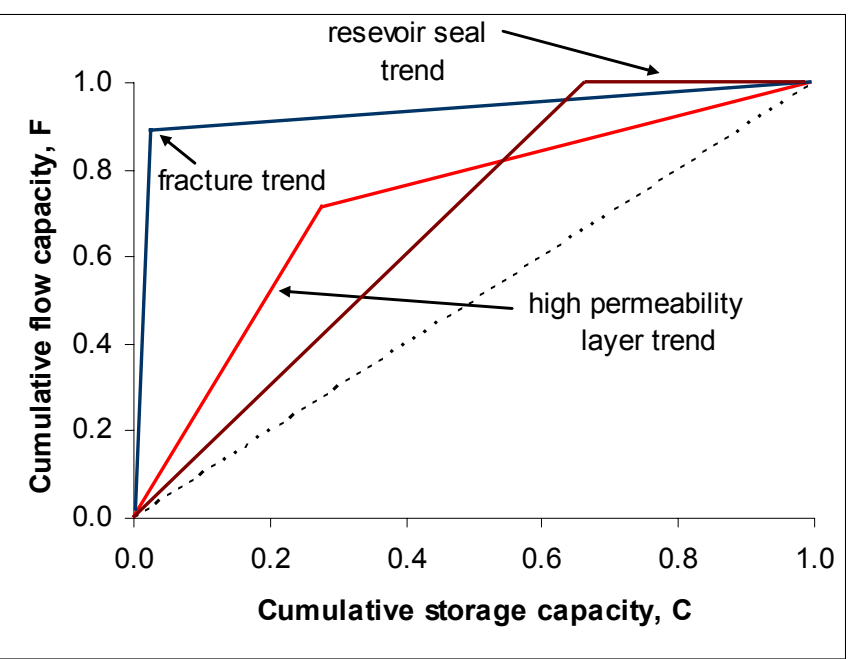

Figure 30: Different trends of the flow capacity curve estimated from the CM parameters according to the corresponding geological feature present in the vicinity of a producer. 


\section{RESULTS AND DISCUSSION \\ PART 2. WATERFLOOD OPTIMIZATION USING CAPACITANCE MODEL RESULTS}

\subsection{Introduction}

Managing production of an oil reservoir to maximize the future economic return of the asset is very important. The techniques to analyze past performance and then to predict the future vary from an educated guess to complex numerical approximations. Most models rely on fitting or matching of historical data.

In petroleum reservoirs, oil production is often constrained by the reservoir conditions, flow characteristics of the pipeline network, fluid-handling capacity of surface facilities, safety and economic considerations, or a combination of these considerations (Kosmidis et al., 2004; Wang et al., 2002a). While adjusting well production rates and allocating waterinjected rates can control production, implementation of these controls in an optimal manner is difficult. Determination of the optimal operating conditions at a given time, subject to all constraints, is the objective of dynamic oil production optimization. This requires simultaneous consideration of the interactions between the reservoir, the wells, and the surface facilities. Typically, one maximizes the oil production rates or minimizes production costs.

Various aspects of oil production optimization have been addressed (Kosmidis et al., 2004; Wang et al., 2002a; Kosmidis et al., 2005) and can be classified into three categories: (1) sensitivity analysis using simulation, (2) heuristic methods, and (3) mathematical programming methods. Using heuristic rules and single well analysis, Barnes et al. (1990) and Stoisits et al. (1992) have studied the allocation of a limited amount of available gas lift wells. Martinez et al. (1994) and Buitrago et al. (1996) applied various mathematical programming techniques such as quasi-Newton and genetic algorithms. Fang and Lo (1996) proposed a linear programming model based on separable programming techniques to allocate gas lift and well rates subject to multiple flow rate constraints. Dutta-Roy and Kattapuram (1997) analyzed a gas lift optimization problem with two wells sharing a common flow line. They pointed out that when flow interactions among wells are significant, nonlinear optimization tools are required. Litvak et al. (1997) developed an integrated reservoir and pipeline network model and employed heuristic rules to allocate well connections to manifolds. Wang et al. (2002b) proposed a linear programming model for a well scheduling problem. To consider the allocation of wells to manifolds, they developed a partial enumeration procedure where only a subset of wells was allowed to switch to different manifolds.

Production and injection rates are the most abundant data available in any injection project. One can analyze these data to obtain information about interwell connectivity. The resulting information may be used to optimize subsequent oil recovery by changing injection patterns, assigning priorities in operations, recompletion of wells, and in-fill drilling.

Several methods have been used to compare the performance of a producing well with that of surrounding injectors. Albertoni and Lake (2003) used a linear model to estimate interwell connectivity in a reservoir using only total production and injection rate data. The coefficients estimated by multiple linear regressions quantitatively indicate the communication between producer and injectors in a waterflood. Filters were adopted to account for time lags between producer and injector.

Gentil (2005) explored the physical meaning of the weights and proposed a new way to interpret them. These insights are used to better understand the underlying assumptions of the model used by Albertoni and Lake and to construct a procedure for incorporating production data into geostatistical permeability distribution models. 
Yousef et al. (2005) used a more complicated model than those in Albertoni and Lake (2003) and Gentil (2005) that includes capacitance (compressibility) as well as resistive (transmissibility) effects. Two coefficients are determined for each injector-producer pair: one parameter (a weight) quantifies the connectivity and the other (a time constant) quantifies the degree of fluid storage between the wells.

None of the previous papers provide an optimization procedure that optimizes the value of oil produced and the cost of the water injected or the water disposal cost. As a result, to optimize performance, oil production rates and water injection rates must be optimized simultaneously with well interconnections. The corresponding optimization problem is considered in this work.

As in Albertoni and Lake (2003), Gentil (2005) and Yousef et al. (2005), the reservoir is considered as a system that converts inputs (monthly injection rates) into outputs (monthly oil and water production rates). Compared with Yousef et al. (2005), a simpler capacitance model is considered first, where weights are calculated to indicate the connectivity between each injector-producer pair and time constants are determined to indicate the degree of fluid storage around each producer. Based on a power law relationship between the instantaneous water-oil ratio and the cumulative water injected, an oil production model with parameter pair $a$ and $b$ for each producer is then proposed. Our models and optimization formulation have been applied to numerically simulated data (Eclipse model) on a heterogeneous synthetic field with five injectors and four producers.

In section 2, we present the capacitance model to indicate the connectivity and dissipation between wells. Section 3 contains an oil production model showing the relationship between the instantaneous water-oil ratio and the cumulative water injected. In section 4 , various optimization problems for oil production are formulated to maximize the future economic return of the reservoir asset. All models and formulation are tested on a heterogeneous synthetic field with five injectors and four producers and the results are shown in section 5. Conclusions are drawn in section 6.

\subsection{Capacitance Model}

The capacitance model is a total mass balance with compressibility (Yousef et al., 2005). In a real waterflood, there are multiple producers and injectors acting simultaneously and more than one injector usually influences the total production rate at a producer (Yousef et al., 2005). In this work, we consider a drainage volume with $m$ injectors and $n$ producers.

The governing material balance at reservoir conditions can be described by the following differential equations:

$$
q_{j}=\sum_{k=1}^{m} \lambda_{k j} i_{k}-c_{t} V_{p} \frac{d \bar{p}_{j}}{d t} \quad j=1,2, \cdots, n
$$

where $c_{t}$ is the total compressibility; $V_{p}$ is the original pore volume of the drainage; $\bar{p}_{j}$ is the average pressure drained by producer $j$; $i_{k}$ is the injection rate in injector $k$ and $q_{j}$ is the total production rate in producer $j$. To obtain a description that is based entirely on rates, as Yousef et al.(2005) did, we use the same linear productivity model

$q_{j}=J_{j}\left(\bar{p}_{j}-p_{w f j}\right) \quad j=1,2, \cdots, n$

where $p_{w f j}$ and $J_{j}$ are the flowing bottomhole pressure and productivity index of the producer $j$, respectively. Eliminating $\bar{p}_{j}$ in Equations (1) and (2), we obtain

$$
q_{j}=\sum_{k=1}^{m} \lambda_{k j} i_{k}-\frac{c_{t} V_{p}}{J_{j}} \cdot \frac{d q_{j}}{d t}-c_{t} V_{p} \frac{d p_{w f j}}{d t} \quad j=1,2, \cdots, n
$$

In this work, we assume a constant flowing bottomhole pressure for simplicity. By defining $\tau_{j}=c_{t} V_{p} / J_{j}$, we obtain the following capacitance model 
$q_{j}=\sum_{k=1}^{m} \lambda_{k j} i_{k}-\tau_{j} \frac{d q_{j}}{d t} \quad j=1,2, \cdots, n$

This model provides one time constant $\tau_{j}$ for each producer $j$ and one weight $\lambda_{k j}$ for each injector-producer $(k j)$ pair, which is simpler than that in Yousef et al. (2005). The $m \times n$ weights provide a quantitative expression of the connectivity between each injector- producer pair; the larger the weight, the greater the connectivity. The $n$ time constants are direct measures of the rate of dissipation around each producer; the larger the time constant, the larger the dissipation rate.

The oil production model is based on a power law relationship between the instantaneous water-oil ratio (WOR) and the cumulative water injected (Wi). The predicted water-oil ratio is assumed to have the following power law form:

$$
\mathrm{WOR}=a \mathrm{Wi}^{b}
$$

Once injection and production rates are in balance, total fluids produced approximate Wi and $\mathrm{Wi}$ is the approximate sum of the cumulative oil and water produced. Under this circumstance, equation (5) can be applied for each producer. Hence, any multivariate linear regression method can be used to obtain a total liquid prediction rate without explicit information regarding the origin of the water injected.

Assuming that the capacitance models provide information on how the water is being allocated from the injectors to the producers, a WOR-WI power law can be applied to each individual volume drained by each producer after solving for the capacitance model weights. The total oil rate produced by producer $j$ is the total production rate multiplied by the oil fraction $f_{i}$ at that time level:

$$
q_{o j}=f_{j} q_{j} \quad j=1,2, \cdots, n
$$

The producing oil fraction is calculated from the estimated water-oil ratio as:

$$
f_{j}=\frac{1}{1+\mathrm{WOR}_{j}} \quad j=1,2, \cdots, n
$$

A power law is used to model the water-oil ratio as a function of the total cumulative water injected for each producer $j$ :

$$
\mathrm{WOR}_{j}=a_{j} \mathrm{Wi}_{j}^{b_{j}} \quad j=1,2, \cdots, n
$$

The total cumulative water injected into the drainage area of producer $j$ is:

$$
\mathrm{Wi}_{j}=\int_{s=t_{0}}^{s=t}\left[\sum_{k=1}^{m} \lambda_{k j} i_{k}(s)\right] d s=\sum_{k=1}^{m} \lambda_{k j} \int_{s=t_{0}}^{s=t} i_{k}(s) d s
$$

Thus, we can express the oil production from region $j$ as follows.

$$
q_{o j}=\frac{q_{j}}{1+a_{j}\left(\sum_{k=1}^{m} \lambda_{k j} \int_{s=t_{0}}^{s=t} i_{k}(s) d s\right)^{b_{j}}}, j=1,2, \cdots, n
$$

After estimating the weights in the capacitance model, we can use Eq.(10) model to determine the parameter pair $a_{j}$ and $b_{j}$ for each producer by minimizing the squared errors between measured historical oil production rates and those generated by the discrete form of Equation (10). However, direct use of the above power-law model form makes it difficult to obtain accurate value of $a_{j}$ and $b_{j}$. So, taking logarithms to linearize Equation (8):

$$
\log \left(\mathrm{WOR}_{j}\right)=\log \left(a_{j}\right)+b_{j} \log \left(\mathrm{Wi}_{j}\right)
$$


Using linear regression, $\log \left(a_{j}\right)$ and $b_{j}$ are found by minimizing the relative sum of squared errors (note the errors are now in terms of logarithms). Naturally, linearization of Equation (8) comes at a price and some care must be taken when using it. It is recommended that only late time data be used for fitting, particularly if it shows a clear trend on a WOR-Wi cross plot.

Formulation of the problem is very important in the practical use of optimization techniques. Possible objectives can be to maximize the total oil flow from the field or minimize the profitability of the reservoir. For maximizing the total oil production over the period $\left[t_{0}, t_{f}\right]$, we can use the following objective function.

$$
Q_{o}=\sum_{j=1}^{n} \int_{s=t_{0}}^{s=t_{f}} q_{o j}(s) d s
$$

To maximize the non-discounted revenue over the period $\left[t_{0}, t_{f}\right]$, we use the objective function

$$
R=p_{o} \sum_{j=1}^{n} \int_{s=t_{0}}^{s=t_{f}} q_{o j}(s) d s-p_{w} \sum_{k=1}^{m} \int_{s=t_{0}}^{s=t_{f}} i_{k}(s) d s
$$

where the coefficients $p_{o}$ and $p_{w}$ are the prices of oil and water injected, respectively. We can also consider the following objective function as the non- discounted revenue over the period $\left[t_{0}, t_{f}\right]$

$$
R=p_{o} \sum_{j=1}^{n} \int_{s=t_{0}}^{s=t_{f}} q_{o j}(s) d s-\bar{p}_{w} \sum_{j=1}^{n} \int_{s=t_{0}}^{s=t_{f}} q_{w j}(s) d s
$$

where $p_{o}$ is the price of oil and $\bar{p}_{w}$ is the cost of water disposed. Another objective function can be based on net present value; weighting is applied to cash flows in each time period to account for net present values.

The decision variables include the well production rates $q_{j}(t), j=1,2, \cdots, n$, and the well injection rates $i_{k}(t), k=1,2, \cdots, m$. The constraints include the capacitance model (4), oil production model (10) and some upper $\left(u_{k}\right)$ and lower $\left(l_{k}\right)$ bounds imposed on the injection rates for the $m$ injectors.

$$
l_{k} \leq i_{k}(t) \leq u_{k}, \quad k=1,2, \cdots, m
$$

The minimum $l_{k}$ usually is zero, which occurs when injector $k$ is shut in. The maximum value is user specified. Now we can choose the optimal injection rates and production rates by solving one of the following optimization problems over the period $\left[t_{0}, t_{f}\right]$ :

Maximize $Q_{o}$ in (12) or $R$ in (13) or (14)

Subject to $q_{j}=\sum_{k=1}^{m} \lambda_{k j} i_{k}-\tau_{j} \frac{d q_{j}}{d t}$

$$
q_{o j}=\frac{q_{j}}{1+a_{j}\left(\sum_{k=1}^{m} \lambda_{k i} \int_{s=t_{0}}^{s=t} i_{k}(s) d s\right)^{b_{j}}}, \quad j=1,2, \cdots, n
$$

$l_{k} \leq i_{k}(t) \leq u_{k}, \quad k=1,2, \cdots, m$

This optimization problem formulation will be converted into a standard nonlinear constrained programming problem via discretization (Edgar et al., 2001), which can be solved by an efficient algorithm such as sequential quadratic programming (SQP). However, the size 
of the discretized problem depends on the number of wells, the length of the period $\left[t_{0}, t_{f}\right]$ and the step size of discretization. It may have a large number of variables. Therefore, large-scale optimization techniques may be necessary to solve the problem.

\subsection{Results}

To show the utility of the capacitance model, oil production model and optimization formulation for oil production, we applied them to numerically simulated data (Eclipse) on a heterogeneous synthetic field (a Synfield, see Yousef, 2006 for the details). The results of these applications are presented and discussed below.

The Synfield consists of 5 injectors and 4 producers as shown in Figure 1. The field has only vertical wells. The Synfield is a multi-layered anisotropic reservoir with a homogenous permeability of $5 \mathrm{md}$ except for two streaks: one between I01 and P01 $(1000 \mathrm{md})$, the other between I03 and P04 (500 md). The Synfield dimensions and the grid size are $31 \times 31 \times 5$ and $40 \times 40 \times 6 \mathrm{ft}$, respectively. The distance between nearest injector and producer is $800 \mathrm{ft}$. The oil-water mobility ratio is equal to one and the oil, water and rock compressibilities are $5 \times 10^{-}$ ${ }^{6}, 1 \times 10^{-6}$ and $1 \times 10^{-6} \mathrm{psi}^{-1}$, respectively. The flowing bottomhole pressures of the producer are constant. We used the same injection data as Yousef (2006). These data, as shown in Figures 2a-e, were randomly selected from different wells in a real field and proportionally modified to be in agreement with the Synfield injectivity. The numerical simulation extends for 100 months (approximately 3000 days), which represent a history of 100 data points of production. The corresponding total production rate, oil production rate and water production rate for the four producers are shown in Figures 3a-d.

The numerical values of the weights and time constants are obtained from the capacitance model by a standard constrained optimization solver in Matlab 7.1. These are shown in Tables 1 and 2. In Figure 4, the weights $\lambda_{k j}$ are represented by arrows or cones that start from injector $k$ and point to producer $j$. The larger the arrow, the larger the value of the weight and the greater the connectivity between the two wells.

Figure 4 reveals different characteristics of the medium between each injector and producer pair. As expected from a heterogeneous reservoir, the weights are not symmetric. The capacitance model describes the injection-production behavior and the weights quantify the connectivity between wells appropriately. The presence of two streaks, between I01 and P01 and between I03 and P04 as shown in Figure 1, is obvious from the weights. The water injected in injector I01 (I03) almost exclusively flows along the streak to producer P01 (P04). Producer P04 seems to be located within a high permeability area and for injectors I02, I03, I04 and I05, the weighting coefficient affecting P04 is larger than any of the coefficients affecting the other producers.

Figures 5 shows comparisons between the modeled total production rate using the capacitance model and the total production rate observed in the numerical simulation for producer P01. The coefficient of determination is $R^{2}=0.9989$. The agreement of the model prediction for other producers P02, P03 and P04 are not shown; their corresponding coefficients of determination are $R^{2}=0.9992, R^{2}=0.9999$ and $R^{2}=0.9986$. All matches of total production rate in the Synfield yielded $R^{2}>0.99$. As a consequence, we conclude the capacitance model successfully captures the attenuation and time lag in the field studied.

After obtaining the weights and time constants, we then used the oil production model to estimate the parameters $a_{j}$ and $b_{j}$ for each producer. To obtain accurate parameters, we use Equation 11. We eliminated the data in previous 60 months, that is, we only used the latest 40 months of data. A standard unconstrained optimization solver in Matlab 7.1 is used to estimate the parameters $a_{j}$ and $b_{j}$ for each producer; the results obtained are shown in Table 3 . Figures 6a-d show comparisons between the oil production rate modeled and the oil 
production rate observed in the numerical simulation for each producer. The matches of oil production rates in the whole region are not as good as desired, except for producer P02. But for the most resent 40 months, the $R^{2}$ for producers P01-P04 are, respectively, 0.9776, $0.9998,0.9952$ and 0.9928 . Therefore, the matches are quite good and the model yields accurate predictions on oil production.

Before the oil optimization, the lower $\left(l_{k}\right)$ and upper $\left(u_{k}\right)$ bounds imposed on the injection rates for the 5 injectors need to be specified first. For each injector, we take its lower bound as zero and its upper bound as the maximum value of its historical data. That is, we specify the lower and upper values for each injector as the following values (in RB/day):

$l_{k}=0, k=1,2, \cdots, 5$

$u_{1}=3531.916, u_{2}=1963.172, u_{3}=1456.326, u_{4}=1346.799, u_{5}=2209.986$

If we take (12) as the objective function, that is, if we only maximize the total oil production, then the optimal injection rates in all five injectors take their upper extreme values. If we take (13) as the objective of revenue optimization with different prices of oil and water injected, then we can consider different scenarios of injection rates for each injector. In some cases, the oil prices are admittedly exorbitant to see where an injection policy might be changed. Using a fixed water price of $\$ 2 / \mathrm{RB}$ (related to water disposed costs) and increasing the oil price in increments of $\$ 1$ from $\$ 2 / \mathrm{RB}$ to $\$ 600 / \mathrm{RB}$ resulted in the following series of revenue optimization results.

$\diamond$ When the oil price is between $\$ 2-14 / \mathrm{RB}$, all injectors are shut in;

$\diamond$ When the oil price is between $\$ 15-34 / \mathrm{RB}$, all injectors except injector I01 are shut in; injector I01 changes its rate between its lower and upper bounds. See Figure 7a-b as an example where oil price is $\$ 20 / \mathrm{RB}$.

$\diamond$ When the oil price is between $\$ 35-82 / \mathrm{RB}$, injectors I03, I04 and I05 are shut in; injector I01 takes its upper extreme value for all time, and, injector I02 changes its rate between its lower and upper bounds. A case with oil price $\$ 60 / \mathrm{RB}$ is shown in Figure 8a-b.

$\diamond$ When the oil price is between $\$ 83-292 / \mathrm{RB}$, injector I03 is shut in, injector I01 and injector I02 take their upper extreme values, injectors I04 and I05 change their rates between their lower and upper bounds, respectively. Figure 9a-b shows the optimal injection rates for each injector under oil price \$180/RB.

$\diamond$ When the oil price is between $\$ 293-599 / \mathrm{RB}$, all injectors except injector I03 take their upper bounds; injector I03 changes its rate between its lower and upper extreme value. An example with oil price $\$ 400 / \mathrm{RB}$ is shown in Figure $10 \mathrm{a}-\mathrm{b}$.

$\diamond$ When the oil price is equal to or larger than $\$ 600 / \mathrm{RB}$, all injectors take their upper extreme values.

If we take (14) as the objective of revenue optimization with different prices of oil and costs of water disposed, then we can obtain the following optimal injection policy with a fixed $\$ 1 / \mathrm{RB}$ price of water disposed and increasing the oil price in increments of $\$ 1$ from $\$ 1 / \mathrm{RB}$ to $\$ 450 / \mathrm{RB}$.

$\diamond$ When the oil price is between $\$ 1-12 / \mathrm{RB}$, all injectors are shut in;

$\diamond$ When oil price is equal to or larger than $\$ 430 / \mathrm{RB}$, all injectors take their upper extreme values;

$\diamond$ For oil price between $\$ 13-429 / \mathrm{RB}$, it is hard to classify the optimal injection patterns. Four examples with oil price \$20/RB, \$60/RB, \$120/RB and \$240/RB are shown in Figures 11-14.

\subsection{Conclusions}

We have shown an optimization procedure that systematically accounts for the interactions of an integrated oil and water production system and simultaneously optimizes 
the oil produced using injected water. A simpler capacitance model is sufficient to predict the dynamic behavior for injection and production wells in a reservoir, which represent a significant reduction in computational effort compared to that in Yousef et at. (2005). Using historical data, the estimated oil production matches that from a numerical simulation at long time.

Using the simplified reservoir model, we formulated various objective functions for future oil production, where production rates and water injection rates are optimized simultaneously. Solving the proposed optimization problem for different oil prices led to different scenarios of optimal injection.

The models and formulation were applied to numerically simulated data (Eclipse) on a heterogeneous synthetic field with 5 injectors and 4 producers. The results show that the proposed capacitance model can successfully capture the true attenuation and time lag behavior between injectors and producers. The formulation of optimal oil production can be used to maximize the future economic return of the asset and is appropriate for simultaneous optimization of well rates in the field where wells are shut-in frequently. The proposed optimization method can be used for real-time production control because all of the variables required for the model can be measured and the discrete data can be directly incorporated in the formulation.

\subsection{Tables and Figures}

Table 1. Interwell weighs obtained

\begin{tabular}{|c|c|c|c|c|}
\hline & P01 & P02 & P03 & P04 \\
\hline I01 & 0.9374 & 0.0145 & 0.0084 & 0.0397 \\
\hline I02 & 0.2920 & 0.0135 & 0.2632 & 0.4313 \\
\hline I03 & 0.0316 & 0.0057 & 0.0044 & 0.9584 \\
\hline I04 & 0.1024 & 0.1607 & 0.0074 & 0.7295 \\
\hline I05 & 0.0395 & 0.0107 & 0.2168 & 0.7330 \\
\hline
\end{tabular}

Table 2. Time constants (in months) obtained

\begin{tabular}{|c|c|c|c|c|}
\hline & P01 & P02 & P03 & P04 \\
\hline $\boldsymbol{\tau}$ & 0.2372 & 1.4813 & 2.4180 & 1.0438 \\
\hline
\end{tabular}

Table 3. Parameters $a[1 / \mathrm{rb}]$ and $b$ obtained

\begin{tabular}{|c|c|c|c|c|}
\hline & $\mathbf{P 0 1}$ & $\mathbf{P 0 2}$ & $\mathbf{P 0 3}$ & $\mathbf{P 0 4}$ \\
\hline $\boldsymbol{a}$ & $4.416 \mathrm{e}-07$ & $3.551 \mathrm{e}-16$ & $6.462 \mathrm{e}-13$ & $6.486 \mathrm{e}-20$ \\
\hline $\boldsymbol{b}$ & 1.4098 & 3.9002 & 2.7559 & 3.8003 \\
\hline
\end{tabular}




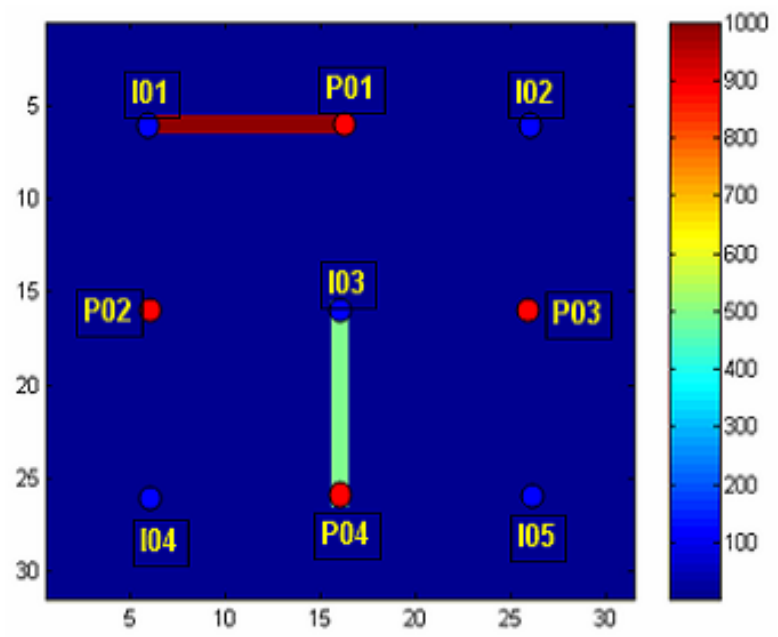

Figure 1. The locations of the vertical wells and permeability (in $\mathrm{md}$ ) in the reservoir from Yousef.

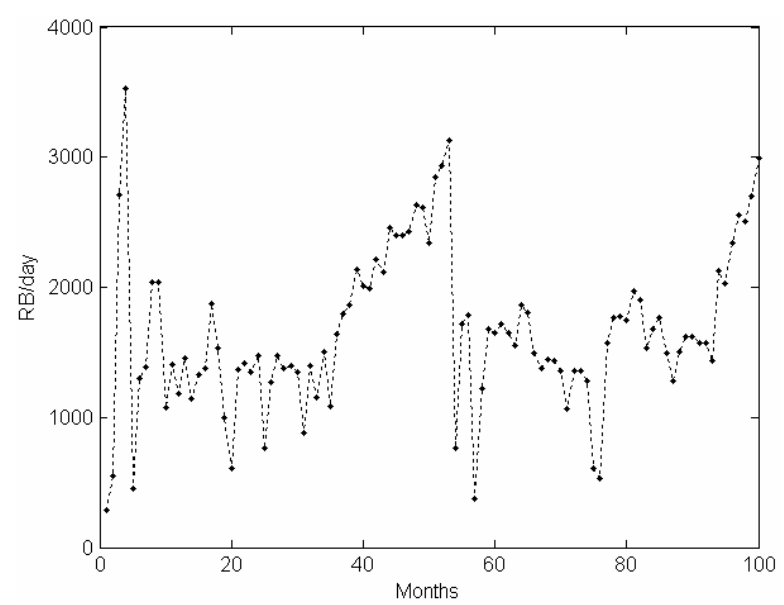

Figure 2a. Rate for injector 101

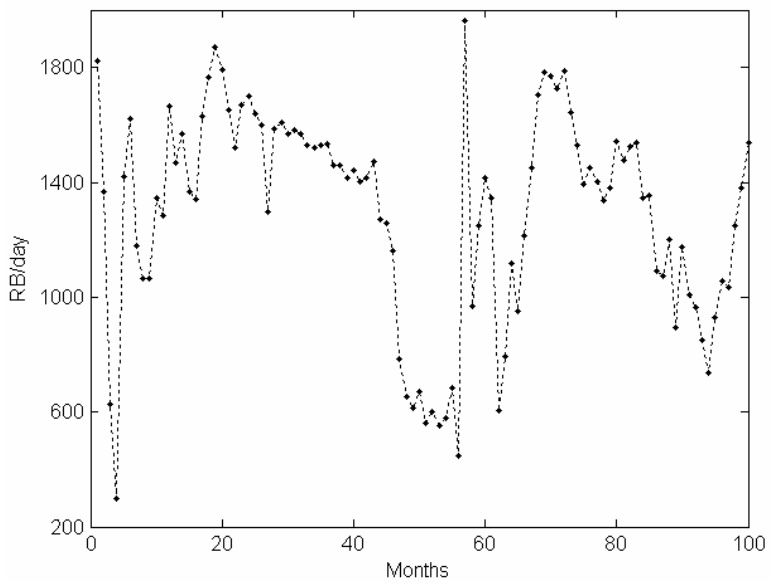

Figure 2b. Rate for injector 102 


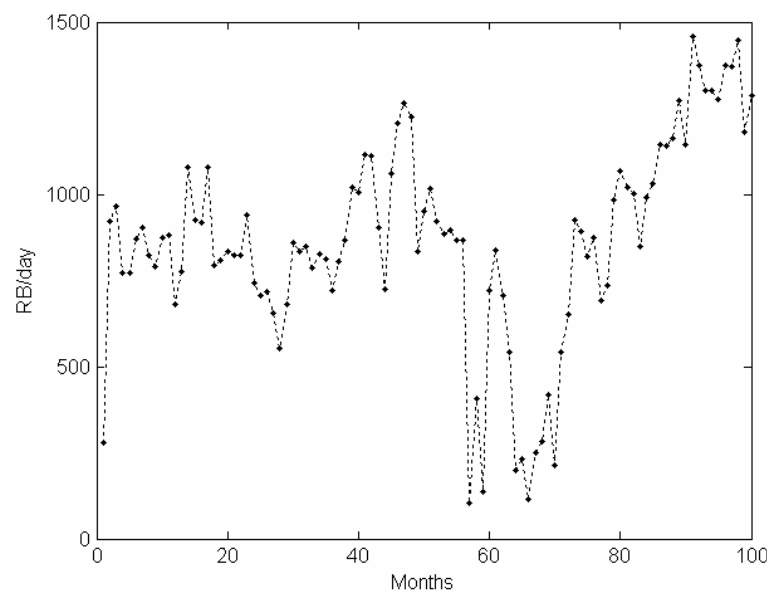

Figure 2c. Rate for injector 103

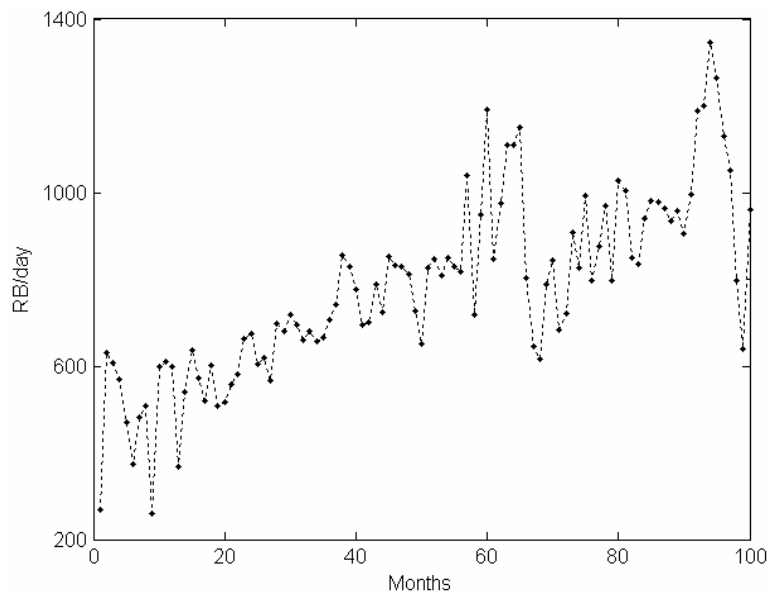

Figure 2d. Rate for injector 104

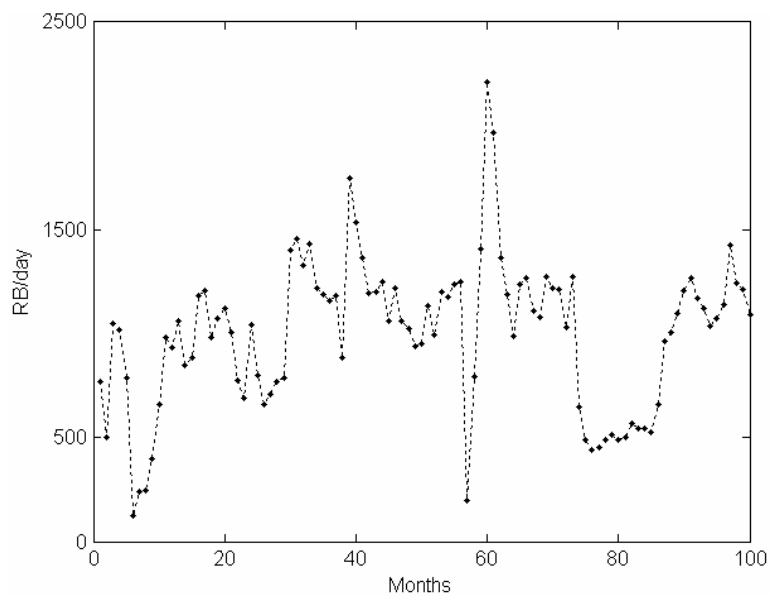

Figure 2e. Rate for injector 105 


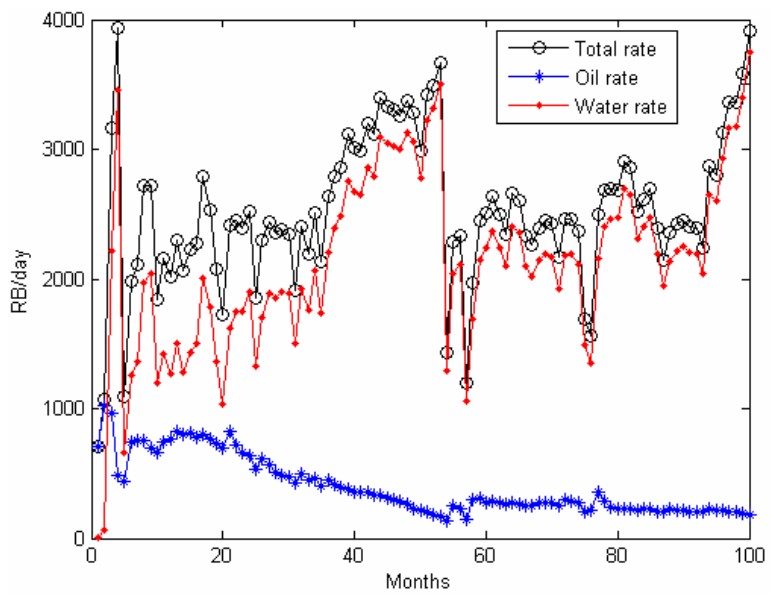

Figure 3a. Rates for producer P01

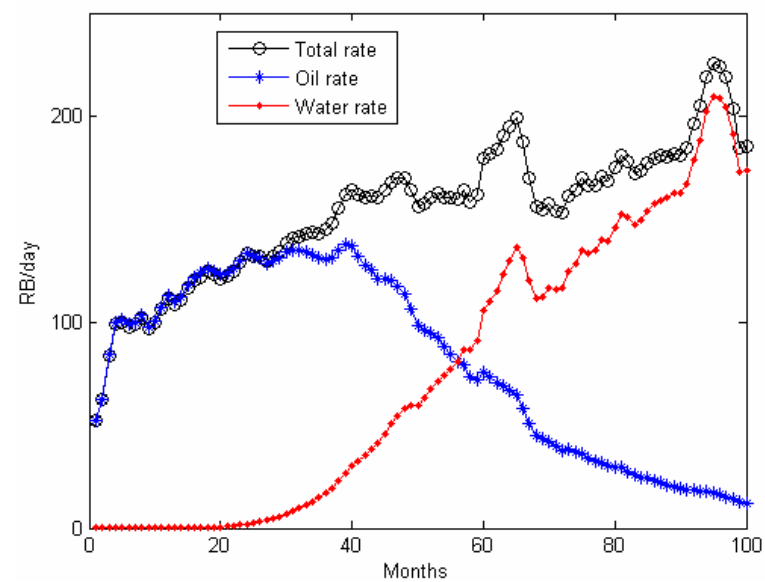

Figure 3b. Rates for producer P02

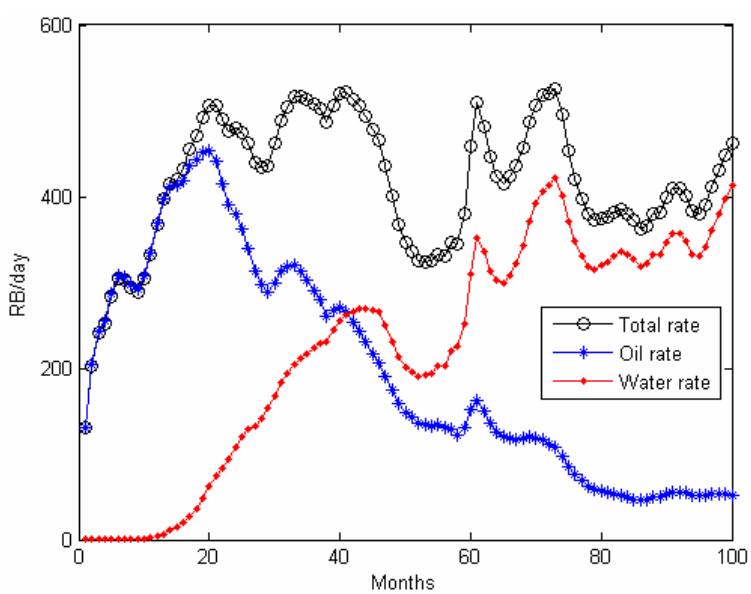

Figure 3c. Rates for producer P03 


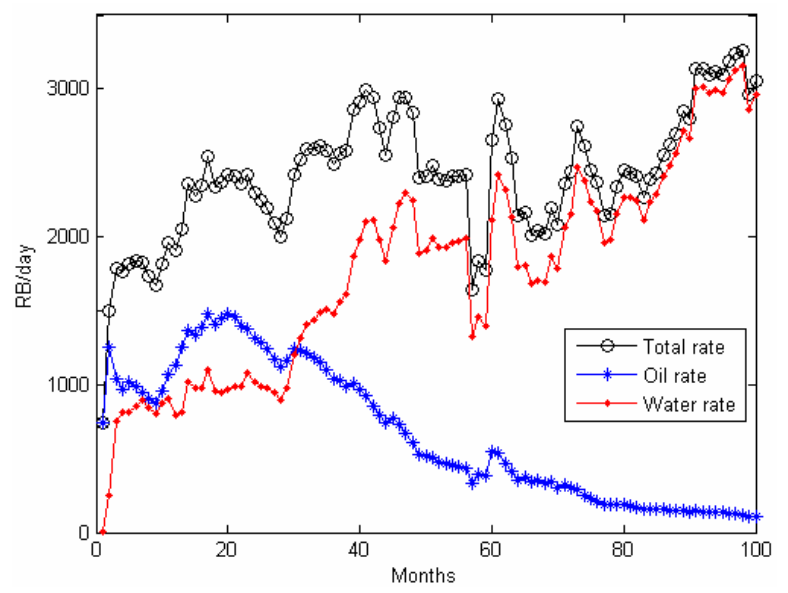

Figure 3d. Rates for producer P04

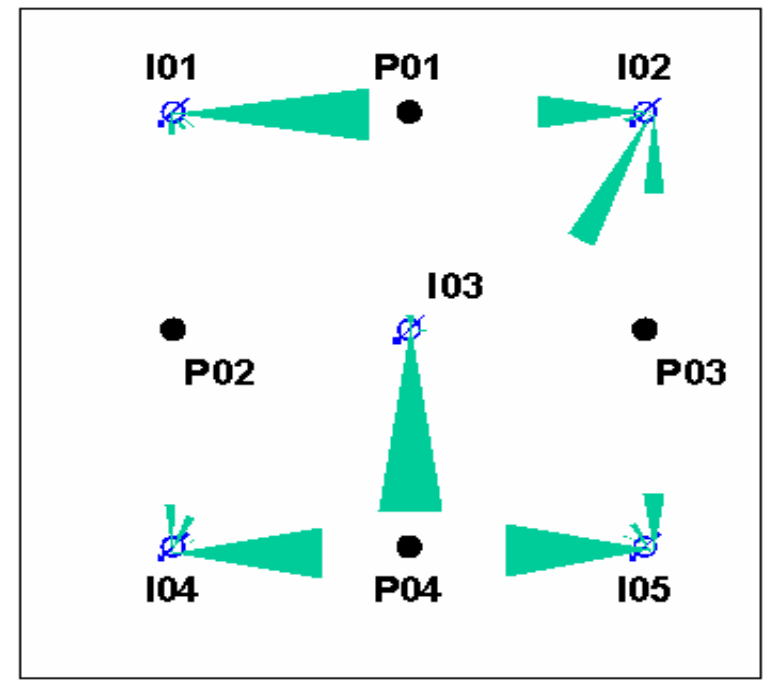

Figure 4. Representation of the Weights

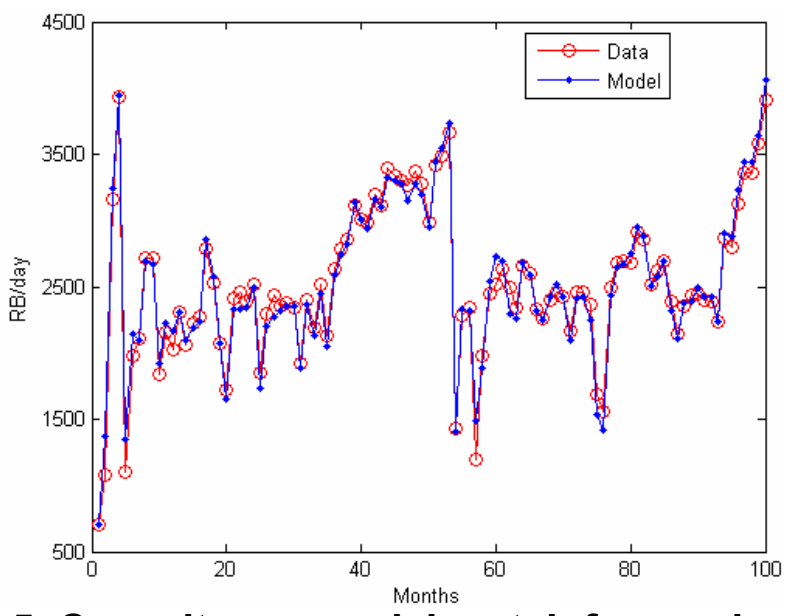

Figure 5. Capacitance model match for producer P01 


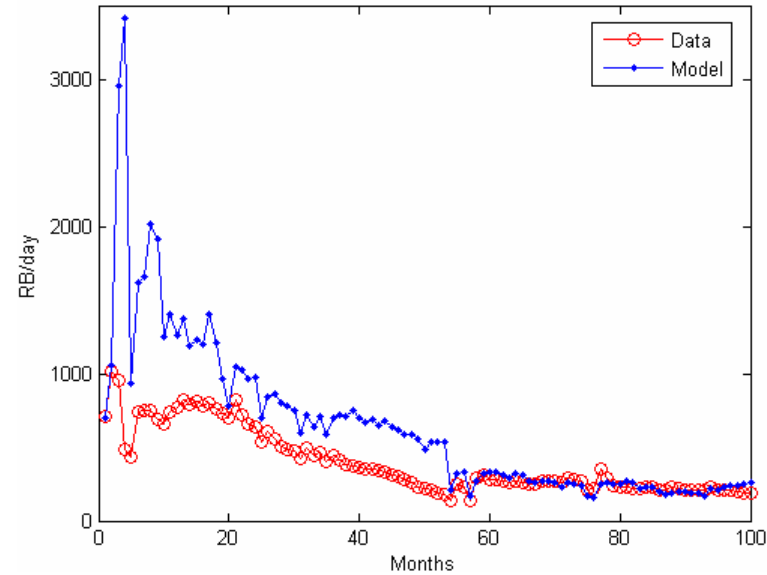

Figure 6a. Oil production model match for producer P01

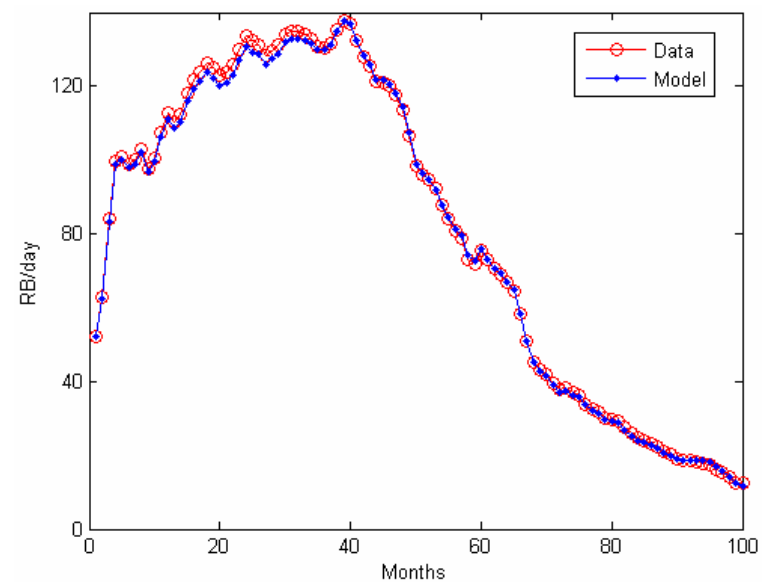

Figure $6 \mathrm{~b}$. Oil production model match for producer P02

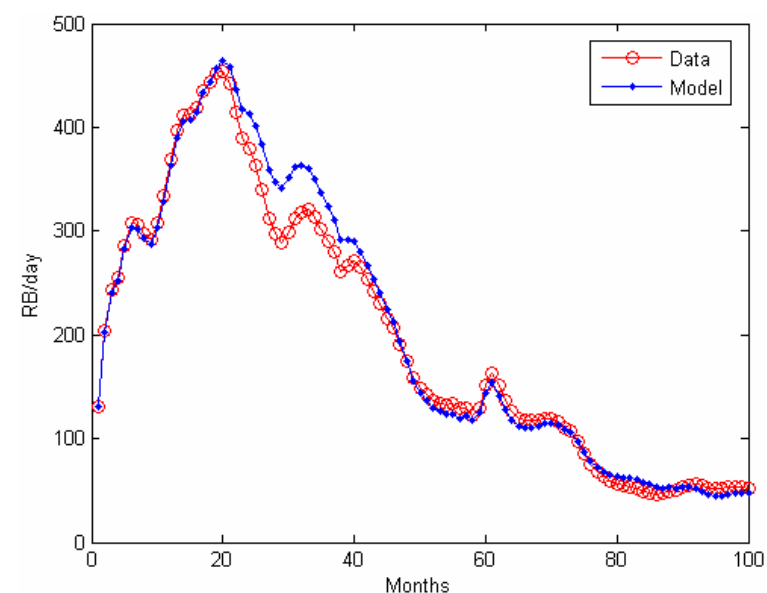

Figure 6c. Oil production model match for producer P03 


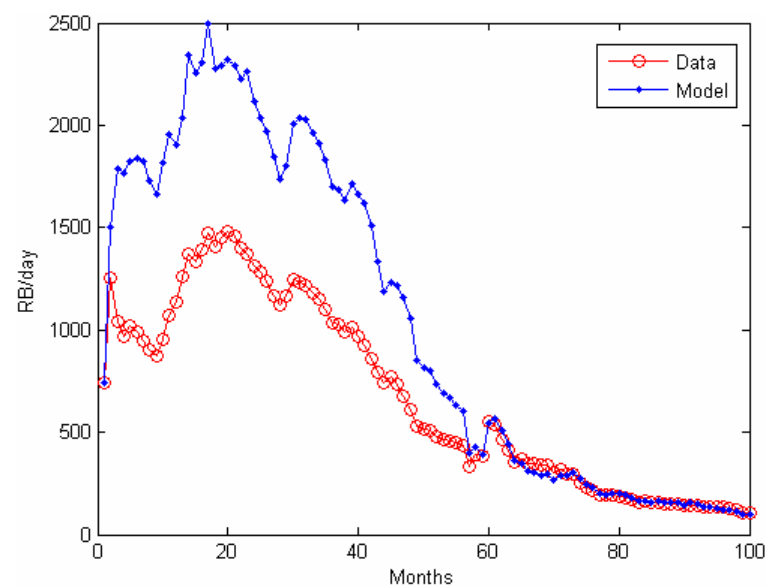

Figure 6d. Oil production model match for producer P04

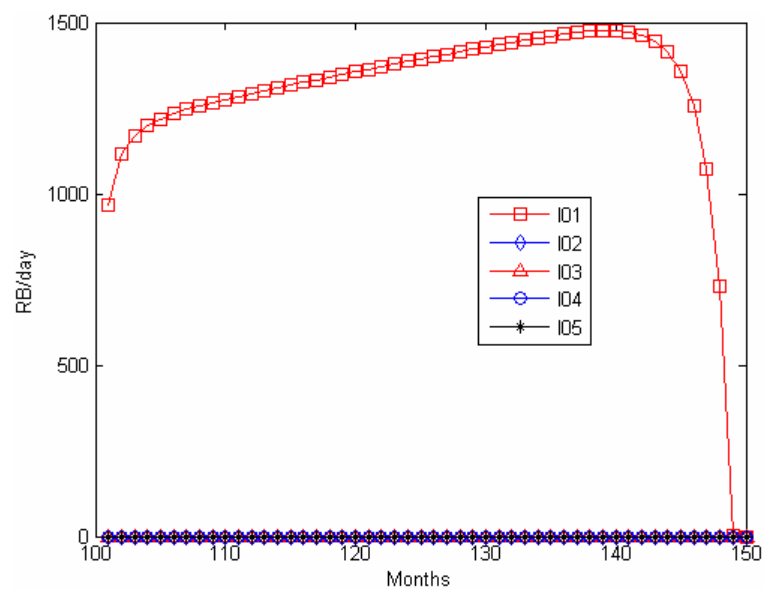

Figure 7a. Optimal injection rates at oil price $\$ 20 / R B$ and water injected price \$2/RB

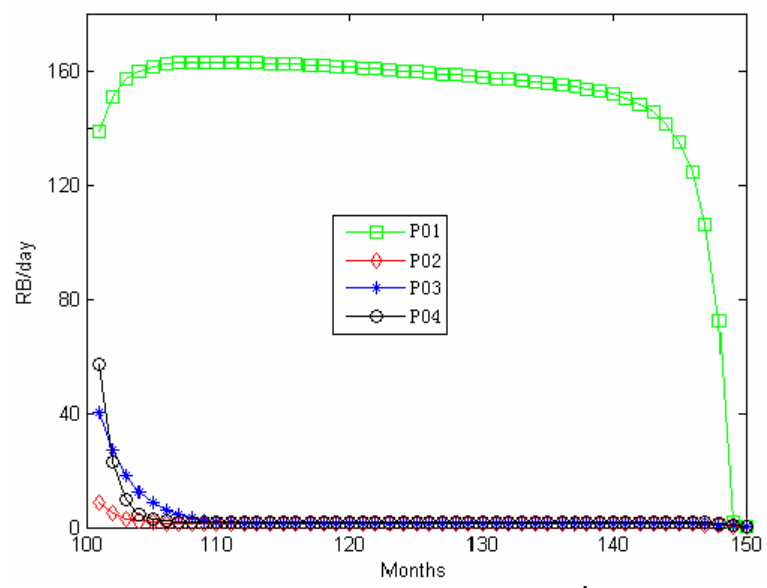

Figure $7 \mathrm{~b}$. Optimal oil production rates at oil price $\$ 20 /$ RB and water injected price \$2/RB 


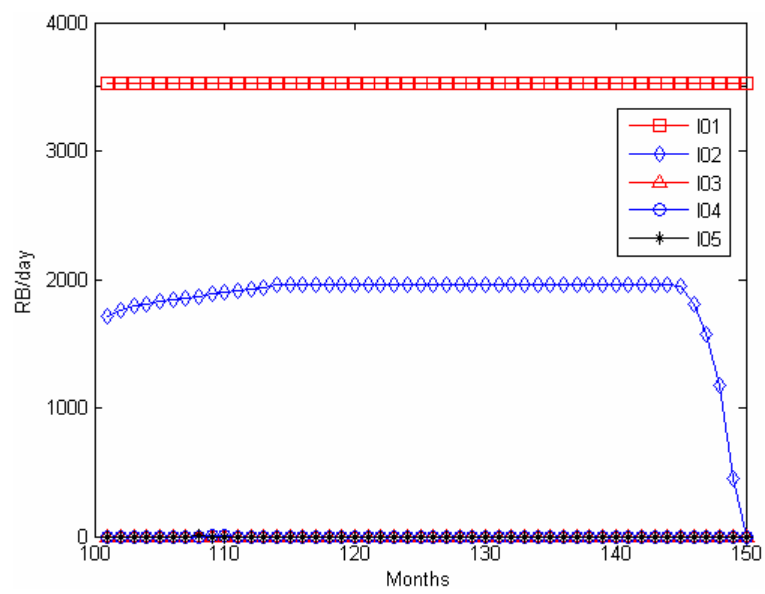

Figure 8a. Optimal injection rates at oil price $\$ 60 /$ RB and water injected price \$2/RB

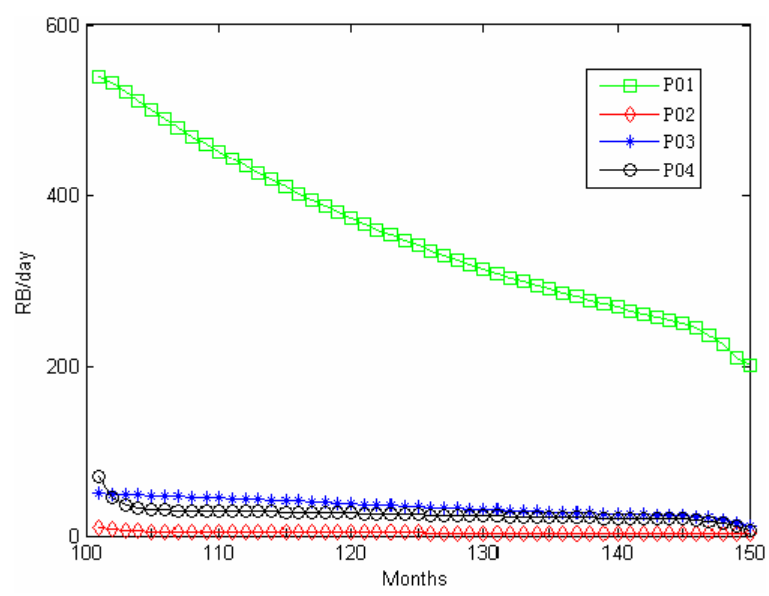

Figure 8b. Optimal oil production rates at oil price $\$ 60 /$ RB and water injected price \$2/RB

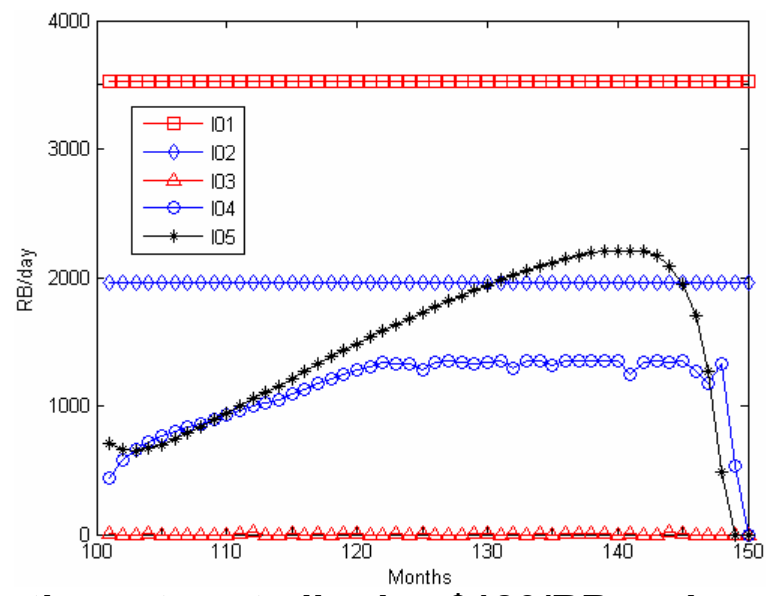

Figure 9a. Optimal injection rates at oil price $\$ 180 /$ RB and water injected price \$2/RB 


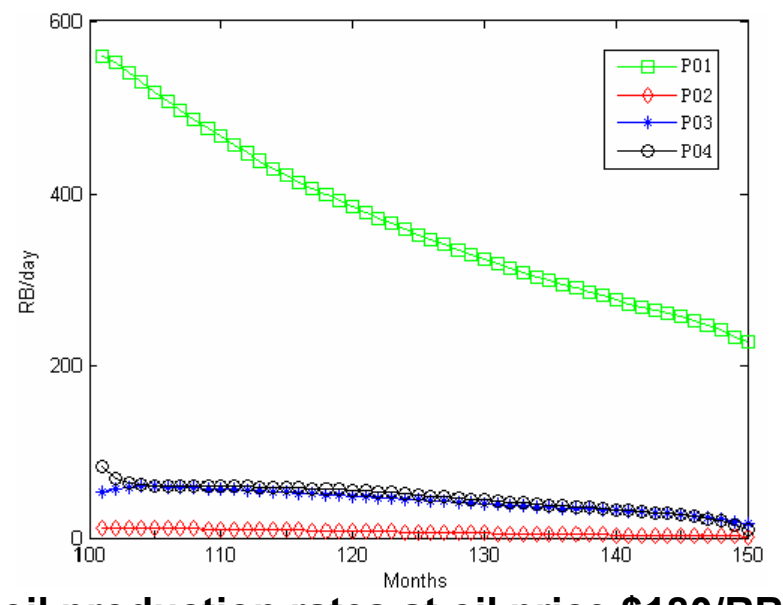

Figure 9b. Optimal oil production rates at oil price $\$ 180 /$ RB and water injected price $\$ 2 / R B$

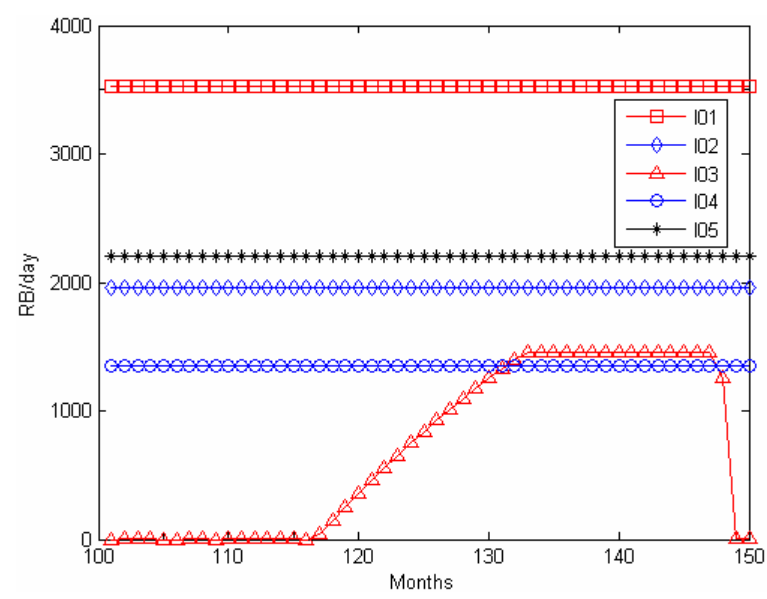

Figure 10a. Optimal injection rates at oil price $\$ 400 / R B$ and water injected price $\$ 2 / R B$

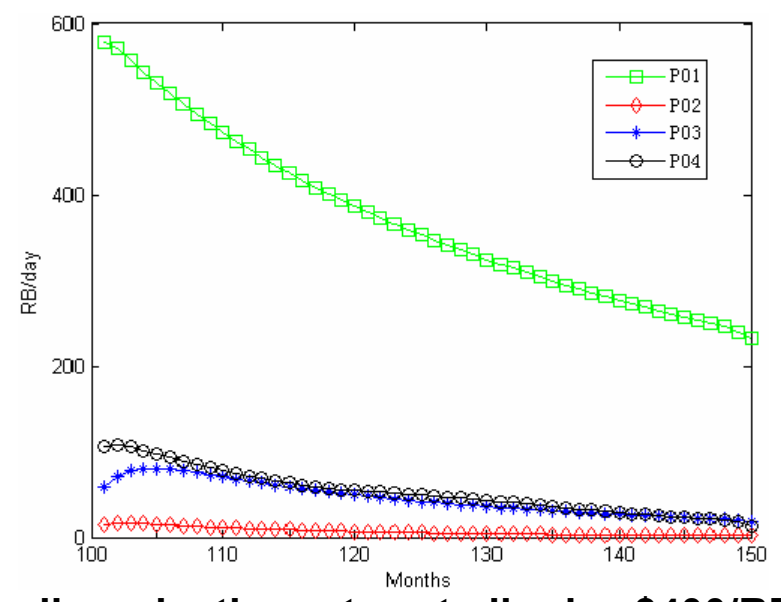

Figure 10b. Optimal oil production rates at oil price $\$ 400 / R B$ and water injected price $\$ 2 / R B$ 


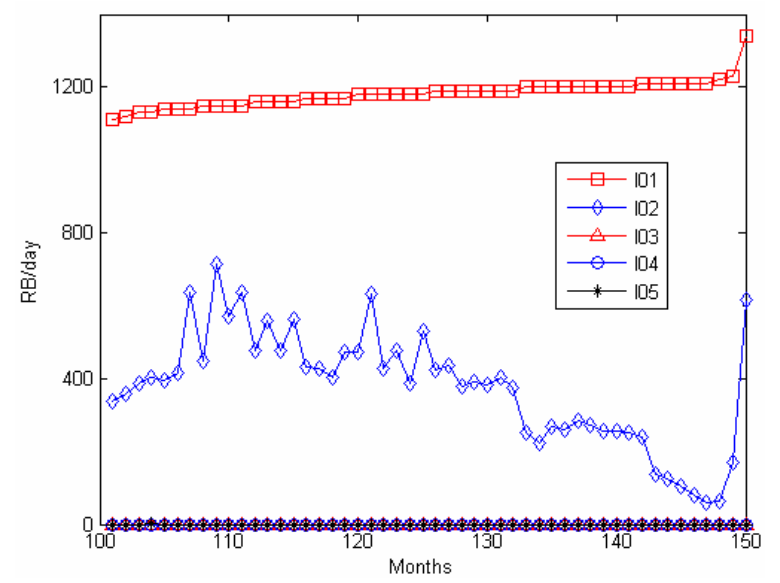

Figure 11a. Optimal injection rates at oil price $\$ 20 / R B$ and water disposed price $\$ 1 / R B$

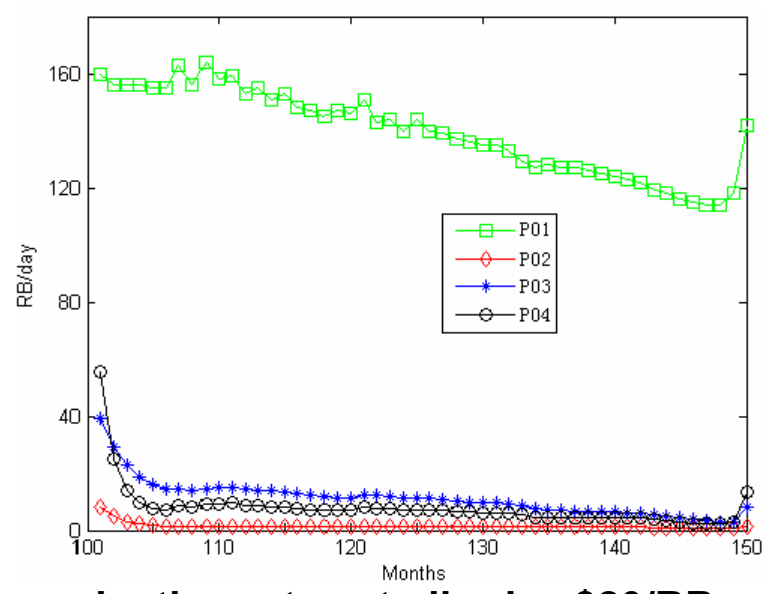

Figure 11b. Optimal oil production rates at oil price $\$ 20 / R B$ and water disposed price $\$ 1 / R B$

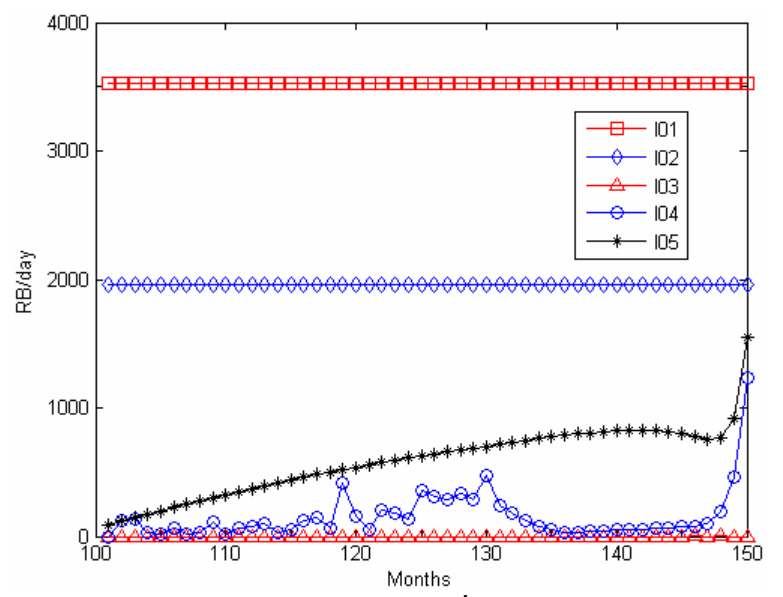

Figure 12a. Optimal injection rates at oil price $\$ 60 / \mathrm{RB}$ and water disposed price $\$ 1 / \mathrm{RB}$ 


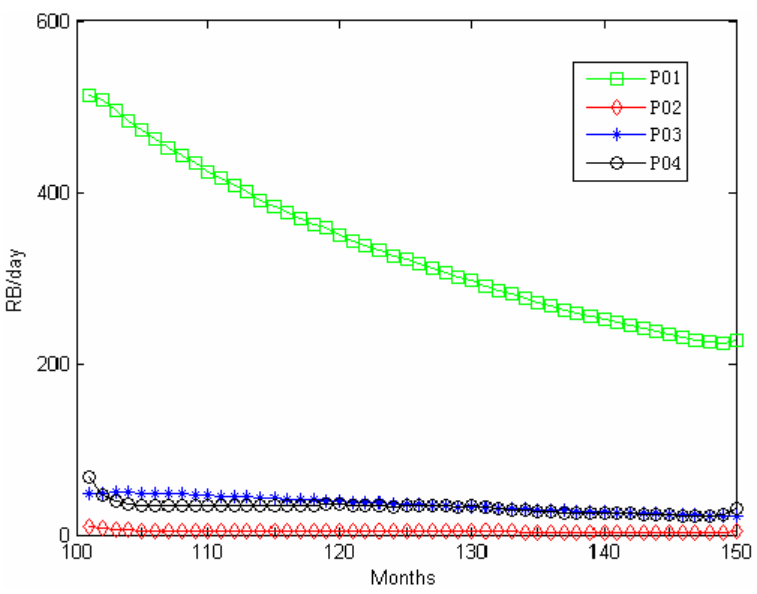

Figure 12b. Optimal oil production rates at oil price $\$ 60 / R B$ and water disposed price \$1/RB

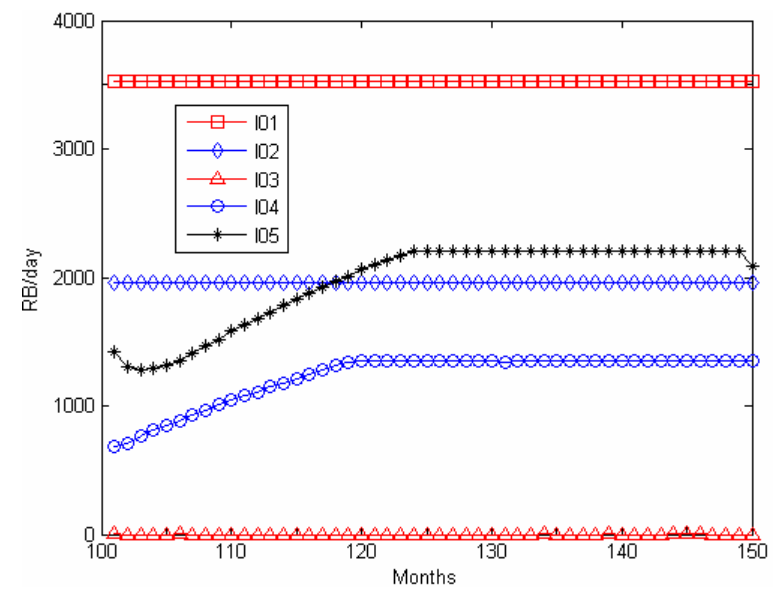

Figure 13a. Optimal injection rates at oil price \$120/RB and water disposed price \$1/RB

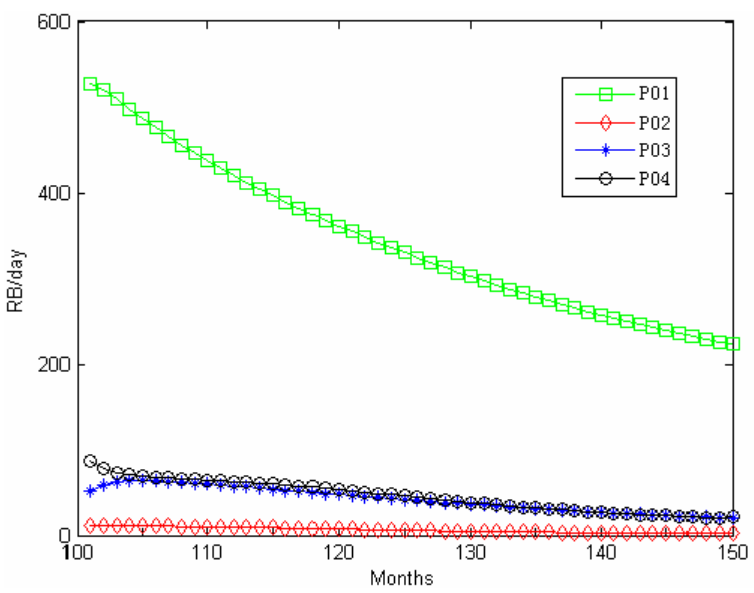

Figure 13b. Optimal oil production rates at oil price \$120/RB and water disposed price \$1/RB 


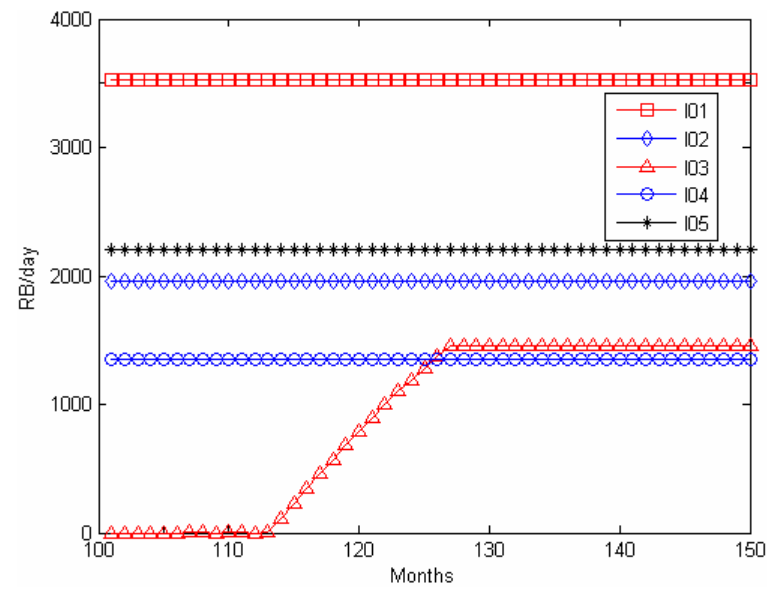

Figure 14a. Optimal injection rates at oil price \$240/RB and water disposed price \$1/RB

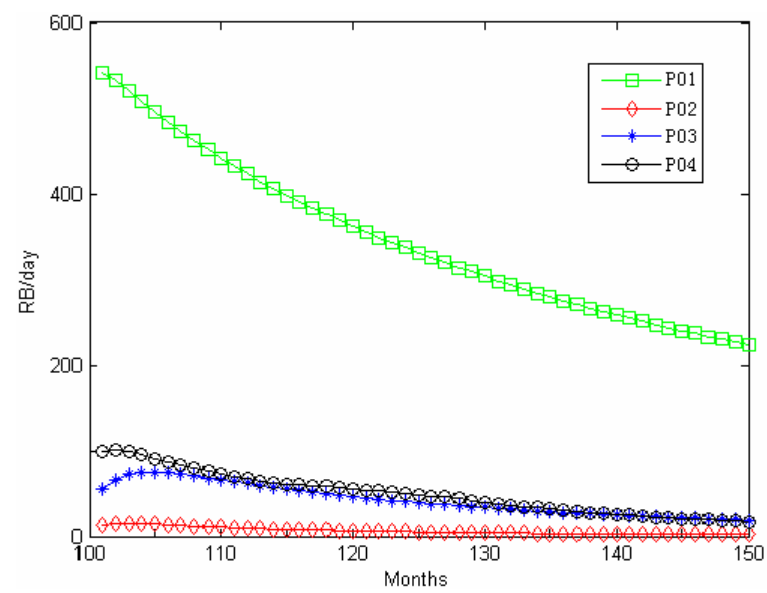

Figure 14b. Optimal oil production rates at oil price \$240/RB and water disposed price \$1/RB 


\section{RESULTS AND DISCUSSION \\ PART 3. SPECTRAL ANALYSIS OF INJECTION AND PRODUCTION DATA}

\subsection{Introduction}

In this part of the project, the reservoir continuity and geological complexity issues have been interpreted in a new method. It is a proof of principle that uses a frequency based representation of the injection and production flow rate data coupled with an analogous resistance-capacitance network model. This method enables us to infer the connectivity between well pairs and quantify the parameters of the diffusivity coefficient.

In theory, an analogy between the diffusivity equation and the transmission line equation that expresses the voltage distribution in an electrical circuit is used to identify the reservoir model. Identifying the model provides the corresponding diffusivity coefficient; hence inferring the interwell connectivity.

The Fourier transforms of flow rate data are used to represent them in their frequency spectra and analyze the response to impulse ratio which is named as the reservoir transfer function. Reservoir transfer functions are used to describe the reservoir model and build the analogy between RC models.

This study aims to investigate the free gas production around the wellbore, which has been excluded in the interwell connectivity studies.

\subsection{Literature Review}

\subsubsection{Interwell Connectivity Studies}

There have been various studies that have attempted to resolve the uncertainty in interwell connectivity and to quantify the degree of communication between wells in a reservoir. These methods include non-parametric statistics, wavelet analysis, neural network studies and the capacitance model that has been used in this project.

1. Heffer et al. (1995) developed a new method to test two main principles:

a. The rate correlations between wells are generally good indicators of communication in a reservoir.

b. The rate correlations between wells can be explained partially by geomechanical processes.

The method that they developed, couples the lateral direction of the fluid flow and the orientation of horizontal earth stresses. To predict the lateral communicaton between wells, they used the Spearman rank correlation coefficients between pairs of wells, each comprising injectors and producers. The Spearman rank correlation is a very convenient non-parametric (distribution-free) statistic which is used as a measure of the strength of the associations between the injector and producer flow rate data. However, the correlations that were calculated gave spurious negative correlations and no systematic behavior of correlations with time lag was found. Therefore, the applications were conducted with no time lag. Eventually, the two principles mentioned above were validated by the applications and it was concluded that the coupled model provides a better understanding of the reservoir behavior to optimize the waterflooding planning.

2. Refunjol (1996) applied the Spearman rank correlation coefficient to the flow rates of injector/producer pairs composed of each injection well and all adjacent producers. In addition to the current literature at that time, she investigated the effect of time lag between injectors and producers for the first time. Different time lags were used to find a maximum 
rank correlation coefficient at a time lag of maximum correlation for each possible injector production pair. So that, the maximum coefficient values of all injectors could be used to predict the preferential flow trend in the reservoir. The results of her study also provided negative correlation coefficients which were explained by the influence of a third well.

3. De Sant Anna'Pizarro (1999) also used the Spearman rank correlation coefficient between the well pairs. He aimed to test the validity of the non-parametric statistics method and to explain the reasons of the negative correlation coefficients that Refunjol ended up with. He used numerical simulations and pointed out the advantages and limitations of using the Spearman rank correlation coefficient of the flow rate data in reservoir characterization. $\mathrm{He}$ proved that the method seemed to be more appropriate to indicate the potential flow barriers and permeability anisotropy. He also concluded that Spearman rank correlation coefficient can increase if the injection rate is autocorrelated with time.

4. Jansen and Kelkar (1997) introduced the use of wavelet transform to describe the interwell relationships, stating that the conventional cross correlation techniques tend to fail since the production data itself are non-stationary. Thus, they suggested that production data needed to be treated firstly by wavelet transform, so that it could be broken down into its frequency spectra, then the cross correlation between the pairs of wells could be performed. Then, the interwell relationships could be described more reliably. The significance of this study was its ability to break the flow rate data into its frequency components and still remain the time information so that time dependent cross correlation methods could also be applied. They also believed that another property of the Wavelet transform, which is the ability of treating non-stationary data, played a very important role while capturing the dynamics of the well behavior better. For this reason, more reliable correlations between well pairs could be maintained.

5. Panda and Chopra (1998) suggested a different, integrated approach other than the statisticsdriven methods to estimate the well interactions. They introduced an artificial neural network application where the multivariate data set including not only the flow rate data of the well pairs, but the petrophysical parameters and well locations in a reservoir could also be considered in analyses. The data set was trained by a neural network and the results were used to determine the presence of reservoir heterogeneities and the permeability trends. Eventually, this method seemed to work more accurately rather than cross correlating the flow rate data visually, therefore it could be more successful in management of a waterflooding project. However, this method had some limitations such as the lack of physical process representation in neural networks. That limited its use in numerical simulation. The authors stated that it required more research.

6. Soeriawinata and Kelkar (1999) also applied Spearman rank cross correlation by considering that the well pairs operating simultaneously cause both superposition and noise. The authors stated that the superposition of injectors has significant effect on a producer's response which could be either constructive or destructive interference. The method includes a trial and error approach which aims to select the best well pair that has the highest correlation coefficient. The study was applied on a hypothetical waterflooding project as well as on a real field. The authors stated that the injected water flowing outside the lease boundary and the noise due to the inaccuracy in measurements resulted lower cross correlations in the real field case, as they expected. An important assumption was done during this study which was the cross correlation of the summation of the water injection rates to the liquid production rate would be higher than the cross correlation of each single injector. Eventually, it was noted that this 
method was very helpful to define the reservoir connectivity and easy to use for small operator and producer companies.

7. Albertoni and Lake (2003) introduced a technique to estimate the interwell connectivity based on a linear model by combining multivariate regression analysis with diffusivity filters that account for the time lags and the attenuation between the wells. The method enables to perform a fieldwide analysis where all the wells can be analyzed at a single step. It offers two solution approaches when the fieldwide injection rate is different than the fieldwide liquid production rate and when the two are almost equal. The linear model weighting coefficients are shown to be independent of the injection and production rate data and depend solely on geology and relative position amongst the wells. The model they used is a steady state and purely resistive model which is useful for determining the permeability trends and the presence of permeability barriers. The study is validated on two different hypothetical fields and validated with an application to a real field in Argentina. They tested the hypothetical models in anisotropic media through multi-layered reservoirs, reservoirs with a sealing fault or partially sealing faults or with a channel. The applications of this method were restricted to only oil and water production and free gas production was not included in the calculations. The assumptions that were made throughout the work are no change in the number of wells, in BHP of the producers, in skin and well properties, in total compressibility factor, completion procedures, the effective permeabilities and in primary production.

8. Al-Yousef et.al. (2005) improved the technique introduced by Albertoni and Lake (2003) to describe the interwell connectivity based on fluctuations in production and injection rates. He extended the work by using a nonlinear model which is not only a purely resistive model, but also a capacitance model that accounts for the compressibility and the time lags. Hence, the new model he developed does not require filtering process and performs better when the measured coefficients reflecting the connectivity are compared with the known geology and the locations of the wells in the real field. The model determines two coefficients to provide a better and more precise understanding about the interwell connectivity. The first of these two coefficients is the weight that quantifies the connectivity and depends on only the geology and the relative position of wells. The second coefficient is the time constant which quantifies the degree of fluid storage between wells and accounts for the attenuation and the time lag between the pairs of the wells. The advantages of this method compared to the previous work are the applicability to the fields where the wells are frequently shut-in or the primary production is remnant and also its ability to incorporate the BHP values if available.

\subsubsection{Studies using sinusoidal injection rates in reservoir description}

1. Johnson, et.al (1966) developed a new method named pulse-testing alternative to interference tests. The interference test is not practical due to the interruption of all field operations held during the test. Also, very long period of time is necessary to obtain a measurable pressure response with the conventional gauge at the responding well. The method they developed is able to measure transmissibility and storage between a pulsing well and a responding well with the help of a more sensitive gauge than the conventional one. The advantage of pulsetesting is that imposing a series of pulses (cyclic disturbances) makes it easier to diagnose and distinguish the pressure response from noise at the responding well. The exponential integral solution for the unsteady state flow model where a homogenous, single-phase and slightly compressible reservoir is assumed is used to describe the pulse-test pressure response. The response amplitude and the time lag obtained from the test results are used to calculate the average values for transmissibility and storage. In simple reservoirs where the assumptions of 
the analytical solution are met, this method can provide quantitative values for reservoir parameters; on the other hand it provides qualitative information about heterogeneous reservoirs. It is concluded that the response amplitude is dependent on well spacing, transmissibility, pulse interval and shut-in period as well as the rate of the pulse.

2. Kuo (1971) introduced analyses to determine reservoir properties from flow tests in one or more wells by imposing either sinusoidal or periodic constant multiple rates. He derived the pressure response equations based on the radial diffusivity equation and offered equations to calculate permeability values and the skin factor. In the case where a sinusoidal flow rate is imposed at either a single well or multi-wells, the pressure responses are calculated from the equations and the reservoir parameters are measured from the maximum response amplitude and the phase lag. On the other hand, in application of periodic multiple rates, formation parameters are calculated from the slope and intercept of a linear pressure response versus time function. The result of that work was that if a sinusoidal flow rate is imposed to a well, the responding flow rate will be also periodic after the transients die down. This method is very similar to the conventional drawdown or multi rate flow tests; however it differs in regulating the flow rates and interpreting the results. Those solutions are valid under homogenous, single-phase and slightly compressible reservoir conditions and have no advantages compared to the previous work by Johnson et.al (1966). However, in heterogeneous reservoirs plotting the amplitude ratio and phase shift versus frequency provides a possibility of determining heterogeneities. Moreover, application of both single and multi-well tests enables flexibility to compare the solutions between wells and offer more accurate results.

3. Rosa and Horne (1991) developed an analytical solution to quantify the pressure response caused by cyclic flow rate variations in a heterogeneous reservoir with radial permeability variation assuming single phase flow, slightly compressible fluid and isotropic porous medium properties. They applied the perturbation method for a qualitative analysis and to initiate the quantitative study. The method comes up with a useful formula that expresses the radius of cyclic influence in terms of the frequency of the cyclic flow rate. And this formula is used to design the pulse-test to yield the most optimum result in estimating the permeability distribution. The results of this work on multi composite radial reservoirs are very enlightening in terms of understanding the frequency dependent mechanisms in a reservoir. For instance, it is concluded that pressure responses caused by the flow disturbances are sensitive to the permeabilities of different regions. It is shown that higher the frequencies, larger the region of influence between the well pairs at multi-well flow tests. The idea behind that is the increasing frequency increases the contribution from the permeabilities near the pulsing well because the high frequencies do not deeply go into the reservoir, they just go to the region near the pulsing well.

4. Hollaender, et.al (2002) propose that harmonic testing can be used to determine the same reservoir parameters as well as conventional testing. However, there are some limitations and also some advantages in using flow rate data which are generated periodically during well tests. These issues are explained by several practical applications. In some applications, Fourier transform is used to determine the amplitude and the phase shift of the sinusoidal variation of the flow rate to use in analysis of the test. It is concluded that it takes more time to run harmonic testing to obtain the same amount of information in conventional testing. The good point is that it does not require to interrupt the production. These tests are less influenced by the noise and the potential noise can be averaged effectively. Since there is not 
a very high signal-to noise ratio, it enables to operate with small rate changes, which minimizes rate dependent non-Darcy effects.

\subsection{Issues Investigated}

\subsubsection{Method}

As noted by Muskat (1949), an electrical analyzer is a powerful tool to analyze water-drive reservoirs when the water reservoir itself is not of simple geometry or uniform physical properties. Keeping this in mind, the basic idea here is to assume that the reservoir system is equivalent to a resistance-capacitance network model, where the fluid flow in a porous media is equivalent to the flow of an electric current in a circuit (Fig. 1).

The starting point of the proposed theory is the application of the transmission line equations of an RC network model in describing the diffusivity coefficient. Eq. 1 is the fluid flow diffusivity equation in petroleum engineering studies (Lee, 2003) and Eq. 2 is the solution of the transmission line equation for the voltage distribution in communications engineering (Everitt, 1956). Imposing the same boundary conditions on these two equations ends up with the similar distributions in both pressure and voltage.

$$
\begin{aligned}
& \frac{\partial p^{2}}{\partial z^{2}}=\frac{\phi \mu c_{t}}{k} \frac{\partial p}{\partial t} \\
& \frac{\partial v^{2}}{\partial z^{2}}=R C \frac{\partial v}{\partial t}
\end{aligned}
$$

Comparing Eqs. 1 and 2, we observe the analogy between the RC network and the reservoir that we are to analyze. As shown by the analogy between these equations, the pressure difference in a reservoir between two locations is equivalent to the difference in voltage in an RC network between two terminals. Therefore, the analogy can be summarized in Eq.3:

$$
\frac{\phi \mu C_{t}}{k} \sim R C
$$

The units of both expressions in Eq. 3 are in $\mathrm{T} / \mathrm{L}^{2}$.

After explaining the link between RC networks and reservoir, it is important how to construct this link. In electrical engineering, it is a very common and simple procedure to apply a sinusoidal input to the RC network and to measure the system output at each frequency. The transfer function which is the output to input ratio can be calculated and plotted versus frequency. This plot can be used to represent the efficiency of the RC network as the frequency changes.

Likewise theoretically, this simple procedure can be applied to the reservoir assuming the injection rate data as the input and the production rate data as the output. However, in fluid flow it is not as simple as in electrical engineering practices to distinguish the frequency components of the rate profile. Therefore, Fourier transform is used to decompose the flow rate into its harmonic components, thus it can be expressed in terms of its frequencies. This way, it is possible to calculate the transfer function of a reservoir and plot it in the frequency spectrum as well as RC network transfer functions.

In theory, the procedure should be working perfectly well, however there are some sources of error that masks the reservoir transfer function spectra. These are

- the noise of the production data caused by the numerical simulation.

- high compressible reservoirs

- the reservoir that has a large drainage area 
- undersampling the data that causes aliasing(sampling once per day is proven that it does not cause aliasing in our case, however low frequency components might still be dumped into the high frequencies)

These issues will be addressed later more in the conclusion section.

Fourier analysis is named after Jean Baptiste Fourier, who showed that any periodic function can be represented as the sum of sinusoids with integrally related frequencies. The purpose of these analyses is to express a given arbitrary function, as a linear combination of a set of harmonic sine and cosine basis functions (Harman, 2000).

$$
f(t) \approx \frac{a_{0}}{2}+\sum_{n=1}^{N}\left[a_{n} \operatorname{Cos}(n t)+b_{n} \operatorname{Sin}(n t)\right]=s_{N}(t)
$$

where $s_{N}(t)$ is the Fourier approximation to the function $f(t)$ by using a trigonometric polynomial of degree $N$ assuming $f(t)$ is continuous on the interval $[-\pi, \pi]$. The constant term $a_{0}$ and the coefficients $a_{n}$ and $b_{n}$ in Eq. 4 can be computed by the following formulas:

$a_{0}=\frac{1}{\pi} \int_{-\pi}^{\pi} f(t) d t$

$a_{n}=\frac{1}{\pi} \int_{-\pi}^{\pi} f(t) \cos (n t) d t$

$b_{n}=\frac{1}{\pi} \int_{-\pi}^{\pi} f(t) \sin (n t) d t$

In Fig. 2, a raw signal and the spectrum of that signal are shown. In Fig. 2-a, given raw signal, $\mathrm{f}(\mathrm{t})$ in time domain is defined as the following equation $\operatorname{Cos}(2 \mathrm{pi}(0.125 \mathrm{t})+\operatorname{Cos}(2 \mathrm{pi}(0.05 \mathrm{t}))$. In Fig. 2-b, the Fourier transform of the raw signal is represented. The frequency components of that signal in the spectrum are $0.05 \mathrm{~Hz}$ and $0.125 \mathrm{~Hz}$, respectively. The purpose of these figures is to prove that Fourier transform is applied to obtain further information from the signal that is not already available in the raw signal. The further information is actually hidden in the frequency content of the signal. This basically explains why we use the Fourier transform of the flow rate data.

In this study, Fourier transforms of the flow rate data are calculated in Matlab using the fast Fourier transform algorithm. By this method, it is aimed that the dominant periodic components of a reservoir can be discerned to be able to identify the reservoir behavior.

To be able to identify the reservoir behavior, three analytical RC network models are calculated. These models represent three solutions given different boundary conditions to Eq. 2:

- 1-D Model: This is the solution to a 1-D voltage distribution in an RC network. It is analogous to the fluid flow in a very narrow reservoir where flow is restricted only in one direction. This may be a channel, a fracture.

- 2-D Model: This is the solution to a 2-D voltage distribution. It is analogous to the fluid flow in a very large reservoir where drainage area is large enough for a multi-dimensional flow.

- Lumped (0-D) Model: This is the solution of a transmission line equation where all dimensions in a RC network are coupled and represented in only one dimension which is 0 dimensions. An example of RC models that are calculated for the given reservoir parameters are represented in Fig. 3.

The analogy between RC network and reservoir is constructed because the solutions to both Eq.1 and Eq. 2 would give the similar result as long as their boundary conditions are imposed the 
same. Moreover, calculating the same coefficients in Eq. 3 is expected to give the same distributions in pressure and in voltage. Therefore, this work aims to compare the reservoir and the voltage transfer functions with each other on the same plot and to select the best RC model that captures the behavior of the reservoir, visually for now. A quantitative approach to select $\mathrm{RC}$ models will be introduced later. Picking the RC model that captures the reservoir behavior best will let us construct the analogy between the RC model and the reservoir. Thus, the data obtained from the $\mathrm{RC}$ given the reservoir properties will yield us the interwell connectivity in terms of $\phi / \mathrm{k}$ as it is given by Eq. 3 .

\subsubsection{Choice of injection rates}

Contrary to using a sinusoidal injection rate that has particular frequencies in it, using random injection rate that contains all frequency components is more useful and better to determine the reservoir behavior. As noted earlier, the Fourier transform is used specifically to emphasize the dominance of the periodic components in a signal. In this work, the characteristics of low and high frequency components are more important to identify rather than only one specific frequency component coming from an injection process. This is because the reservoir behavior and the well interferences are characterized by both low and high frequency components.

The theoretical basis of using a random time series is that the frequency response of an infinitely long random time series at each frequency should be 1, since the time series contain all the frequencies. Fig. 4 is plotted with using 10,000 points to create a long enough time series to approach this theory. The Fourier transform of that time series which is a random generated normal distribution, is calculated and the Fourier spectrum is plotted on graph.

Fig. 5a and $\mathbf{5 b}$ are shown to explain the difference better between the Fourier spectra of a random time series and a cyclic time series. As it is seen in Fig. 5a, the frequency components of the time series at every 100 days are seen at low frequencies. As the frequency increases, the dominance of high frequencies is discerned and it gets tougher to distinguish the components from one another. Unlike in Fig. 5b, since there is no dominancy amongst frequencies in the data, any change in the producer will be discerned very easily in comparison to this spectrum. Therefore, such data forms a very good and reliable basis to start identifying the behavior of the reservoir.

The resolution issue of the spectra is also addressed by comparing Fig. 4 and Fig. 5b. The more points used in the analysis, the better the resolution will be obtained. Considering the computer simulation run time, the resolution criterion and the fast Fourier algorithm which requires the number of points to be calculated to be a power of 2, the resultant number of points used in the analysis is determined to be 4096 . Hence, the time series will be used as the injection rate profile in further study is shown in Fig. 6. It is a random normal distribution with a mean value of 1000 and a standard deviation of 200 .

\subsubsection{Simulation model validation}

In this part, there is an illustration of a sensitivity analysis apart from the scope of this work. This part is necessary in obtaining reliable answers from the numerical reservoir simulations. It should be noted that the instability problem mentioned here does not appear in one phase production case. It is an issue to be discussed in two phase production as is explained here.

The reservoir model is a 1-D Cartesian model where there is one injector and one producer which are $1508 \mathrm{ft}$ apart. Water flooding is performed. The dead oil and water are produced at 250 psi.

The criterion to test whether the results are stable is to compare the production rates of the producer from two distinct runs: 
1. the case when there is no support from the injector. There is only primary depletion. (Qin_off)

2. the case when well is supported by the injector.(Qin_on)

The production rates when the injector is on should be higher than the case when the injector is off. However, the results will show that this is not the case for the early times of the simulation. Therefore, very fine gridblock and timestep refinements are needed to be used to overcome this problem, as it will be proved in the following paragraphs.

The reservoir geometry and the parameters used in the simulation model are shown in Table 1. Table 2 shows the gridblock and timestep refinements that were used for the sensitivity analyses. The reservoir geometry is shown in Fig. 7. It is important to mention that the fully implicit solution option in ECLIPSE is used throughout the runs.

The results of the sensitivity analyses represented in Figs. 8 and 9 show that the problem does not occur at favorable mobility ratio at all whereas at unfavorable mobility ratio the problem is seen at the very early life of the reservoir up to 2 days. It should be noted that these are the results at conditions where fine timesteps and slightly compressible fluids are used. The problem amplifies when the bigger timesteps and more compressible fluids are used. In Fig. 10, the results of the finest timestep ( 0.01 day) and the finest grid discretization (150x50x5) used in this study are illustrated. This means the best solution to the current instability problem is supposed to be solved under these conditions. However, in Fig. 10a and 10b, at very high compressibility values (1e-4 and 5e-5 1/psi) problem seems to be still an issue.

As a result of this sensitivity practice, it is shown that converging to 0 at both timestep discretization and grid discretization gives the best answer. However, it takes very long to run one simulation at the finest possible situation. Therefore, $150 \times 50 \times 5$ grid refinement and timestep at 0.1 days for further spectral analyses research, is performed.

\subsection{Spectral analysis}

The scope of this work is to calculate the reservoir transfer functions and compare them with RC network transfer functions which are calculated at three different boundary conditions. Subsequently, next step is to select the best RC network that captures the reservoir behavior. In spectral analysis, it is very vital to accurately calculate the reservoir transfer function which will represent the behavior of the reservoir. However, as noted in part 3.1, there are some sources of error that masks the spectrum and don't let the frequency components reveal the actual behavior of the reservoir. Therefore, a simple filtering process is needed at this stage. The spectrum is the frequency domain where the data is transformed into by fast Fourier algorithm and represented by its frequency components.

\subsubsection{Filtering process}

Filtering is applied over the random injection rate to smooth the noise and separate the signals in the data. The filter applied over the data is expected to be smoothing the noise in time domain, thus make it easier to separate the signals in the frequency domain. Most of the filters that do very good job in time domain perform very badly in the frequency domain. The moving average filter is an example of such filters.

Here it is important to explain what frequency response is. The frequency response of an impulse is obtained by taking the Fourier transform of the impulse and it tells how the information represented in frequency domain is being changed.

In this study, high frequency components (noise) are desired to be attenuated; therefore a well performing low-pass filter should be used. In theory, low-pass filters let the low frequencies pass 
through the filter whereas they block the passage of the high frequencies after a cutoff frequency. In practice, it is not possible to block high frequencies, but they can be attenuated up to a certain degree.

A low-pass filter is determined whether it performs well in the frequency domain by checking 3 parameters in its frequency response:

1. Fast transition from passband to stopband (cut-off frequency): This will separate the signals that are very close to each other.

2. Smooth passband: This is needed because low frequencies should not change while passing through the filter.

3. Good stopband attenuation: This will provide a good block to the high frequency components and will not let them interfere with the other frequencies.

In this work, Blackman window filter is applied over the data due to its good performance in the above mentioned parameters. Blackman window function is given in Eq. 7:

$$
w(i)=0.42-0.5 \operatorname{Cos}\left(\frac{2 \pi j}{N-1}\right)+0.08 \operatorname{Cos}\left(\frac{4 \pi j}{N-1}\right)
$$

Where

$\mathrm{N}=$ total number of points used in the curve.

Blackman window filter is classified in FIR(finite impulse response) type digital filters in digital signal processing terminology (Smith, 1997). And it requires convolution.

Filtering is applied over the injection rate time series before simulating it through the reservoir. The procedure is de-averaging the injection rate profile in Fig. 6, then convolving it with a normalized 4 point-Blackman window. After convolving the data, the previous data is named the raw data and the convolved output is called the filtered data (Fig. 11a). These two data sets now are transformed into frequency domain by FFT (Fig. 11b) and the transfer function is calculated:

$$
H_{1}(f)=\frac{Y(f)}{X(f)}
$$

Where

$Y(f)=$ Fourier transform of the filtered injection rate (convolved output)

$\mathrm{X}(\mathrm{f})=$ Fourier transform of the raw injection rate (rate data in Fig. 6)

$\mathrm{H}_{1}(\mathrm{f})=$ Transfer function of the filtering process

This transfer function yields a low-pass filter (Fig. 12). On this figure, it is possible to show all the 3 parameters that are mentioned above which are required to define a good low-pass filter. There is a very smooth pass band, the transition between passband and stopband is very fast(cutoff frequency $=0.021 /$ day) and stopband attenuation is very close to 0 with 3 orders of magnitude drop in the amplitude.

The summary of the filtering and the simulation procedures can be explained in the following schematic (Fig. 13). Basically, in the whole application there will be two important processes:

1. the convolution of the raw time series with Blackman window which provides a low-pass filter.

2. the simulation of the filtered time series through a particular reservoir model.

Final product in the schematic which is the simulated production rate is analyzed in its spectrum with respect to the injection rate spectrum. Then, the spectral analysis continues with building the transfer function of the reservoir (Eq. 9):

$H_{2}(f)=\frac{Z(f)}{Y(f)}$ 
Where

$Z(f)=$ Fourier transform of the simulated production rate

$\mathrm{Y}(\mathrm{f})=$ Fourier transform of the filtered injection rate (convolved output)

$\mathrm{H}_{2}(\mathrm{f})=$ Transfer function of the reservoir

Reservoir transfer functions are the key points which provide information about the reservoir behavior between the well pairs in terms of high and low frequencies. On the other hand, comparing them with analytical RC model solutions let us determine the reservoir model whether it is 1-D, 2-D or 0-D. determination of the reservoir model yields the corresponding diffusivity coefficient, thus the ranges of the reservoir parameters can be also identified.

\subsection{Results of Spectral Analysis}

Different than the previous part where sensitivity analyses are performed, only water phase is produced in applications of the method. The filtered injection rates (Fig. 11a) which are described in part 4.1 are used to simulate the production rates. Reservoir geometry and the parameters that are numerically simulated are tabulated in Table 3. The reservoir model is a 2-D Cartesian model where there is one injector and one producer which are $1508 \mathrm{ft}$ apart (Fig. 7). Waterflooding is performed. The following displayed results will vary due to the water compressibility factor used in the simulation. Other than that, all the reservoir properties and the injection rates remain the same.

\subsubsection{Cw=1e-6 1/psi}

In Fig. 14a, there are 3 sets of spectrum. These spectra stand for raw injection, filtered injection and the simulated production rates, respectively. As noted earlier, the objective of filtering the injection data is to create a low-pass filter. In other words, the objective is to block the high frequencies and not let them exist. Practically, this does not happen, what happens is the attenuation of these high frequencies in the stopband. In the figure, high frequencies in filtered injection rate spectrum begin to be attenuated at 0.11 /day; however it is obvious that the attenuation does not occur here very effectively. The reason is that the low fluid compressibility factor is so low that all the injected water is being produced without attenuating the high frequencies. In this case, reservoir does not do its filtering job very well because the high frequencies are able to travel through the reservoir and be produced at another well. In Fig. 14b, there is the reservoir transfer function and the corresponding $\mathrm{RC}$ models which are calculated given the reservoir parameters. First of all, it should be understood that this low compressibility case is not a good example of a low-pass filter. One of the limitations of this method is that it is less likely to distinguish the RC models from one another when small diffusivity coefficients are used in the analytical solutions. Even in the scarcity of information, lumped RC model seems to be capturing the reservoir behavior the best amongst all RC models. The lumped RC model reveals the frequency where transition between passband and stopband begins even though it is very high ( 0.21 /day). And it is obvious that the stopband does not even exist, only passband and some transition region are available at the reservoir transfer function.

The pink region that will be addressed in part 5.4, seems to be the result of applying two filtering process over the data but it needs more research. It is effective in this figure between frequencies $0.4-0.51 /$ day.

\subsubsection{Cw $=1 \mathrm{e}-5 \mathrm{1} / \mathrm{psi}$}

In Fig. 15a, there are the same 3 sets of spectrum. The difference in this case is that the simulated production rate spectrum is attenuated more than the previous case in Fig.13a because the fluid 
compressibility factor is more effective in the reservoir. Therefore, it attenuates the high frequencies of the pressure distribution and so the production rate is attenuated as well. In this case, it is more visible that the reservoir is doing a filtering job as well as the Blackman window filter. It is even a bigger filter because the frequency where the transition between passband and stopband is at lower frequencies $(0.031$ /day) in production spectrum than the one in filtered injection spectrum which is 0.1 1/day. This naturally yields more attenuation with 2 orders of magnitude drop in amplitude.

In Fig. 15b, the reservoir transfer function and the RC models are seen. In this higher diffusivity coefficient case, it is better to distinguish the differences between models visually. In this case, lumped model and the 2-D models are the ones that might be the answer to the question. However, lumped RC model is the one that fits the reservoir transfer function because it meets the requirements of a low-pass filter. If the transition frequency between passband and stopband is a characteristic of a low-pass filter, then lumped model which fits that frequency in this figure should be the one to be selected to define the interwell connectivity. 2-D model seems to be very poor in reflecting the reservoir behavior because it is uncertain where the transition begins and it does not also fit the lower end of the cloud unlike the lumped model. Obviously, interpretation in selecting the best RC model needs more quantitative approach, therefore future work will also include that.

The pink region (between $0.3-0.51$ day) effect amplifies in this case as the dominance of reservoir filtering job increases due to the compressibility factor.

\subsubsection{Cw $=1 \mathrm{e}-4$ 1/psi}

In Fig.16a, there is a representation of an extreme case where compressibility is very high (like a gas reservoir). Therefore, the reservoir filtering power is very dominant which yields very effective blockage to the high frequency components. The frequency between passband and stopband is at very low values ( $\sim 0.011 /$ day) and the stopband starts at frequency of $0.21 /$ day. This figure is a good representation of a low-pass filter including all the parameters needed in a filter, however the noise and potential sources of error caused by the numerical simulation are dominant in the spectrum. In Fig. 16b, the reservoir transfer function is seen. Amongst the RC models, 2-D model gives the best fit. However, the amount of noise and the width of the pink region play a great role here masking most of the necessary information. Therefore, identifying the reason of the pink region (between $0.1-0.51 /$ day) is very essential here.

\subsubsection{The source of error that forms at high frequencies}

The region defined in Figs. 14b-16b where the reservoir transfer functions move up at the very high frequencies despite the fact that they are supposed to bend down as in a low-pass filter, seems to occur when there are 2 filters applied over a data set. This evaluation is based on an analytical solution shown in Fig. 17. This figure is plotted by applying two Blackman window filters consecutively on the same raw injection rate data used in the spectral analysis. The first filter is a 4-point Blackman window which is the usual filter used in the previous applications. The second filter is another Blackman window either equal to 4 - point filter or greater than $4-$ point filter which has 4 different values in the figure: 4, 6, 8 and 10. This second filter is used instead of a reservoir simulation.

The analytical solution reveals that the problem does not occur as a result of reservoir simulation. It actually happens after applying two filters over a data set. The key point is the sequence of the filters. As it is seen in the graph, the solution where both of the filters are 4 - point filters, the 
problem does not occur. It occurs whenever the size of the second filter exceeds the previous filter. And the size of the error amplifies as the size of the second filter gets bigger.

The reason of this error is the division in transfer function calculation. Since the attenuation in the second filter is more than the previous one applied, the division operation does not give reasonable numbers after the point that the attenuation in second filter gets higher than the previous one. Therefore, this region should be ignored in the evaluation of the RC models. One thing that can be done to overcome this error would be to use bigger size filters at the first filter. However, this is not feasible to do since using a filter bigger than the reservoir would deviate from its objective of our study.

Eventually, reasoning of that region still needs to be evaluated with more analytical solutions and research.

\subsection{Conclusions}

1. The RC model that fit the reservoir transfer functions is the lumped RC model. Lumped RC model as mentioned earlier is the analytical solution of a transmission line equation where all dimensions in a $\mathrm{RC}$ network are coupled and represented in only one dimension which is 0 dimensions.

2. Reservoir models other than Fig. 7 are also used in this study. In those models, while keeping the interwell distance the constant, the reservoir geometry is changed. A large reservoir that has the dimensions of $1500 \mathrm{ft} \times 1500 \mathrm{ft} \times 20 \mathrm{ft}$ and a narrow reservoir with dimensions of $1500 \mathrm{ft} \times 75 \mathrm{ft} \times 20 \mathrm{ft}$ are used. The resultant RC model from these models is also lumped RC model.

3. Determining which RC model represents best the reservoir will lead the work to define the porosity - permeability relationships in the reservoir.

4. A quantitative approach needs to be used rather than a visual approach to interpret the reservoir transfer functions which result in a spread of amplitude as the frequency changes. The origin of those spread needs to be addressed.

5. The most important thing that can be withdrawn from the results of those applications is to establish the limitations of this study. These are

- the reservoir noise and the numerical simulation errors causing a noisy spectrum to evaluate

- $\quad$ high diffusivity coefficients are limiting the diagnose of the analogous RC models

- the sequence of 2 filters applied might be causing errors in high frequencies

- undersampling might be the reason of dumped amplitudes in high frequencies

- the reservoirs that have very large drainage areas limits the application

- the method is applicable in homogenous, single phase, isotropic porous media. 


\subsection{TABLES AND FIGURES}

Table 1 - Simulation model and reservoir parameters.

Testing the reservoir model

Base Case

\begin{tabular}{|c|c|}
\hline Reservoir dimensions & $1500 \times 150 \times 20 \mathrm{ft3}$ \\
\hline Interwell distance & $1508 \mathrm{ft}$ \\
\hline Production phase & water, dead oil \\
\hline$\phi$ & 0.1 d.less \\
\hline $\mathbf{k}$ & 100 md \\
\hline$\mu w$ & $1 \mathrm{cp}$ \\
\hline Bw & $1.01 \mathrm{RB} / \mathrm{STB}$ \\
\hline \multirow[t]{3}{*}{$\mathrm{Cw}$} & $1.00 \mathrm{E}-04$ 1/psi \\
\hline & $1.00 \mathrm{E}-05 \mathrm{1} / \mathrm{psi}$ \\
\hline & $1.00 \mathrm{E}-06 \mathrm{1} / \mathrm{psi}$ \\
\hline$\mu 0$ & $1 \mathrm{cp}$ \\
\hline Bo & $1.07 \mathrm{RB} / \mathrm{STB}$ \\
\hline \multirow[t]{3}{*}{ Co } & $1.00 \mathrm{E}-04 \mathrm{1} / \mathrm{psi}$ \\
\hline & $1.00 \mathrm{E}-05 \mathrm{1} / \mathrm{psi}$ \\
\hline & $1.00 \mathrm{E}-06 \mathrm{1} / \mathrm{psi}$ \\
\hline Cf & $1.00 \mathrm{E}-08 \mathrm{1} / \mathrm{psi}$ \\
\hline BHP (1)producer & $250 \mathrm{psi}$ \\
\hline Initial pressure & $1470 \mathrm{psi}$ \\
\hline Constant injection rate & $160 \mathrm{STB} / \mathrm{day}$ \\
\hline Sor & 0.2 d.less \\
\hline Swc & 0.35 d.less \\
\hline
\end{tabular}

Table 2 - Reservoir properties which are varied in the sensitivity testing.

Iesting the reservoir model

Sensitivity analyis

\begin{tabular}{lr}
\hline Grid Refinement & $15 \times 10 \times 1$ \\
$15 \times 10 \times 2$ \\
$15 \times 10 \times 2$ \\
$150 \times 10 \times 5$ \\
$150 \times 10 \times 10$ \\
$150 \times 30 \times 5$ \\
$150 \times 50 \times 5$ \\
Timestep refinement & 1 day \\
& 0.1 day \\
Mobility Ratio & 0.01 day \\
& 1 \\
& $10(\mu 0=10 \mathrm{cp})$
\end{tabular}


Table 3 - Reservoir geometry and parameters used in spectral analysis.

Reservoir Simulation Model

Only water production

\begin{tabular}{|c|c|}
\hline Reservoir dimensions & $1500 \times 150 \times 20 \mathrm{ft} 3$ \\
\hline Grid refinement & $150 \times 50 \times 5$ \\
\hline Gridblock dimensions & $10 \times 3 \times 4$ ft3 \\
\hline Interwell distance & $1508 \mathrm{ft}$ \\
\hline Production phase & water \\
\hline$\phi$ & 0.1 d.less \\
\hline \multirow[t]{2}{*}{$\mathbf{k}$} & 100 md \\
\hline & $1000 \mathrm{md}$ \\
\hline$\mu w$ & 1 cp \\
\hline Bw & $1.01 \mathrm{RB} / \mathrm{STB}$ \\
\hline \multirow[t]{5}{*}{ Cw } & 1.00E-04 1/psi \\
\hline & $2.00 \mathrm{E}-05 \mathrm{1} / \mathrm{psi}$ \\
\hline & $1.00 \mathrm{E}-05 \mathrm{1} / \mathrm{psi}$ \\
\hline & $5.00 \mathrm{E}-06 \mathrm{1} / \mathrm{psi}$ \\
\hline & 1.00E-06 1/psi \\
\hline BHP @producer & $250 \mathrm{psi}$ \\
\hline Initial pressure & $1470 \mathrm{psi}$ \\
\hline Duration & 4096 days \\
\hline
\end{tabular}

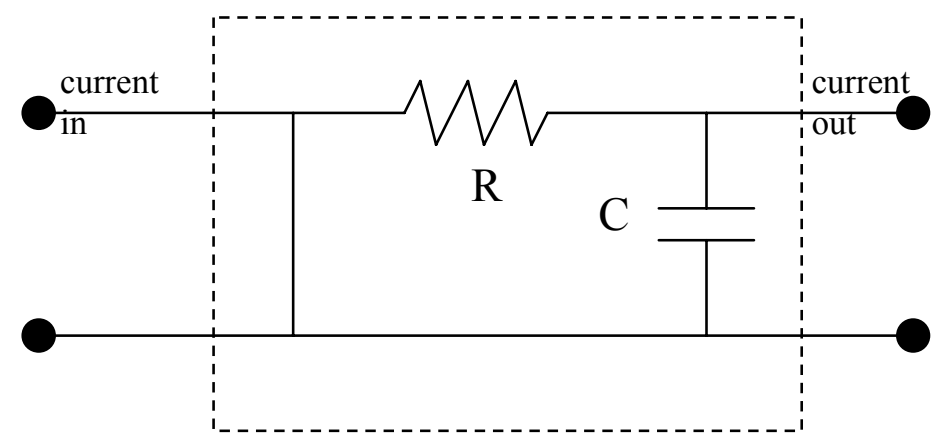

Fig. 1 - A 1-D electrical model with elements for resistance (R) and capacitance (C).
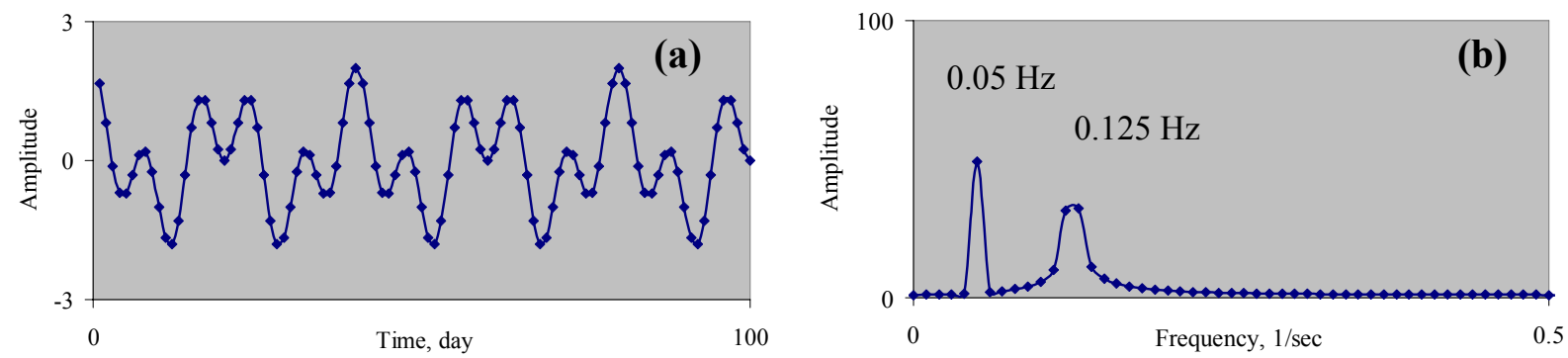

Fig. 2 - Representation of a given sinusoid in time domain(a) and in frequency domain(b). 


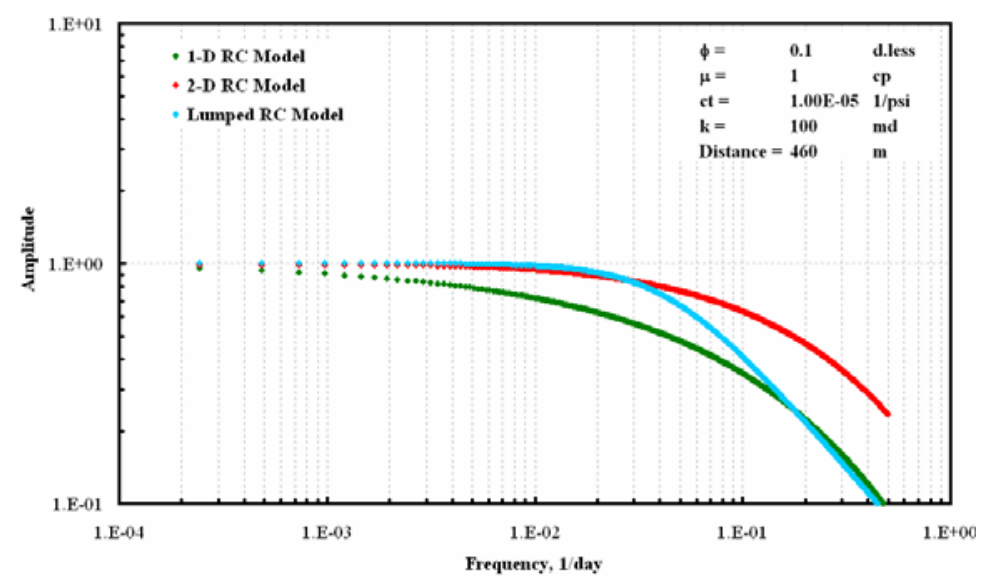

Fig. 3 - Representation of RC network models.

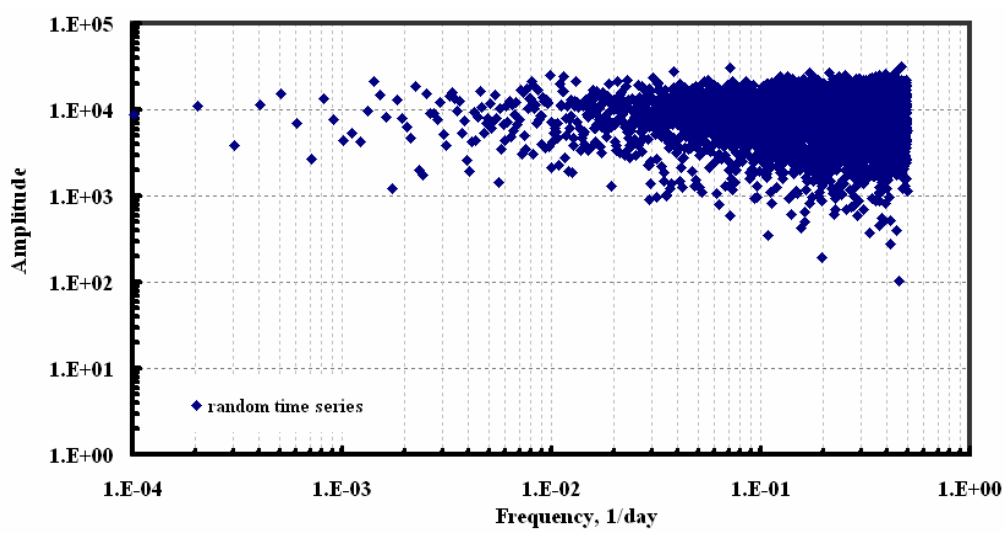

Fig. 4 - Fourier spectrum of 10,000 point random time series.
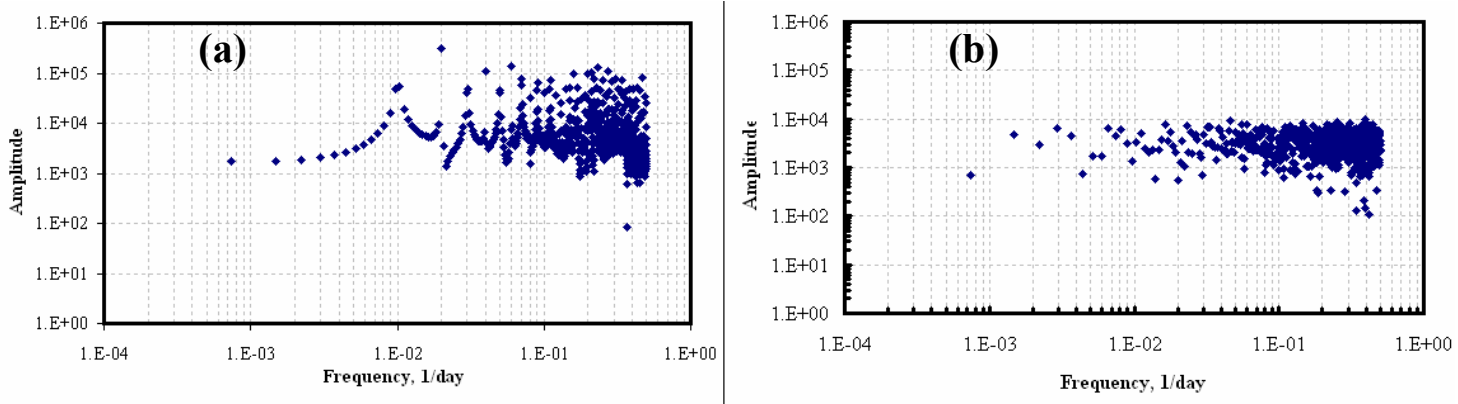

Fig. 5 - Fourier spectra of 1500 point time series of cyclic data(a) and random data(b). 


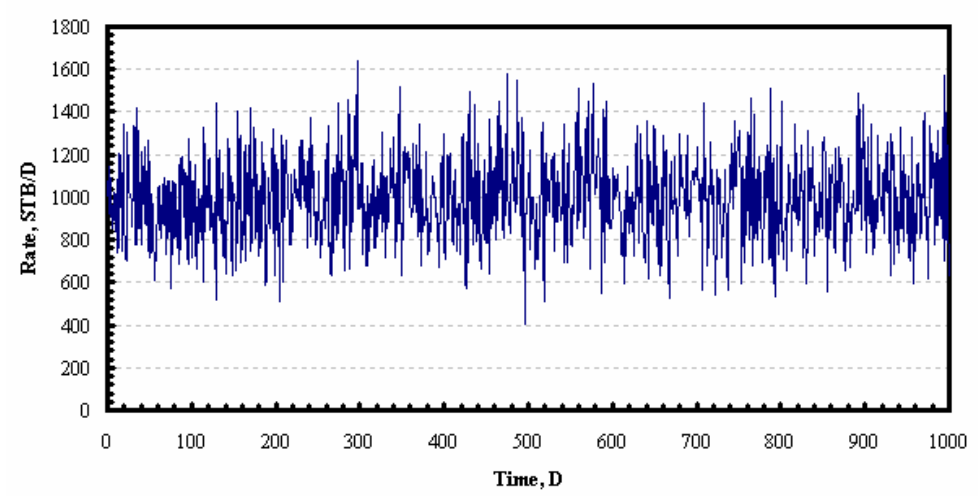

Fig. 6 - Daily injection rate used in reservoir simulation.

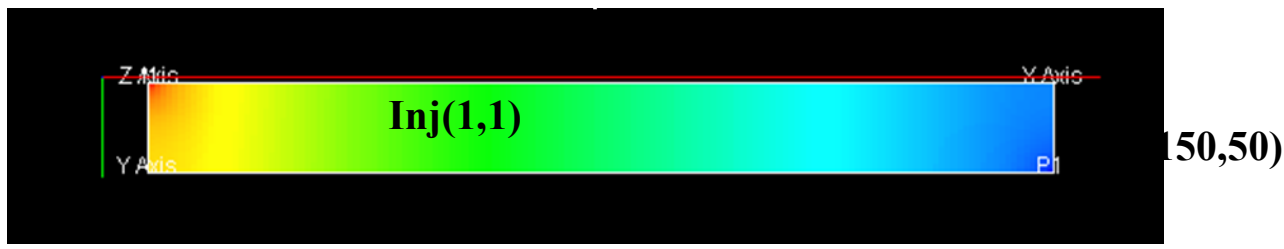

Fig. 7 - Reference reservoir geometry.

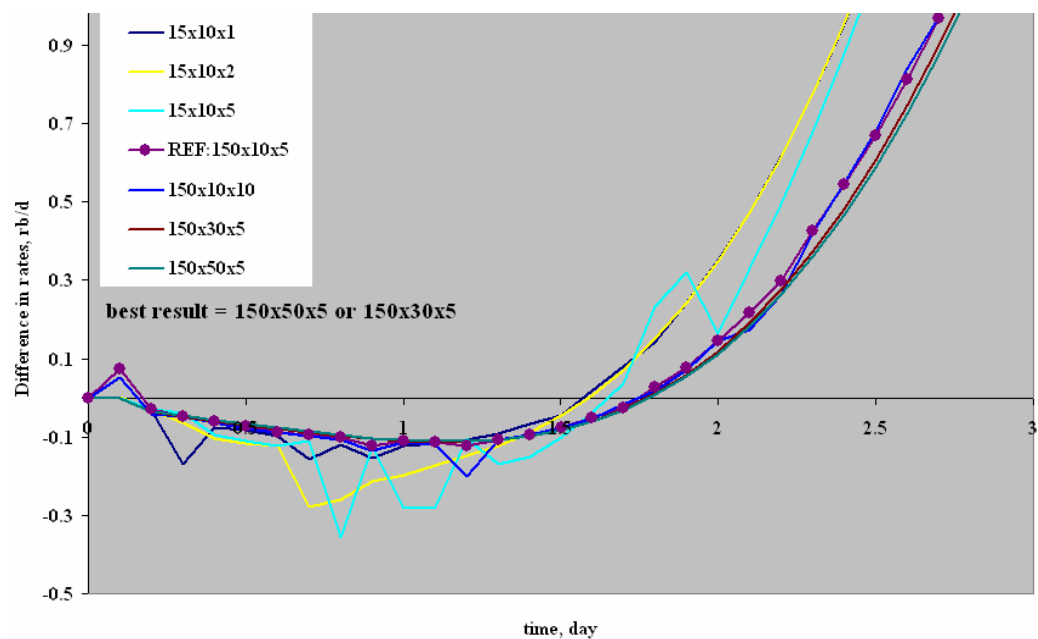

Fig. 8 - Difference between Qin and Qoff at unfavorable mobility ratio( $M=10)$, tstep=0.1 day, Co,w=1e-5 1/psi. 


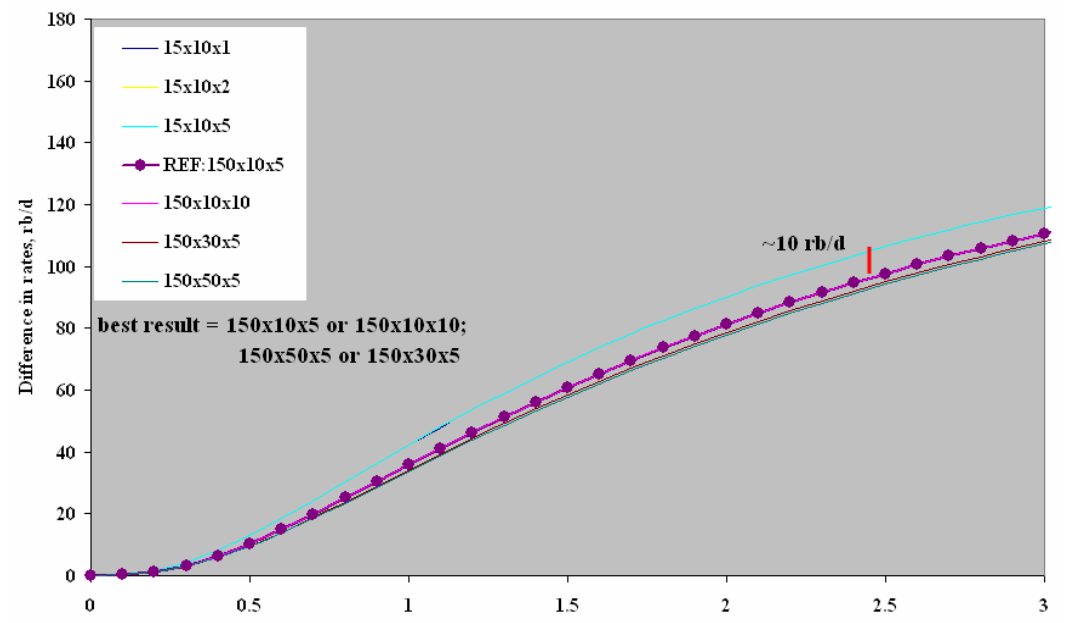

Fig. 9 - Difference between Qin and Qoff at favorable mobility ratio( $M=1)$, tstep $=0.1$ day, Co,w=1e-51/ psi.
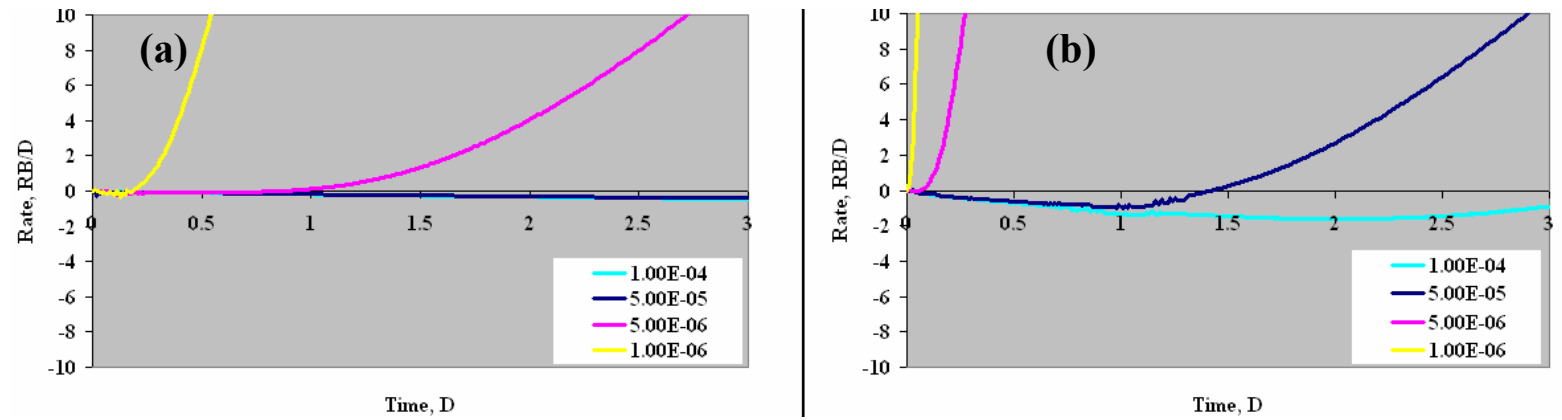

Fig. 10 - Differences between Qin and Qoff at unfavorable mobility ratio, $M=10(a)$ and at favorable mobility ratio, $M=1(b)$ at very fine tstep(0.01 day) and

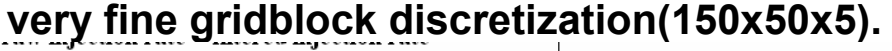
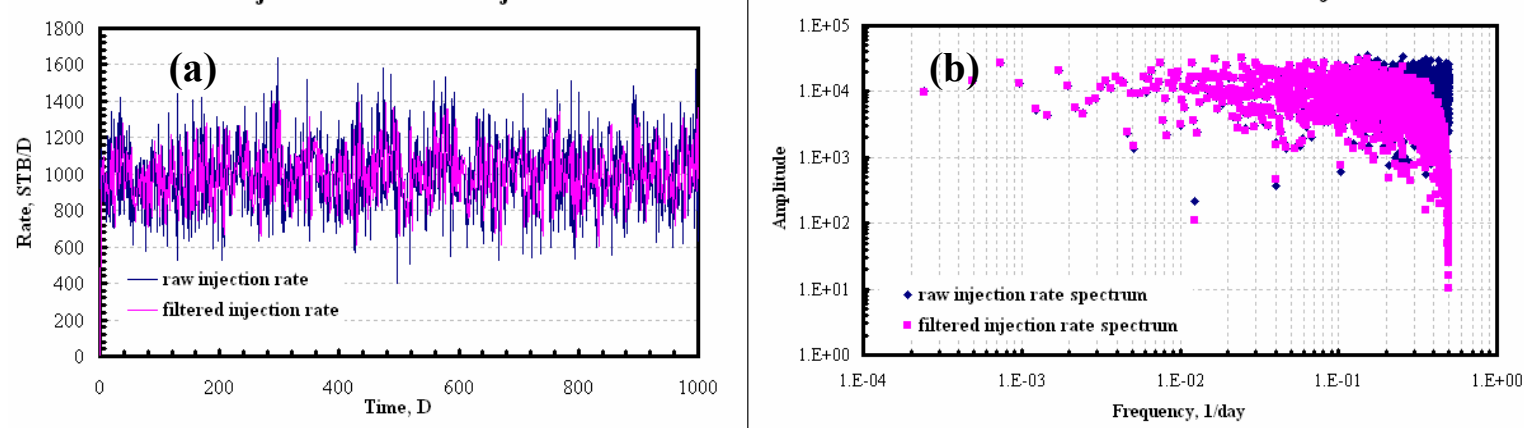

Fig. 11 - Raw and filtered injection rate profile in time domain(a), and in frequency domain(b). 


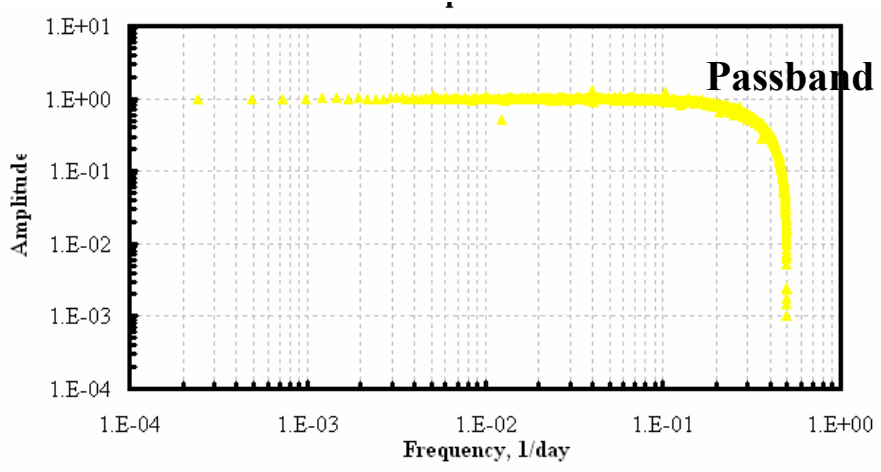

Transition

Attenuation

Fig. 12 - A low-pass filter produced by 4 point - Blackman window.

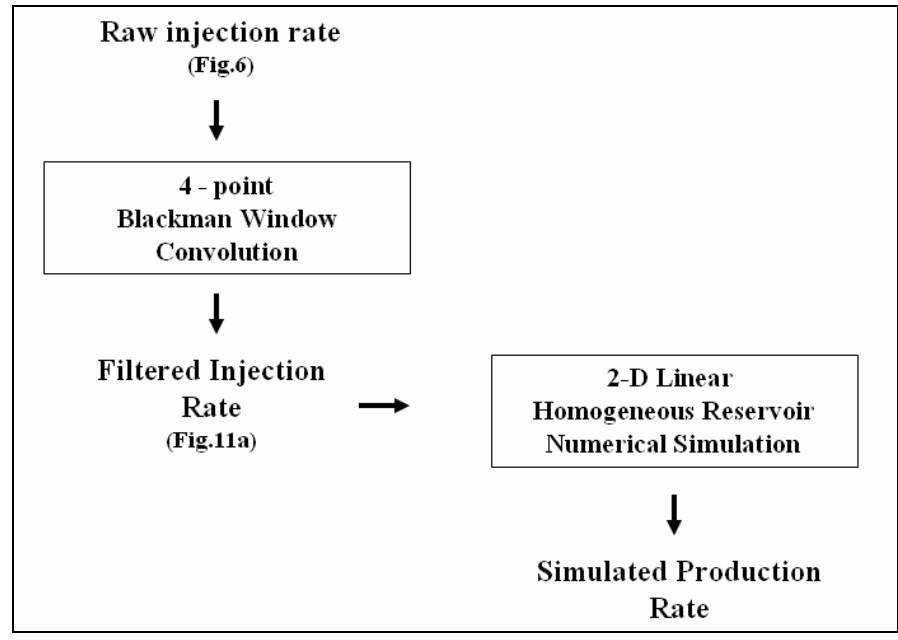

Fig. 13 - Schematic of the filtering procedure.
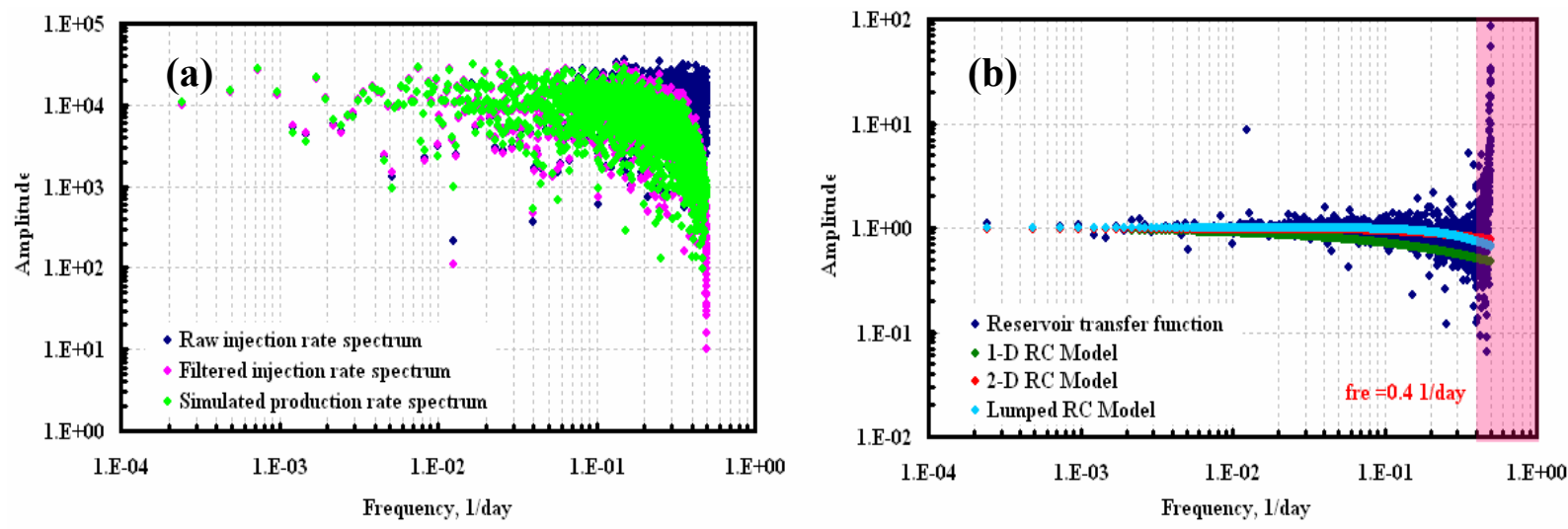

Fig. 14 - Spectral analysis results for $C_{w=1 e-6} 1 / p s i$. (a) Spectra of inj and prod data; (b) Reservoir transfer function compared to three RC models. 

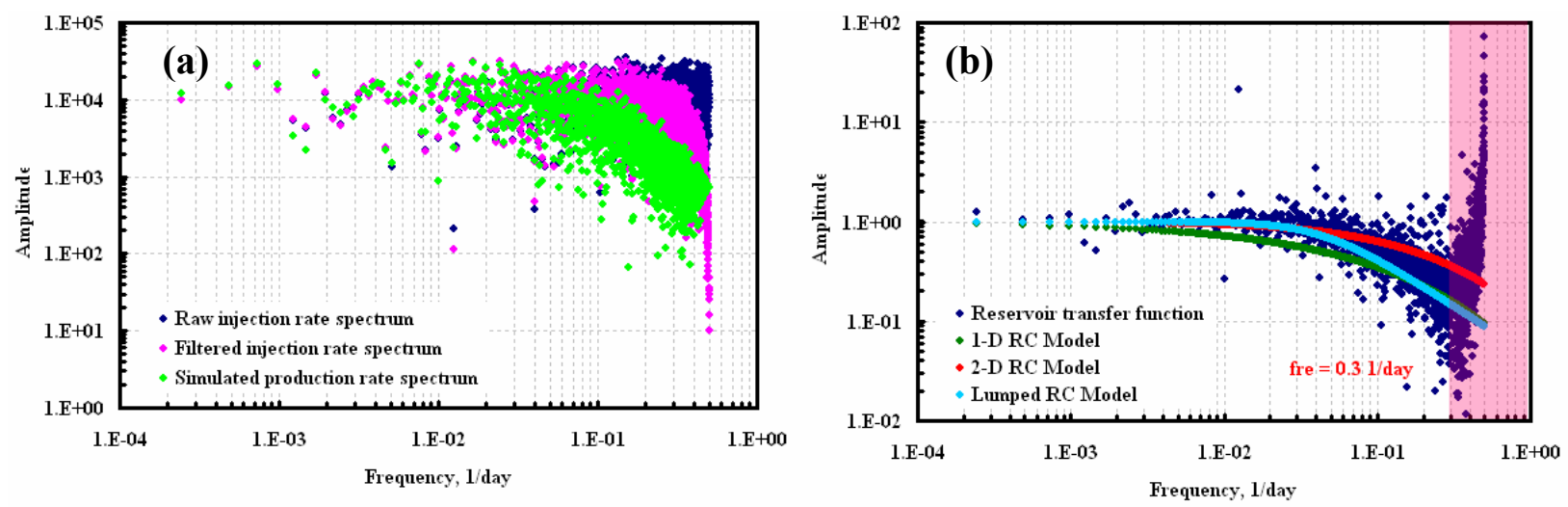

Fig. 15 - Spectral analysis results for $\mathrm{Cw}_{w}=1 \mathrm{e}-51 / \mathrm{psi}$. (a) Spectra of inj and prod data; (b) Reservoir transfer function compared to three RC models.
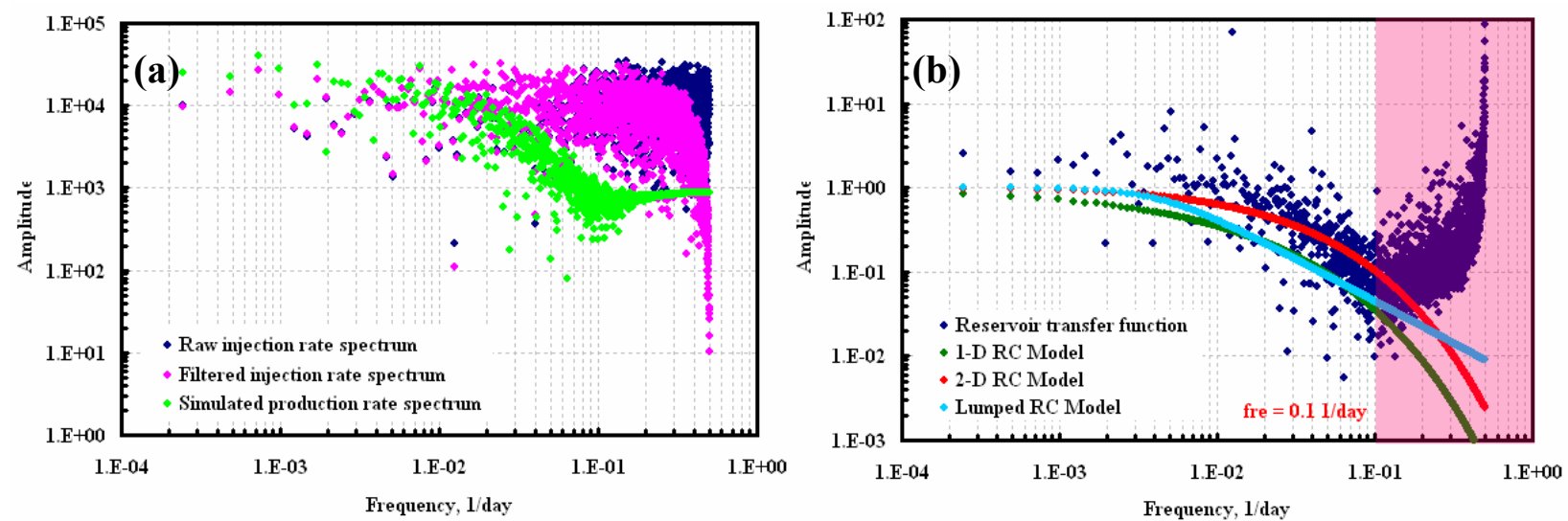

Fig. 16 - Spectral analysis results for $\mathrm{Cw}_{w}=1 \mathrm{e}-4$ 1/psi. (a) Spectra of inj and prod data; (b) Reservoir transfer function compared to three RC models.

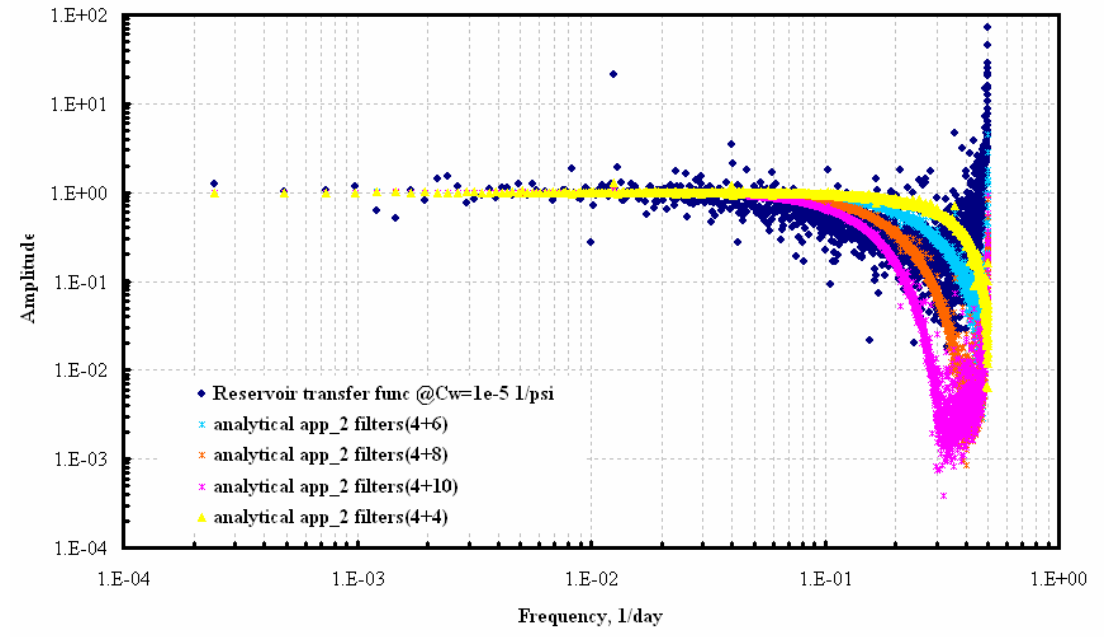

Fig. 17 - Analytical solution to the problem occurring at high frequencies. 


\section{RESULTS AND DISCUSSION \\ PART 4. FURTHER INVESTIGATION OF CAPACITANCE MODEL CHARACTERISTICS}

\subsection{Introduction}

The capacitance model (CM) has been applied to several field and simulated cases to test its abilities. While it has shown promising results, there remain several important problems with the method. Here, we list those problems and some possible methods to overcome them.

\subsection{Negative Weights}

Several field cases using the CM gave rise to negative (or greater than one) interwell connectivity coefficients $(\lambda)$ which are physically meaningless. However, for the simulation cases, which had sufficient data and closed boundaries, this problem was not observed. To prevent meaningless values, we can add this constraint $(0 \leq \lambda \leq 1)$ as one of the constraints during the optimization process. But applying this constraint can still pose problems, including failure to converge. To overcome this problem, we have changed the CM solution procedure so that the $\lambda$ 's are no longer determined using regression formula, but instead they are found in an iterative procedure similar to that used for the $\tau$ 's. In other words, for each initialization, we initialize both the $\tau$ 's and $\lambda$ 's and the optimization procedure tries to decrease the error of the predicted production. Using this method, we do not have the problems of failing convergence and violating the constraints. However, it decreases the $\mathrm{R}^{2}$ of the predictions. For example, for one case using the CM several negative weights were obtained, while using this procedure we do not have the negative weights problem (Table 1), but smaller $\mathrm{R}^{2}$. To get the best answer using this method, we need to resolve the regression equation considering the $\lambda$ constraints through a Lagrange multiplier and use that in the CM.

\subsection{Estimation of confidence interval and maximum permissible value of the estimated $\tau$}

In several cases, we may find a set of $\tau$ 's that are less than the injection-production data sample period $\Delta \mathrm{t}$ (e.g. 30 days or 1 month). In other words, the $\tau$ values are smaller than the resolution of the data. Also, in some cases, we may find very large values for $\tau$. Using these values for $\lambda$ estimation creates no problem, but using them to produce $\tau-\lambda$ plots (Yousef et al., 2006) may lead to incorrect interpretations. Thus, finding confidence intervals and maximum permissible values for $\tau$ is needed.

For estimation of the confidence interval, it may appear that $\tau$ estimates must be integer multiples of $\Delta t$. For example, if we take samples every month:

$q_{1}\left(t_{m}\right)=q(m \Delta t)$

where $q_{1}\left(t_{m}\right)$ is the assigned production rate to m-th time point (Fig. 1), we can have only the information of the sampling day and not the changes during a month. So, finding $\tau$ based on these samples, other than integer multiple of $\Delta \mathrm{t}$ is meaningless. But, monthly production data are samples of data after applying a thirty-day moving average filter on them:

$$
q_{2}\left(t_{m}\right)=\frac{1}{30} \sum_{i=1}^{30} q(i)
$$


where $q_{2}\left(t_{m}\right)$ is the monthly production data (Fig. 1). So they have some information about whole the month and not only the sampling day. To check the ability of monthly data to carry daily information, a very simple case was tried. In this case one injector and one producer were used. Two different daily injection rates were used (Fig. 1) and, using the CM with different $\tau$ 's, the expected production rates were estimated for each $\tau_{\mathrm{d}}$. By integration the daily data, the monthly injection and production data were calculated. Then the $\tau$ associated with the lowest variance of error on monthly data $\left(\tau_{\mathrm{m}}\right)$ for each $\tau_{\mathrm{d}}$ was found. Based on this study, the true $\tau_{\mathrm{d}}$ could be estimated using monthly data by some corrections, even for values less than a month. (Fig.2) Although, for this simple case using monthly data could give us an estimate of $\tau$ for daily data, checking more cases with more wells and also noisy data is needed.

To find the maximum value of $\tau$, we can start with a one injector-one producer model. In this case, different values of $\tau$ are used in the CM with known injection rate $i(t), t=0,1, \ldots t_{\max }$ to calculate the expected production rates. The maximum possible value of $\tau$ is when there is no significant change on the predicted production over the duration of the study, $t_{\max }$. This procedure should be checked also with larger number of wells and using the results we can decide about the maximum possible $\tau$.

\subsection{Short period of data}

In the CM, if we have $n_{i}$ injectors, for each production well we have $2 n_{i}+3$ unknown parameters: $n_{i}+1$ values of $\tau$ and $n_{i}+2$ values of $\lambda$. So we need at least $2 n_{i}+4$ months of data where none of the assumptions, as listed in Yousef et al. (2005), of the model are violated. However, to obtain a more reliable answer, we need more data. Looking at the production history of a field, it may be difficult to find such a period where all assumptions are satisfied. For example, in the production history of a field, we can find some workovers on some wells or drilling new wells each year. To overcome this problem, one possible approach is to apply the model on smaller periods, and fewer wells. In this approach, the model will be applied from very early to last days of injectionproduction data and the coefficients will be found for each well during the history of the field including or not-including the other production wells. In other words, the coefficients are compared after adding each well. Using this approach, the information from whole the waterflooding history (and not only the period where the number of the wells was constant) will be extracted.

\subsection{Conclusions}

The CM has several limitations which would benefit from further attention. These include:

- Obtaining negative weights during optimization procedure in field data. Redefining the approach to find $\lambda$ 's in an iterative procedure can help us to over come this problem. Furthermore, using Lagrange multiplier to modify the regression equation may be useful.

- Confidence interval and maximum permissible value of $\tau$. Obtaining very small or very large values of $\tau$ and using them to produce $\tau-\lambda$ plots may lead to incorrect interpretations. So finding the confidence interval of $\tau$ 's and also maximum permissible value of $\tau$ is needed.

- Short period of proper data. In field data, finding a period with enough data points where all assumptions of CM are satisfied may be difficult. Applying the model within different time periods and comparing the coefficients may help us to obtain much more information out of the data. 


\subsection{Tables and Figures}

Table 1: The calculated coefficients using $C M$ and modified $C M$. Using the modified CM, we have no negative weights but $R^{2}$ has decreased from $97.1 \%$ to $75.5 \%$.

\begin{tabular}{|c|c|c|}
\hline CM & $\begin{array}{c}\text { Modified } \\
\text { CM }\end{array}$ \\
\hline$\tau_{1}$ & 24.57 & 69.80 \\
\hline$\tau_{2}$ & 9.25 & 78.28 \\
\hline$\tau_{3}$ & 1.00 & 117.35 \\
\hline$\tau_{4}$ & 448.97 & 56.07 \\
\hline$\tau_{5}$ & 59.78 & 38.32 \\
\hline$\tau_{6}$ & 98.88 & 99.42 \\
\hline$\tau_{7}$ & 1.00 & 92.25 \\
\hline$\tau_{8}$ & 1.00 & 0.00 \\
\hline$\tau_{9}$ & 1.00 & 88.10 \\
\hline$\tau_{10}$ & 1.00 & 117.02 \\
\hline$\tau_{11}$ & 90.99 & 135.55 \\
\hline$\tau_{12}$ & 260.77 & 19.63 \\
\hline$\tau_{13}$ & 101.07 & 70.16 \\
\hline$\tau_{14}$ & 35.43 & 6.20 \\
\hline$\tau_{15}$ & 1.00 & 43.38 \\
\hline$\tau_{16}$ & 1.00 & 73.37 \\
\hline$\tau_{17}$ & 210.41 & 230.35 \\
\hline$\tau_{p}$ & 28.71 & 78.89 \\
\hline
\end{tabular}

\begin{tabular}{|c|c|c|}
\hline & CM & $\begin{array}{c}\text { Modified } \\
\text { CM }\end{array}$ \\
\hline$\lambda_{1}$ & -0.1036 & 0.000001 \\
\hline$\lambda_{2}$ & -0.1248 & 0.000001 \\
\hline$\lambda_{3}$ & -0.0009 & 0.000001 \\
\hline$\lambda_{4}$ & -0.0840 & 0.000001 \\
\hline$\lambda_{5}$ & 0.4950 & 0.1279 \\
\hline$\lambda_{6}$ & -0.6376 & 0.000001 \\
\hline$\lambda_{7}$ & 0.0017 & 0.000001 \\
\hline$\lambda_{8}$ & 0.0017 & 0.0261 \\
\hline$\lambda_{9}$ & -0.0045 & 0.000001 \\
\hline$\lambda_{10}$ & 0.0048 & 0.000001 \\
\hline$\lambda_{11}$ & -0.2118 & 0.000001 \\
\hline$\lambda_{12}$ & 0.9489 & 0.1977 \\
\hline$\lambda_{13}$ & 0.3522 & 0.000001 \\
\hline$\lambda_{14}$ & -0.1832 & 0.000001 \\
\hline$\lambda_{15}$ & 0.0060 & 0.000001 \\
\hline$\lambda_{16}$ & -0.0008 & 0.000001 \\
\hline$\lambda_{17}$ & -0.1222 & 0.000001 \\
\hline$\lambda_{\mathrm{p}}$ & -1.0588 & 1.0000 \\
\hline & &
\end{tabular}




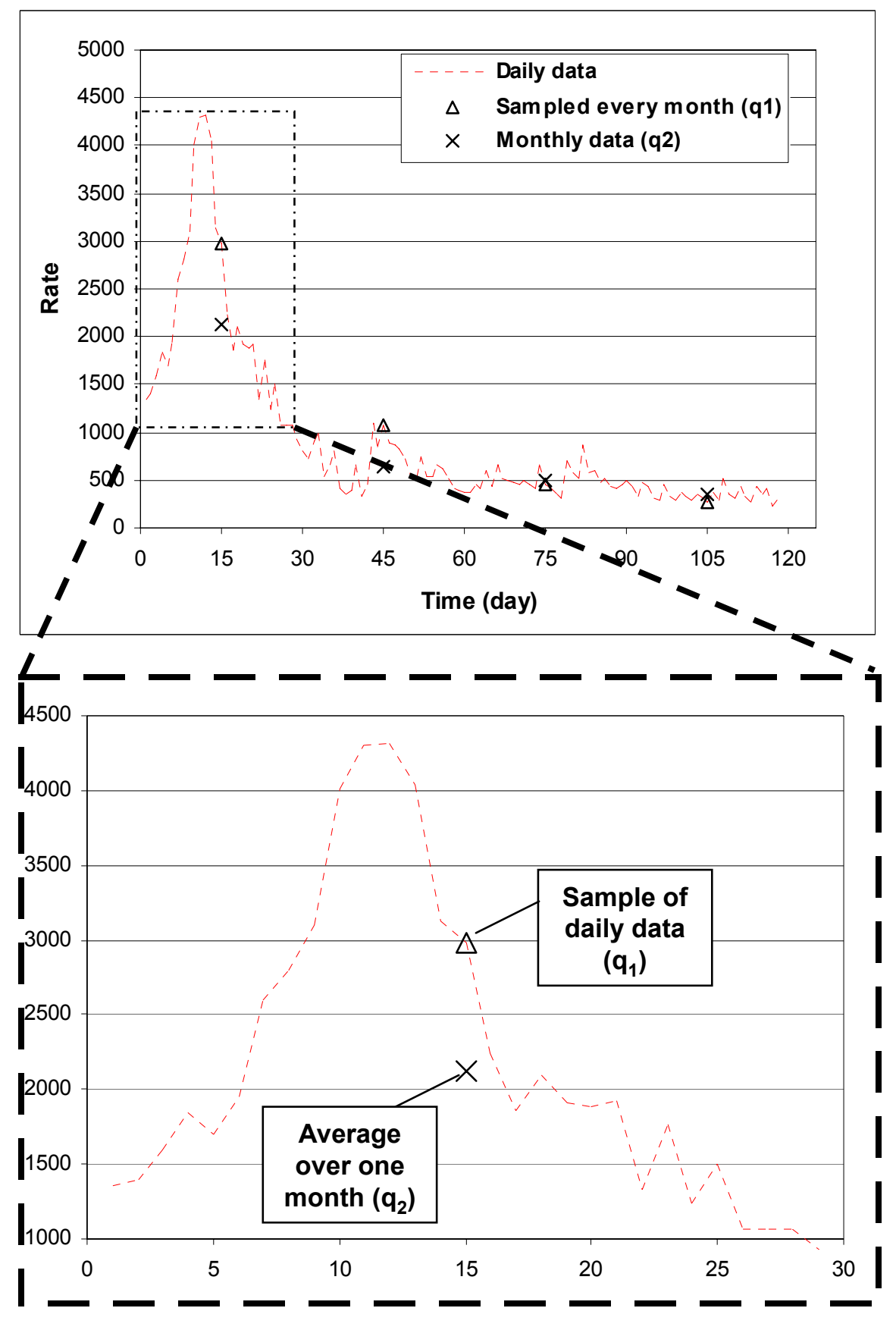

Figure 1: Monthly production data $\left(q_{2}\right)$ and samples from daily data $\left(q_{1}\right)$ 

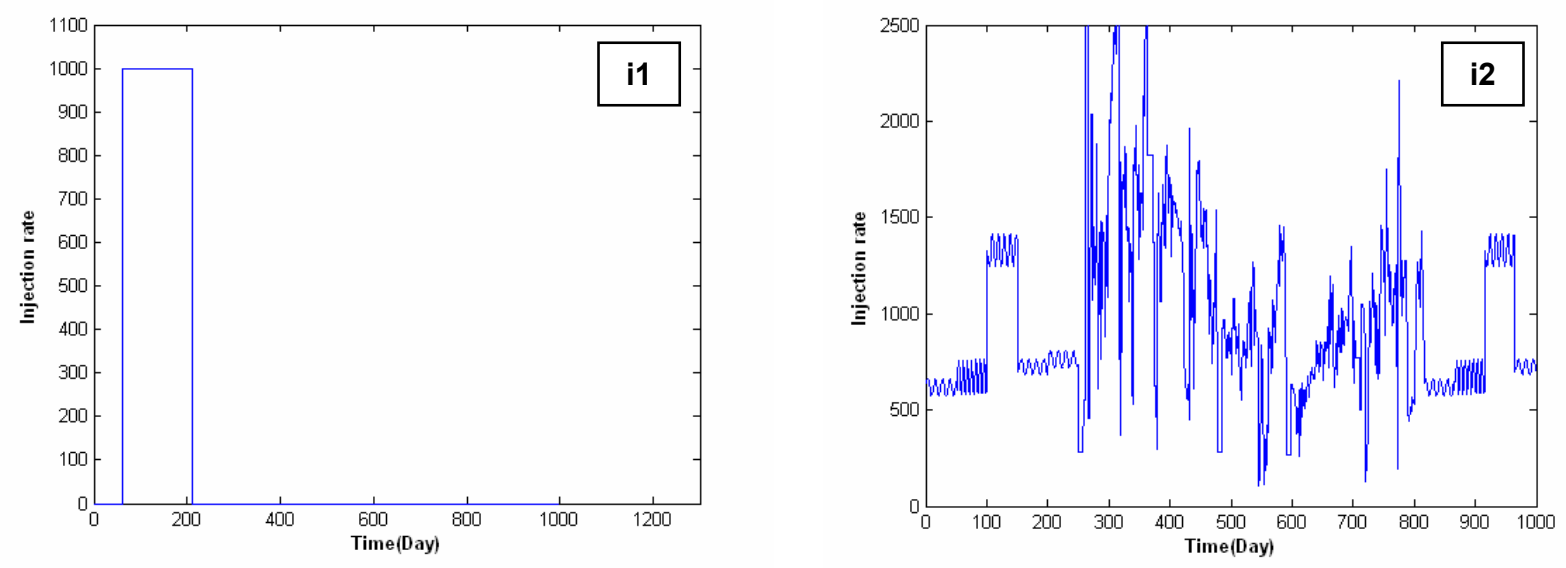

Figure 2: The two different daily injection rates used to find the minimum $\mathbf{T}$

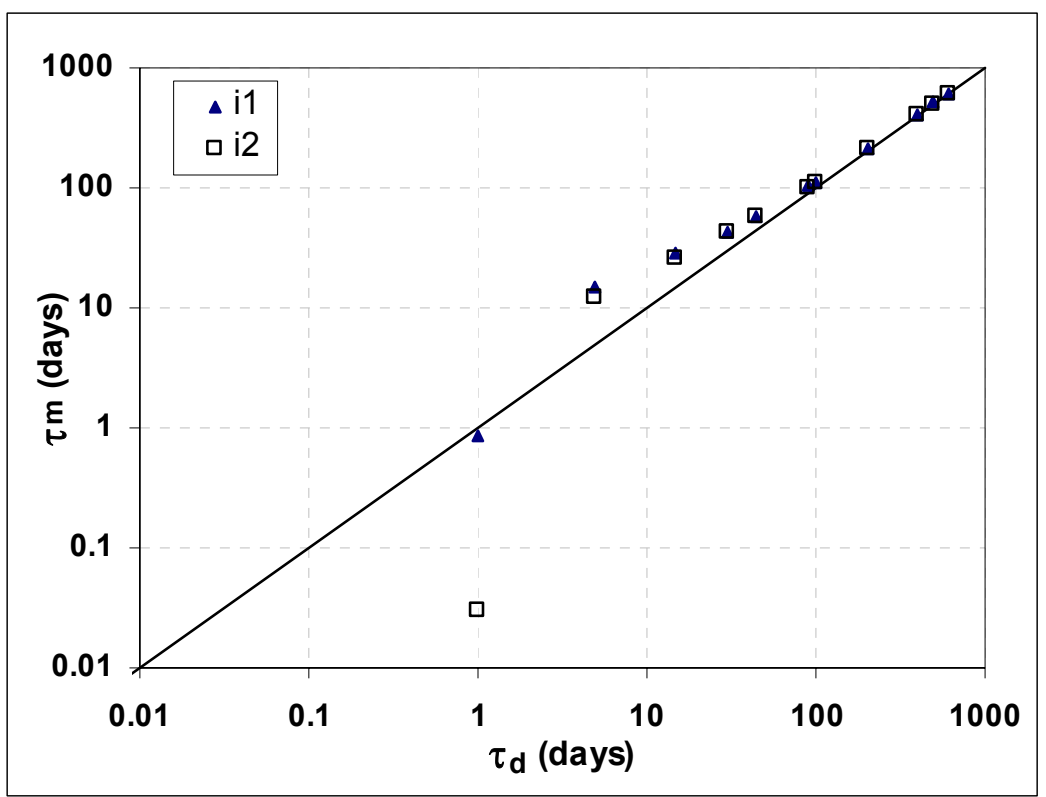

Figure 3: Estimation of $\mathrm{T}$ using monthly data versus the real $\mathrm{T}$ based on daily data. It can be seen that the real $\mathrm{T}$ could be estimated using monthly data even for $\mathrm{T}$ less than a month. 


\section{CONCLUSIONS}

- The capacitance model $\tau$ 's and $\lambda$ 's can be combined to enhance inference about preferential transmissibility trends and the presence of flow barriers.

o Two different methods to combine the $\tau$ 's and $\lambda$ 's have been developed: one representation is the log-log plot of the $\lambda$ 's against the $\tau$ 's for a producer and nearby injectors; another representation is the flow capacity plot where the $\lambda$ 's and the $\tau$ 's are combined using the idea of Lorenz plots.

o The synthetic field applications show that the relation between the $\lambda$ 's and the corresponding $\tau$ 's are consistent with known heterogeneity, the distance between wells, and their relative positions. The flow capacity plots and the log-log plots are capable of identifying whether the connectivity of an injector-producer well pair is through fractures, a high-permeability layer, or through partially completed wells.

o Application of the methods to the South Wasson Clear Fork Field suggest fractures are present.

o Application to the North Buck Draw Field suggests that there is considerable variability in the communicating pathways between injectors and producers.

- An optimization procedure using a simplified capacitance model has been developed and tested on simulated data. Using various objective functions for future oil production, application of the method showed that

0 the proposed capacitance model can successfully capture the true attenuation and time lag behavior between injectors and producers.

0 the formulation of optimal oil production can be used to maximize the future economic return of the asset and is appropriate for simultaneous optimization of well rates in the field where wells are shut-in frequently.

o the proposed optimization method can be used for real-time production control because all of the variables required for the model can be measured and the discrete data can be directly incorporated in the formulation.

- Using spectral analysis, the injection-production interaction can be viewed as a filter. Analysis of simulated injection and production data showed

o The filter model that best fit the reservoir transfer functions is the lumped RC model.

o Changing reservoir model dimensions still showed the lumped RC model to give the best correspondence to the filtering effect of the reservoir.

- The capacitance model has several limitations which would benefit from further attention, including

o Obtaining negative weights during the analysis of field data. Redefining the approach to find the $\lambda$ 's in an iterative procedure can help us to overcome this problem. Furthermore, using a Lagrange multiplier to modify the regression equation may be useful.

o Confidence intervals and maximum permissible values of the calculated $\tau$ 's. Obtaining very small or very large values of $\tau$ and using them to produce $\tau-\lambda$ plots 
may lead to incorrect interpretations. Finding the confidence interval of $\tau$ 's and also maximum permissible value of $\tau$ is needed.

o Short duration situations. In field data, finding a time interval with enough data points where all assumptions of the capacitance model are satisfied may be difficult. Applying the model within different time periods and comparing the coefficients may help us to obtain more information from the data. 


\section{REFERENCES}

Albertoni, A. and Lake, L.W., 2003. Inferring connectivity only from well-rate fluctuations in waterfloods, SPE Reservoir Evaluation and Engineering Journal, 6, 6-16.

Barnes, D.A., Humphrey, K. and Mullenberg, L., 1990. A production optimization system for western Prudhoe Bay Field, Alaska, SPE 20653 presented at the $65^{\text {th }}$ Annual Technical Conference and Exhibition held in New Orleans, Louisiana, U.S.A., 23-26 September.

Buitrago, S., Rodriguez, E. and Espin, D., 1996. Global optimization techniques in gas allocation for continuous flow gas lift systems, SPE 35616 presented at the Gas Technology Symposium held in Calgary, Alberta, Canada, 28 April-1 May.

Burbank, D., "Early $\mathrm{CO}_{2}$ Flood Experience at the South Wasson Clearfork Unit," SPE 24160, presented at the SPE/DOE Eighth Symposium on Enhanced Oil Recovery, Tulsa, Oklahoma, 22-24 April, 1992.

Cortez, O. D., and Corbett, P. M., "Time-Laps Production Logging and the Concept of Flowing Units," SPE 94436, presented at the SPE Europec/EAGE Annual Conference held in Madrid, Spain, June 13-16, 2005.

Craig, F. F., The Reservoir Aspects of Waterflooding, SPE Monograph Series, SPE, Richardson, Texas, 1971.

De Sant Anna'Pizzaro, J.O.: "Estimating injectivity and lateral autocorrelation in heterogeneous media ," PhD dissertation, U. of Texas at Austin, Austin, Texas (1998).

Dutta-Roy K. and Kattapuram, J., 1997. A new approach to gas-lift allocation optimization, SPE 38333 presented at the SPE Western Regional Meeting held in Long Beach, California, U.S.A., 25-27 June.

Edgar, T.F., Himmelblan, D.M. and Lasdon, L.S., 2001. Optimization of chemical processes, second edition, McGraw-Hill Co. Inc., New York.

Everitt, W.L., and Anner, G. E.: Communication Engineering, third edition, McGraw Hill Inc., U.S.A. (1956).

Fang, W.Y. and Lo, K.K., 1996. A generalized well-management scheme for reservoir simulation. SPE Reservoir Engineering, 11(2), 116-120.

Gentil, P.H., 2005. The use of multilinear regression models in patterned waterfloods: physical meaning of the regression coefficients, M.S. Thesis, The University of Texas at Austin.

Gunter, G. W., Finneran, J. M., Hartmann, D. J., and Miller, J. D., "Early Determination of Reservoir Flow Units Using an Integrated Petrophysical Method," SPE 38748, presented at the SPE Annual Technical Conference and Exhibition held in San Antonio, Texas, October 58, 1997.

Harman, T.L. et.al.: Advanced Engineering Mathematics with Matlab, Brooks/Cole Inc., Pacific Grove, California (2000).

Heffer, K.J., Fox, R.J., and McGill, C.A.: "Novel Techniques Show Links Between Reservoir Flow Directionality, Earth Stress, Fault Structure and Geomechanical Changes in Mature Waterfloods," paper SPE 30711 presented at the 1995 SPE Annual Technical Conference and Exhibition, Dallas, 22-25 October.

Hollaender F. et.al: "Harmonic Testing for Continuous Well and Reservoir Monitoring," paper SPE 77692 presented at the 2002 SPE Annual Technical Conference and Exhibition, San Antonio, Texas, 29 September -12 October.

Ibragimov, A., Khalmanova, D., Valko, P., and Walton, J., "On a Mathematical Model of the Productivity Index of a Well from Reservoir Engineering," Journal of Applied Mathematics, Vol. 65, pp. 1952-1980, 2005. 
Jansen, F.E. and Kelkar, M.G.: "Application of Wavelets to Production Data in Describing InterWell Relationships," paper SPE 38876 presented at the 1997 SPE Annual Technical Conference and Exhibition, San Antonio, Texas, 5-8 October.

Jensen, J.L., Lake, L.W., Yousef, A.A., Gentil, P. and Demiroren, N.: "Interwell Connectivity and Diagnosis Using Correlation of Production and Injection Rate Data in Hydrocarbon Production," annual report, Contract No. DE-FC26-03NT15397, U.S. DOE, Washington, DC (August 2005).

Johnson, C.R., et.al.: "Pulse-Testing: A New Method for Describing Reservoir Flow Properties Between Wells," paper SPE 1517 presented at 1966 SPE $41^{\text {st }}$ Annual Fall Meeting, Dallas, Texas, 2-5 October.

Kosmidis, V.D., Perkins, J.D. and Pistikopoulos, E.N., 2005. A mixed integer optimization formulation for the well scheduling problem on petroleum fields, Computers and Chemical Engineering, 29, 1523-1541.

Kosmidis, V.D., Perkins, J.D., and Pistikopoulos, E.N., 2004. Optimization of well oil rate allocations in petroleum fields, Industrial and Engineering Chemistry Research, 43, 35133527.

Kuo, C.H.: "Determination of Reservoir Properties from Sinusoidal and Multirate Flow Tests in One or More Wells," paper SPE 3265 presented at 1971 SPE $46^{\text {th }}$ Annual Fall Meeting, New Orleans, Louisiana, 3-6 October.

Lake, L. W., Enhanced Oil Recovery, Prentice-Hall, Inc., New York, pp. 195-196, 1989.

Larsen, L., "General Productivity Models for Wells in Homogeneous and Layered Reservoirs," paper SPE 71613 presented at the SPE Annual Technical Conference and Exhibition, New Orleans, Louisiana, September 30-October 3, 2001.

Lee, J. et.al.: Pressure Transient Testing, Textbook Series, SPE, Vol. 9, Richardson, Texas, (2003).

Litvak, M.L., Clark, A.J., Fairchild, J.W., Fossum, M.P., MacDonald C.D. and Wood, A.R.O., 1997. Integration of Prudhoe Bay surface pipeline network and full field reservoir models, SPE 38885 presented at the SPE Annual Technical Conference and Exhibition held in San Antonio, Texas, U.S.A., 5-8 October.

Martinez, E.R., Moreno, W.J., Moreno, J.A. and Maggiolo, R., 1994. Application of genetic algorithm on the distribution of gas lift injection, SPE 26993 presented at the III Latin American/Caribbean Petroleum Engineering Conference held in Buenos Aires, Argentina, 2729 April.

Muskat, M.: Physical Principles of Oil Production, McGraw Hill Inc., U.S.A. (1949).

Panda, M.N. and Chopra, A.K.: "An Integrated Approach to Estimate Well Interactions," paper SPE 39563 presented at the 1998 SPE India Oil and Gas Conference and Exhibition, New Delhi, 17-19 February.

Raghavan, R., Well Test Analysis, Prentice-Hall, Englewood Cliffs, New Jersey, 1991.

Refunjol, B.T., and Lake, L. W., "Reservoir Characterization Based on Tracer Response and Rank Analysis of Production and Injection Rates," paper presented at the Fourth International Reservoir Characterization Technical Conference, Houston, Texas, March 2-4, 1997.

Refunjol, B.T.: "Reservoir characterization of North Buck Draw field based on tracer response and production/injection analysis," MS thesis, U. of Texas at Austin, Austin, Texas (1996).

Rosa, A.J and Horne, R.N.: "Reservoir Description by Well-Test Analysis by Use of Cyclic Flow-Rate Variation," paper SPE 22698 presented at 1991 SPE Annual Technical Conference and Exhibition, Dallas, Texas, 6-9 October. 
Schmalz, J. P., and Rahme, H. S., "The Variation in Water Flood Performance with Variation in Permeability Profile,” Producers Monthly, pp. 9-12, July, 1950.

Sellars, R., and Hawkins, C., "Geology and Stratigraphic Aspects of the Buck Draw Field, Powder River Basin, Wyoming" Wyoming Geological Association $43^{\text {rd }}$ Annual Field Conference Guidebook, pp. 97-110, 1992.

Shook, G. M., "A Simple Fast Method of Estimating Fractured Reservoir Geometry from Tracer Tests," Transactions of the Geothermal Resources Council, Vol. 27, September 2003.

Smith, Steven W.: The Scientist and Engineer's Guide to Digital Signal Processing, second edition, California Technical Publishing, California (1999).

Soeriawinata, T. and Kelkar, M.: "Reservoir Management Using Production Data," paper SPE 52224 presented at the 1999 SPE MidContinent Operation Symposium, Oklahoma City, Oklahoma, 28-31 March.

Stoisits, R.F., Batesole, E.C., Champion J.H. and Park, D.H., 1992. Application of nonlinear adaptive modeling for rigorous representation of production facilities in reservoir simulation, In SPE 24898 presented at the $67^{\text {th }}$ Annual Technical Conference and Exhibition held in Washington, D.C., U.S.A., 4-7 October.

Wang, P., Litvak, M.L. and Aziz, K., 2002a. Optimization of production operations in petroleum fields, SPE 77658 presented at the Annual Technical Conference and Exhibition held in San Antonio, Texas, U.S.A., 29 September-2 October.

Wang, P., Litvak, M.L. and Aziz, K., 2002b. Optimization of production from mature fields, presented at the 17th World Petroleum Congress, Rio de Janeiro, Brazil, September 1-5.

Yousef, A. A., Gentil, P., Jensen, J. L., and Lake, L. W., "A Capacitance Model to Infer Interwell Connectivity from Production and Injection Rate Fluctuations," SPE 95322, presented at the SPE Annual Technical Conference and Exhibition held in Dallas, Texas, October 9-12, 2005.

Yousef, A. A., Lake, L.W. and Jensen J.L.: "Analysis and Interpretation of Interwell Connectivity From Production and Injection Rate Fluctuations Using a Capacitance Model," paper SPE 99998 presented at the 2006 SPE/DOE Symposium on Improved Oil Recovery, Tulsa, Oklahoma, 22-26 April.

Yousef, A.A., 2006. Investigating statistical techniques to infer interwell connectivity from production and injection rate fluctuations, $\mathrm{PhD}$ dissertation, The University of Texas at Austin. 